\title{
Common duct stones : a reappraisal of etiology and surgical management with special emphasis on operative biliary endoscopy
}

Citation for published version (APA):

Reitsma, B. J. (1981). Common duct stones : a reappraisal of etiology and surgical management with special emphasis on operative biliary endoscopy. [Doctoral Thesis, Maastricht University]. Rijksuniversiteit Limburg. https://doi.org/10.26481/dis.19811002br

Document status and date:

Published: 01/01/1981

DOI:

10.26481/dis.19811002br

Document Version:

Publisher's PDF, also known as Version of record

Please check the document version of this publication:

- A submitted manuscript is the version of the article upon submission and before peer-review. There can be important differences between the submitted version and the official published version of record.

People interested in the research are advised to contact the author for the final version of the publication, or visit the DOI to the publisher's website.

- The final author version and the galley proof are versions of the publication after peer review.

- The final published version features the final layout of the paper including the volume, issue and page numbers.

Link to publication

\footnotetext{
General rights rights.

- You may freely distribute the URL identifying the publication in the public portal. please follow below link for the End User Agreement:

www.umlib.nl/taverne-license

Take down policy

If you believe that this document breaches copyright please contact us at:

repository@maastrichtuniversity.nl

providing details and we will investigate your claim.
}

Copyright and moral rights for the publications made accessible in the public portal are retained by the authors and/or other copyright owners and it is a condition of accessing publications that users recognise and abide by the legal requirements associated with these

- Users may download and print one copy of any publication from the public portal for the purpose of private study or research.

- You may not further distribute the material or use it for any profit-making activity or commercial gain

If the publication is distributed under the terms of Article 25fa of the Dutch Copyright Act, indicated by the "Taverne" license above, 
In memory of Clarence $J$. Schein, a most dedicated teacher in biliary surgery. 


\section{Common Duct Stones}

A reappraisal of etiology and surgical management with special emphasis on operative biliary endoscopy.

\section{Academisch Proefschrift}

ter verkrijging van de graad van doctor in de geneeskunde aan de Rijksuniversiteit Limburg te Maastricht op gezag van de rector magnificus

Prof. Dr. W.H.F.W. Wijnen volgens besluit van het College van Dekanen in het openbaar te verdedigen op vrijdag 2 oktober 1981 des namiddags te 16.00 uur in de aula van de universiteit Tongersestraat 53 door

Bertus Johannes Reitsma geboren te "s-Gravenhage 
Promotors: Prof.Dr. J.M. Greep, surgeon

Prof.Dr. P.J. Brombacher, clinical biochemist

Referents: G. Berci, M.D., surgeon, Los Angeles

Dr. M.N. van der Heyde, surgeon, Arnhem

Prof.Dr. H.C. Hemker, biochemist, Maastricht

Dr. G.P. van Berge Henegouwen, internist, Arnhem.

The publication of this thesis has been enabled by the financial support of:

Karl Storz GmbH \& Co, Tuttlingen, West-Germany

Falk Foundation e.V...Freiburg i.Br., West-Germany

Lamenefis Instruments, Utrecht

Klinisch Genootschap Zuid-Limburg. 
to my parents

Ineke

Bas, Simane, Liesbeth and Annemieke. 


\section{Common Duct Stones}

A reappraisal of etiology and surgical management with special emphasis on operative biliary endoscopy

\section{List of contents \\ Part I}

Gallstone disease

Chapter 1

Cholelithiasis: a true gallbladder disease?

Chapter 2

Lithogenic bile production

2.1 Re-evaluation of lithogenecity

2.2 Cholesterol metabolism

2.3 Cholesterol synthesis

2.4 Bile Acid synthesis

2.5 Bile Acid conjugation

2.6 Bile Acid pool size

2.7 Intestinal phase of Bile Acids

2.8 Postcholecystectomy dynamics in BA metabolism

2.9 BA synthesis during obstruction

2.10 Bile formation

2.11 Bile Salt Dependant Canalicular Bile Flow

2.12 Bile Salt Independant Canalicular Bile Flow

2.13 Vagus influence in bile salt secretion

Chapter 3

Associated metabolic and endocrinologic disorders

3.1 Diet and gallstones

3.2 Hyperlipoproteinemia

3.3 Diabetes Mellitus

3.4 Parathyroid hormones

3.5 Sex hormones 
Chapter 4

Associated pathology
4.1 Acalculous cholecystitis
4.2 Cholesterosis of the gallbladder
4.3 Pancreatitis
4.4 Carcinoma of the biliary system

Chapter 5

Medical stone dissolution

\section{Part II}

Surgical considerations in gallstone disease

Chapter 6

Socio-Economic aspects of complications in Biliary Surgery

Chapter 7

\section{Common Duct Stones}

7.1 Introduction

7.2 Etiology

7.3 Pigment Stones

7.4 Primary Common Duct Stones

7.5 Retained Common Duct Stones

Chapter 8

The Common Bile Duct

8.1 Surgical anatomy

8.2 Width of Common Bile Duct

8.3 Histology of Common Bile Duct

8.4 Distal Common Bile Duct

8.5 Pathology

8.9 Physiology of sphincter mechanism 


\section{Chapter 9 \\ Common Bile Duct Exploration}

9.1 Indications

9.2 Operative cholangiography

9.3 Flow and pressure measurements

9.4 Antibiotics

9.5 Instrumentation

9.6 Stone extraction techniques

9.7 Complications and failures

9.8 Primary closure versus T-tube drainage

Chapter 10

Operative biliary endoscopy (Cholangioscopy)

10.1 Historical review

10.2 Instrumentation

10.3 Sterilization

10.4 Indications

10.5 Technique

10.6 Findings

10.7 Tactic of cholangioscopy

10.8 Complications.

\section{Chapter 11}

Non-operative treatment of Retained Stones

11.1 Decision making

11.2 Chemical dissolution

11.3 Stone extraction through T-tube tract

11.4 Endoscopic retrograde sphincterotomy (EPT)

Chapter 12

Operative treatment of Retained Stones

12.1 Re-exploration

12.2 Bilio-enteric bypass 


\section{Part III}

Investigation of surgical treatment and bile composition in choledocholithiasis

\section{Chapter 13}

Results of Common Bile Duct exploration

\subsection{Introduction}

13.2 Patients and Methods

13.3 Results

13.4 Retained Stones

13.5 Complications

13.6 Mortality in Biliary Surgery

13.7 Bypass Surgery

13.8 Operative Manometry

13.9 Operative Biliary Radiology

13.10 Results of Cholangioscopy

13.11 Conclusions

Chapter 14

The composition of hepatic bile in Choledocholithiasis
14.1 Introduction
14.2 Patient selection
14.3 Methodology bile sampling
14.4 Laboratory methods
14.5 Results
14.6 Discussion

\section{Part IV}

Summary and conclusions

References 
Acknowledgements.

This thesis would not have been realized without the enthousiasm and support of many others.

I would like to express my gratitude especially to Prof. Greep who has been very stimulating in his own dynamic way. By your efforts I was able to gain foreign experience on subjects concerning biliary surgery on behalf of this study. You learned me the basic principles in medical writing and critically analysing the surgical literature.

Prof. Brombacher, your help has been most wellcome.

The experience in cooperation on resident affairs in our hospital could be extended to this clinical scilentific investigation. Your brief, sharp and resolute desicion-making and comment almost made a surgical impression on me.

I would like to express my utmost gratitude to you, George Berci, for your dedicated cooperation on every subject of this thesis. Ever since our first acquaintance I became deeply impressed by your driving personality which has quaranteed the proclamation and the ultimate success of the International Biliary Association. I was most fascinated by your cordial friendship and your dedicated help in finishing this thesis.

Dr. Van der Heyde, Dr. Van Berge Henegouwen, Prof. Hemker, you have all read the manuscript critically and I appreciate your comments.

Lynn Bennion, thank you for reviewing the manuscript on gallstone etiology and medical treatment. Our common interest in gallstone pathogenesis has been extended to a warm friendship.

Piet van Velthoven, Hans van Dijk, Andree Stoop and Pieter de Ruiter, in your surgical department my interest in general surgery has been aroused.

Actually my first clinical experiences with cholangioscopy were performed in Alkmaar which served as the basis for this thesis. I am most thankful for your generous support throughout my residency.

Dr. Pinckaers, Munting, Van Houtte, Lens, Van Duin, I want to thank you for the opportunity practising biliary surgery during my residency.

I also want to thank the hospital administration and all personal of the De Wever Hospital for their actual help, especially the clinical laboratory, operation room and surgical wards. The laboratory work has been performed with great precision and I want to thank Piet Verheesen for the time spent on this important item on gallstone etiology.

I wish to thank Riet Vola and Maartje Duisings particularly who have provided secretarial help most efficiently and accurately.

Chris Voskamp, you provided the lay-out and graphical get-up of the thesis in a friendly but highly professional way.

Frans W/inands, you have been of special help to me serving as the interpretor between the medical and commercial Aesculapius. 
The illustrations and clinical materials were supplied by Laméris Instruments.

The excellent facilities of the medical library of the De Wever Hospital, personally quaranteed by Miss Dessers made it much easier in reviewing the surgical literature. Finally I wish to thank all of my family for their joyful and inspiring friendship especially Ineke because of her forbearance and unnoticed support. 
Part I

Gallstone disease

4 


\section{Chapter 1}

\section{Cholelithiasis: a true gallbladder disease?}

Cholesterol gallstone disease is one of the most common gastro-intestinal disorders encountered in the western world. Prevalence of the disease is increasing and is somehow related to the type of food consumed. Diet is supposed to be still the most important factor in stone disease $(37,38)$.

No other primary gastro-intestinal or metabolic disorder can be considered etiologically of more importance [361].

The female prevelance of the disease is well established and most pronounced in the reproductive years of the female adult. This high female preponderance in cholelithiasis however has never been convincingly correlated to a significant difference in bille composition in the normal male and female adults. The only physiologic difference between male and female is an increase in total Bile Acid which may enhance gallstone formation although this total Bile Acid Pool Size has been convincingly demonstrated to be rather a secondary than a primary event in cholelithiasis.

The female sex hormones seem to play an important role in cholelithogenesis. Hyperoestrogenaemia especially may promote gallstone formation by reducing Bile Acid secretion which favors a rise in cholesteral concentration in bille. These data are supported by clinical evidence showing an increased risk for gallstones in patients which are or have been pregnant and in woman taking contraceptive medication even on a short term regiment. Moreover progesteron has synergic action inducing delayed gallblader emptying as noted during pregnancy and in the second half time of menstrual cycle. These low flow states may easily facilitate growth of incipient gallstones. But also the intestinal phase of Bile Acids during the progesteron phase is increased resulting in an extended Small Bowel Transit Time which may be of additional etiological importance in cholelithogenesis.

These low flow states in the gallbladder are also encountered in patients following vagotomy, single or in combination with gastric resections, significantly increasing the risk for gallstone disease.

The role of the gallbladder in stone disease is still debated (74). Advances in research on Bile Acid biochemistry and kinetics have contributed to a better understanding of gallstone pathophysiology (chapter 2). Supersaturated bile (cholesteroll seems to be of major importance in stone disease $(63,288)$. Underlying hepatic disturbances resulting in bille supersaturated with cholesterol are defined and are responsible for the production of lithogenic bile. 
Different studies have established lower lithogenic indices in cholecystectomized patients 14191 , at hough contrasting data on postoperative bile composition following cholecystectomy have been published $170,242,346,349)$. The gallbladder itself may thus be suspected influencing hepatic bile lipid synthesis and/or secretion by feedback mechanism(s), resulting in a decrease in BA and/or an increase in the production of cholesterol leading to lithogenic bile. This hypothetical mechanism may thus supposed to be eliminated after cholecystectomy in studies showing a lessened lithogenicity in postcholecystectomy patients.

Hepatic lithogenic bile production as the primary event in gallstone disease has probably been overemphazised. This can be substantiated additionally by the disappointing results of medical stone dissolving therapy by oral administration of Bile Acids namely Urso and Cheno Acid which influence hepatic bile production. A reappraisal of the importance of the gallbladder itself in the genesis of cholesterol cholelithiasis should be warranted. The overwhelming number of symptom free patients after cholecystectomy show at least indirect evidence the gallbladder remains the epicenter of this disease.

Different factors of the gallbladder function may play a role as well. The concentrating activity of the gallbladder seems to differ considerably for each bile lipid component. The presence of a selective absorption process in the gallbladder may enhance stratification of bile initiating stone formation 195,232$)$. This selective process further strengthens the essential role of the gallbladder in cholesterol stone disease.

If cholelithiasis is thus regarded primarily a disease of the gallbladder, biliary stones are a secondary manifestation. The confused issue that biliary stones can be discovered after cholecystectomy in a time span from 3 months to 30 years, has encouraged the hypothesis of reformed biliary stones. Furthermore, a clear distinction between recurrent (= reformed) and retained stones cannot be properly assessed on clinical grounds. This is the main reason why there is no uniform understanding on the precise indication forabilio-enteric bypass procedure in stone disease. Migration of stones in the biliary ducts can no doubt lead to infection in the biliary system. Often the inflammation is not limited to the gallbladder alone. Both liver and pancreas and the entire biliary system as well are often involved (13), especially when cholangitis develops.

In general, it is evident from the literature that the indication for surgery should be broad in view of the natural history of the disease. Early surgical treatment eliminates sequallae that can cause complications which will take their toll in life at a later stage $[267,326]$. Primary operations are likely to carry a lower mortality rate than the nonoperative approach in gallstone disease. Effective treatment of biliary 
disease has in the last decades been constantly improved by intraoperative cholangiography in combination with other refinements in technique and / or instrumentation. The cure-rate of Common Duct Stones (CDS) is primarily determined by the incidence of complications being retained stones and iatrogenic leasions in the biliary system, necessitating reoperation.

Although progress has been acknowledged, the vexing problem of retained stones remained one of the most serious complications in biliary surgery (7). Precise indications for surgicall procedures especially for adjunctive surgical measures are of paramount importance. An originally benign disease like cholelithiasis can be followed by a clinically "malignant" course because of the necessity of repeated explorations which are accompanied by a higher complication and mortality rate 176!.

Prevention of retained and recurrent bile duct calculi is the primary goal of biliary surgery. The majority of these sequelae can be prevented by acceptance of routine operative biliary radiology and biliary endoscopy as well.

The aim of this study is to define the etiology of Common Duct Stones by a clinical investigation and to improve the results of surgery by:

1 : Protocolising the surgical treatment towards a standard procedure for Common Duct Exploration,

2: Including a permanent position for cholangiography and billary endoscopy during operation.

3: Irvestigating hepatic bile biochemically in search for changes in bile lipid composition in patients with common duct stones before and after appropriate surgical treatment.

If a considerable reduction in the total score of retained stones can be accomplished by a complete surgical approach $\left\|\frac{1}{\text { and }} 2\right\|$, and the hepatic bile composition in the postoperative period in the treated patients does not give any substantial evidence for a continued lithogenicity (3), the hypothesis on both reformed and recurrent (cholesterol) biliary stones should be redifined:

All biliary stones in the early and late postoperative phase following cholecystectomy $(+/-$ CDE) should thus be regarded as "ordinary" retained stones unless otherwise proven.

In the first part of this study a critical analysis on all aspects of chetelithiasis is presented focusing on the precise role of both hepatic bile lipid formation and the 
role of the gallbladder in cholelithogenesis. Special attention is paid to BA and cholesterol physiology being the main parameters determining lithogenicity. The different hormonal and endocrinologic mechanism which are claimed to be of additional importance in stone disease are reassessed. Also various disease entities in the biliary system are reviewed determining their relation to gallstone disease. A reappraisal of the medical therapy of gallstone disease is presented. The disappointing results so far do not favor the hypothesis proclaiming hepatic lithogenic bile production as the primary event in gallstone disease. The most succesfull results in medical dissolution therapy will probably be achieved as a prophylaxis in patients at high risk to develop gallstones. The indication as an alternative to surgical therapy is limited to a small number of patients.

In the second part of this study the surgical considerations in gallstone treatment are reviewed.

The surgical anatomy of the biliary system and the etiology of Common Duct Stones are reviewed. Special emphasis is paid to Common Duct Exploration focusing on operative biliary endoscopy (chapter 10).

The strategy in treatment of patients with RS is outlined.

In the third part the results of $51 / 2$ years of surgical treatment of cholelithiasis is presented. Different operative diagnostic modalities are evaluated. In patients who have been treated according to a protocol on CDE including cholangiography. hepatic bile composition is investigated to asses the risk for stone formation postoperatively, which is of interest in planning the biliary operation in patients with Common Duct Stones. 


\section{Chapter 2}

\section{Lithogenic bile production}

\subsection{Re-evaluation of lithogenecity}

Bile contains water, conjugated bilirubin, organic and inorganic ions, small amounts of proteins and three types of lipids: Bile Acids (BA), Phospholipids (PPL) and Cholesterol (Ch).

Bile Acids play an important role in solubilization of cholesterol which itself is virtually insoluble in water $[97,288)$. BA are amphiphilic, possessing both hydrophilic and hydrophobic portions, and form simple micelles in aqeous solutions. A simple micelle is an aggregation of an amphiphilic compound in which the hydrophobic portion faces inward and the hydrophilic group outward, rendering it watersoluble.

BA micelles form spontaneously when BA concentration in bile reaches a certain level, referred to as the critical micelle concentration (CMC) (249).

Other factors influencing the formation of micelles in bile are the concentration of biliary solids, molecular structure of Bile Acids, temperature, $\mathrm{pH}$ and counter ion concentration. Since Cholesterol is insoluble in water its biliary solubility appears to depend solely upon its concentration relative to the molar concentration of $\mathrm{BA}$ and $P P L$, forming mixed micelles. The relative proportions of these 3 lipids, as well as the total lipid concentration, are the main determinants of the degree of biliary cholesterol saturation. The particular species of BA in a mixed micelle probably has only a minor influence on its capacity to solubilize cholesterol.

The ability to dissolve cholesterol increases from glycine-DCA to taurine-cholic acid (164). The greatest differences between the individual $B A$ with respect to cholesterol dissolving capacity occur when no PPL are present. Individual differences decrease as the concentration of lecithin (the main PPL compound) increases, which eventually becomes the dominant factor in cholesterol solubility.

A graphic presentation of cholesteral solubility was first proposed by Small and Admirand in 1968 (5). The relative mols.percentage of the three biliary lipids was graphically presented by a triangle. If the relative concentration of individual bile lipids was estimated the solution could be determined saturated or supersaturated with Ch. A supersaturated solution is known to contain free cholesterol in crystal forms initiating stone formation (321).

At present different numerical expressions are in use to quantitate cholesterol solubility (178) like Lithogenic Index (LI) and Cholesterol Saturation Index (CSI). However, the methods presently available for ascertaining the Ch holding capacity of bile are based upon the analysis of solutions in vitro systems in which the ratio's between the 3 lipids can be varied at will while in vivo only limited ratio's are encountered especially between BA and PPL. These methods represent semiquantitative calculations using diagrams measuring cholesterol solubility. Unfortunately, the original determinations of the individual components of the lithogenecity triangle probably do not correspond with laboratory methods used in different clinical studies. Nonetheless, the original saturation indices are used as a 
reference in calculating saturation index, which will enhance inaccurate results leading to erroneous conclusions. An accurate quantitative index expressing the exact degree of cholesterol saturation seems preferable rather than resorting on a diagram reference established in other laboratories. At present, however, only a few laboratories are engaged in the empirical determination of biliary cholesterol solubility. Although the solubilisation of cholesterol is mainly dependant on the proportion of BA and PPL it is quite clear that bile need not be considered as a simple micellar system with three independant interacting compounds as represented by the triangular phase diagram. BA secretion is known to correlate with PPL secretion: when BA secretion changes, the PPL/Ch ratio also change (291). In conclusion, the difference in the individual components of bile lipids seems more important to look at than to rely solely on one of the numerical and graphic representations concerning cholesterol solubility. This strategy is followed in the clinical study on hepatic bile of gallstone patients (Chapter 14)

\subsection{Cholesterol metabolism}

Cholesterol was discovered in the 18th century from extracts of human gallstones. Later this compound was identified in human and animal tissues of different origin and was also discovered in atherosclerotic lesions.

Comparable steroidal compounds present in plants are generically known as phytosterols, of which $\beta$-sitosteral is the best known. Phytosterols are poorly absorbed. $\beta$-sitosterol competitively inhibits cholesterol absorption and is used clinically to treat hypercholesterolemia as well as experimentally as a dilution marker in cholesterol balance studies (29). Cholesterol is relatively insoluble in water and is perhaps one of the largest molecules to be absorbed readily from the intestinal tract. Exogenous human cholesterol intake amounts to a total of $0.3-1.0 \mathrm{~g}$ per day. It is assumed exogenous and endogenous cholesterol mix indistinguisably in the intestinal lumen and their absorption is believed to be identical.

Absorption of dietary cholesterol occurs mainly in the proximal small intestines after mixing with bile. Mucosa cells most active in cholesterol absorption are those lining the tip and upper halves of the villi. Because of a very high turnover rate of these cells cholesterol absorption is also dependent on their lifespan. This dependence is strengthened because the rate of transport of cholesterol from the intestinal wall to the lymph relative to the turnover rate of mucosa cells is low enough that a significant proportion of cholesterol taken up by the cells may still be contained in these mucosa cells when they are exfoliated in the intestinal lumen. Cholesterol present in these cells is not readily available for reabsorption because cell desintegration takes too long before freed cholesterol can be incorporated in micelles for absorption. This is why only approximately $40 \%$ of dietary, exogenous cholesterol is absorpted. The upper limit of daily cholesterol absorption is estimated to be between $600-1000 \mathrm{mg}$ per day. The unabsorbed cholesterol is acted upon by micro-organisms in the large bowel and converted to a large number of other nonabsorbable steroids before faecal excretion. The predominant faecal metabolite in 
the colon is coprostanol. Biliary secretion of cholesterol is approximately $1500 \mathrm{mg} /$ day. A maximum of $40 \%$ is reabsorbed, the percentage being inversely proportional to dietary intake of cholesterol. Other organs involved in cholesterol catabolism are the skin by cell-loss and endocrine glands which take up plasma cholesterol as a precursor of steroid hormone production, but the bulk of loss is faecal.

\subsection{Cholesterol synthesis}

Cholesterol has a perhydro-cyclo-penta-phenanthrene skeleton (fig. 2.1).<smiles>c1ccc2c(c1)ccc1ccccc12</smiles>

fig. 2.1: Phenanthrene skeleton

Cholesterol has a hydroxylgroup $(\mathrm{OH})$ at carbon position 3 and a double bond between carbons 5-6 (fig. 2.2).

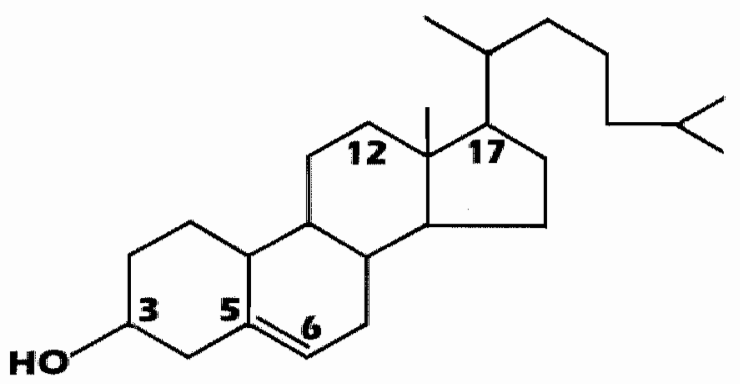

fig. 2.2: Chemical structure of Cholesterol

The first step in cholesterol synthesis constitutes activation of acetate by coenzyme A. Two acetate Co-A molecules combine to form aceto-acetyl Co-A, which molecule serves an intermediate for different metabolites as well as cholesterol. Addition of another acetyl Co-A molecule makes P-hydroxy-P methyl-glutamyl-COA IHMGCOAl. Conversion of HMG-COA to mevalonic acid by HMG-CoA-reductase is an irreversible step of cholesterol biotransformation. The HMG-CoA-reductase catalyzes the rate limiting step of cholesterol synthesis in the liver. Compounds like CDCA and phenobarbital may also influence HMG-CoA-reductase activity, but the mechanism of this effect is not known.

Intracellular free cholesterol exerts a negative feed-back inhibition of HMG-COAreductase. The activity of this enzyme accurately reflects the state of cholesterol synthesis. The same sequence of enzymatic transformation in cholesterol synthesis is 
also found in Gl mucosa as well as the skin.

However, the highest rate of cholesterol synthesis per unit weight of tissue occurs in the liver and intestines. Relative distribution of total body cholesterol synthesis in man is $80 \%$ in the liver, approximately $10 \%$ in Gl tract, mainly ileum, and about $5 \%$ by the skin. The remaining $5 \%$ is synthesized in other tissues. Intestinal uptake of cholesterol is increasingly facilitated by bile acids. Any experimental manipulation of EHC of BA's changes secondarily the enterolymphatic absorption of cholesterol (114). This is confirmed by a marked reduction of cholesterol absorption in the absence of $B A$.

\subsection{Bile Acid synthesis}

Bile acids are steroid molecules derived from cholesterol. They are therefore closely related to the following important and widely distributed substances:

cholesterol, adrenal cortex hormones, sex hormones, vitamin $\mathrm{D}$, digitalis glycosides, ergotamines, sitosterols.

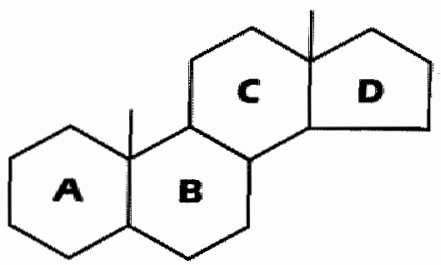

fig. 2.3: Perhydro-cyclo-penta-phenanthrene nucleus

All steroids have a similar cyclic nucleus resembling phenanthrene (A, B, C ringl to which a cyclopentane ring (D) is attached (see fig. 2.3). However, the rings are not uniformly unsaturated, so that the parent substance (completely) is better designed as perhydrocyclopentanophenanthrene nucleus (fig. 2.1). Methylgroups are frequently attached to position 10 and 13 , creating $C$ atoms respectively enumerated 19 and 18. Position 17 is linked to the side chain (see fig. 2.3). Bile Acids exclusively formed in the liver (hepatocytes) from cholesterol are called primary Bile Acids. These are cholic acid and chenodeoxycholic acid (see fig. 2.4). The pattern of 

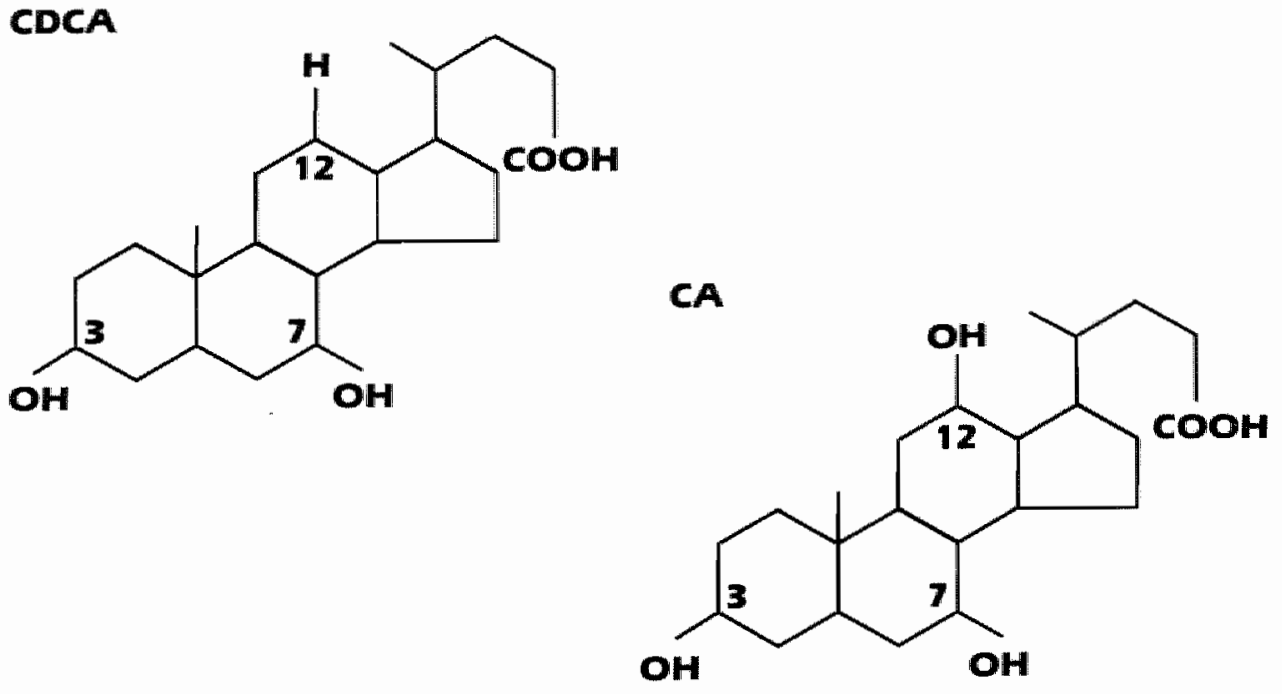

fig. 2.4: Cholic Acid and Chenodeoxycholic Acid

hydroxylation of the steroid nucleus at positions 3,7 and 12 distinguishes the four major bile acids in human physiology. All three positions are hydroxylated in the trihydroxy BA: cholic acid. Chenodeoxycholic acid is a dihydroxy BA with the 3 and 7 positions hydroxylated.

Ursodeoxycholic acid is the $7 \beta$-isomer of CDCA, normally present in small amounts in human bile.

Secondary BA are formed from primary BA by bacterial enzymatic degradation with $7 \alpha$-dehydroxylase in the intestines.
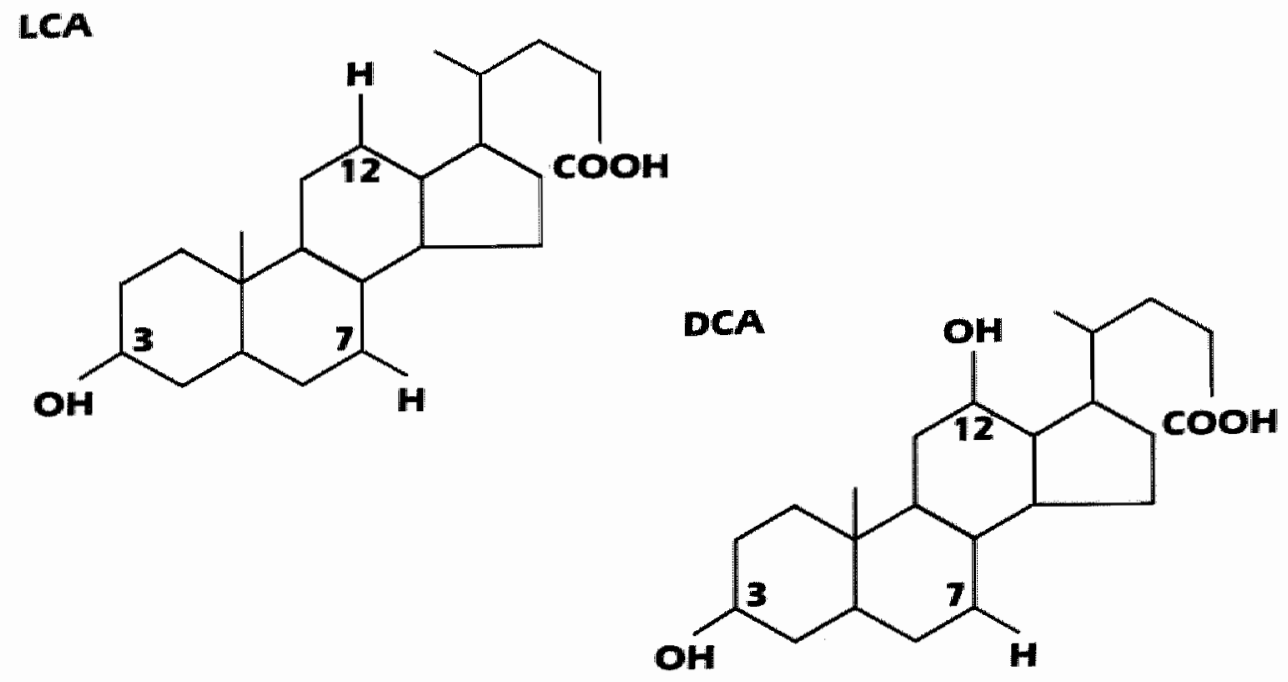

fig. 2.5: Lithochalic Acid and Deoxycholic Acid 
Lithocholic acid is derived fram CDCA and Deoxycholic acid from CA (fig. 2.5).

Tabel 2.1

$\begin{array}{lll}\text { No. OH Group } & \text { (Trivial) name } & \text { Position OH } \\ \text { Trihydroxy } & \text { Cholic Acid } & 3-7-12 \\ \text { Dihydroxy } & \text { Chenodeoxycholic acid } & 3-7 \\ & \text { Deoxycholic acid } & 3-12 \\ \text { Monohydroxy } & \text { Lithocholic acid } & 3\end{array}$

table 2.1: Major BA in human physiology

The four major BA in human physiology are summarized in table 2.1 .

The investigations culminating in the present detailed knowledge of BA biosynthesis have been adequately reviewed previously (30) and will be omitted in this thesis. Only the broad outlines will be summarized here.

Primary BA are predominantly formed from newly synthesized cholesterol in the microsomal structure of the hepatocytes. Cholestero $7 \alpha$-hydroxylase is the ratelimiting enzyme. This reaction is regulated by negative feedback inhibition by BA's returning to the liver via the portal vein. Another important enzyme activity is $12 \alpha-$ hydroxylase, by which cholic acid is synthesized. Both enzyme activities have been located in the hepatocyte microsomes and require co-factors including cytochrome P-450, NADH, ATP.

The average daily production of newly synthesized $B A$ in man is estimated to be 0.6 g (357).

Cholic acid synthesis rate is approximately twice the synthesis rate of chenodeoxycholic acid. The total body pool of CDCA roughlly equals that of $C A$, because the turnover of $C A$ is also greater than that of CDCA.

Lithocholic acid (LCA) is derived from chenodeoxycholic acid by bacterial $7 \alpha$ dehydroxylation in the bowel. The total pool of LCA in man is small, around $100 \mathrm{mg}$. Two factors appear to explain the small size of total LCA pool. First, this BA is poorly absorbed. Second, it is sulfated in the liver, and thereby becomes highly water soluble and susceptible to excretion in the faeces 1941. This BA is hepatotoxic, so sulfatation would appear to be a biotransformation of considerable protective advantage, especially in man since the human is unable to rehydroxylate LCA to CDCA (28). In general, sulfatation is enhanced in patients with cholestasis of different origins, and helps reduce the high concentrations of potentially toxic bile acids.

LCA is a highly cytotoxic substance. This toxic effect results only from free LCA, that is unconjugated and non-sulfated. Slight and reversible liverfunction derangements have been described during Cheno-medication in gallstone patients. This is thought to be due to increased formation of LCA, the secondary BA derived in the gut from CDCA. Sulfatation may protect against LCA-mediated liver damage during Cheno- 
therapy, and may therefore be important in determining which patients can tolerate this form of therapy well.

Ursodeoxycholic acid (UCDA, the $7 \beta$-isomere of CDCA) does not undergo bacterial $7 \alpha$-dehydroxylation and therefore its administration does not result in increased LCA levels. Neither does its administration cause the abnormalities in liver-function tests characteristic of CDCA therapy. This suggests that LCA formation and toxicity are the cause of the mild derangements of liverfunction tests seen with CDCA therapy, and that UCDA, though more expensive, may be a preferable mode of therapy in gallstone patients (Chapter 5).

\subsection{Bile Acid conjugation}

Before secretion, BA are conjugated with glycine or taurine by covalent linkage. Glycine conjugates are normally about 3 times as abundant as taurine conjugates in human bile. In other vertebrates the glycine/taurine ratio is reversed, as compared to humans. In animals resistant to atherosclerosis BA are almost exclusively conjugated with taurine. Moreover, gallstones rarely develop spontaneously in species which conjugate BA primarily with taurine.

Heppner and Hoffman $(170,172)$ reported on physiologic behaviour of dihydroxy$B A$ conjugates with glycine. They found daily fractional turnover glycine moiety of both cheno-and deoxycholic acid to be three times that of steroid moiety. A total pool of $6 \mathrm{mmols} /$ day is used for conjugation in health: 1.8 to conjugate newly synthesized BA and 4.2 for reconjugation. They also measured total BA synthesis which was established to be $2.1 \mathrm{mmol} /$ day. However, with increasing enterohepatic cycles per day $B A$ absorption increased. Because of a distinct difference in glycine and $\mathrm{BA}$ turnover rate, about $15 \%$ of total $\mathrm{BA}$ is reabsorbed unconjugated.

In humans glycine conjugates predominate over taurine conjugates in a ratio of $3: 1$ 4:1. This ratio can be reversed under experimental conditions by prolonged taurine feeding. The known prevalence in conjugation is therefore due to the lesser availabillity of taurine and its precursors.

Gardner (135) gave PABA (Para Amino Benzoic Acid) and TCA (Tauro Cholic Acid) to patients after the seventh postoperative day following cholecystectomy when biliary composition had stabilized. Benzoic acid is converted to hippuric acid, which is efficiently conjugated by glycine thus minimizing total glycine pool availability for $B A$ conjugation. Indeed a reduced but not totally reversed glycine to taurine ratio was established. These results support the thesis that predominant glycine conjugation does not reflect a preferential pathway but rather a deficiency of available taurine in man.

\subsection{Bile Acid pool size}

The sum total of BA in tissues and organs is called the Bile Acid Pool. The BA pool is distributed mainly in the liver, gallibladder, small and large intestines and portal blood. 
BA pool size in health ranges from $2000-6000$ mmol approximately.

Several studies indicate $90-95 \%$ of total BA is located in gallbladder and Gl tract. Abnormal expansion of the BA pool is reported in HLP type N. A decrease in pool size however is seen in cholesterol cholelithiasis and may be of determining importance in cholelithogenesis.

Both gallbladder dynamics and Small Bowel Transit Time (SBTT) independantly affect total BA pool size. A normally functioning gallbladder regulates pool size in that the more vigorously it contracts, the more Bile Acid is put into the EHC where it both feed backs negatively on BA synthesis, and is subject to fecal excretion. The BA pool size therefore tends to vary inversely with gallbladder contractility (115).

A non-functioning gallbladder, as diagnosed by $X$-ray, probably has little if any influence on pool size. Roslyn (301) reported on dynamic changes in gallbladder function and BA pool size during different phases in gallstone formation. Pool size altered progressively during stone development with an index of $40 \%$ decrease as occurs only after pathophysiologic removal of the gallbladder from the EHC, analogous to the choiecystectomy effect. The decrease of BA pool was suspected to be rather an effect than an cause in stone disease.

A decrease in storage capacity of the gallbladder filled with stones may account for the observed reduction in BA pool size. Thurston implanted prosthetic stones in the gallbladder of dogs and could show a significant reduction in BA pool size by bile displacement (380). The hypothesis that a small BA pool precedes and is responsible for the formation of cholesterol gallstones is seriously questioned by these findings. Pool size reduction can thus be considered a secondary phenomenon and is not manifested until late in gallstone disease.

Bile Acid pool size in the GI tract is mainly regulated by SBTT. BA absorption, synthesis and secretion, total ileal absorption surface /decreased either by disease or operationl, total number of EHC cycles a day, all are parameters of SBTT contributing to a dynamic equilibrium of total $B A$ pool size. The rapidity and number of EHC cycles are inversely related to totai $B A$ pool size. The individual $B A$ have differing dynamics within the EHC. Hepatic uptake of BA facilitated by specific BA receptors located on the hepatocyte membrane (1) might be responsible for the individual difference in BA clearance from portal venous blood. Ahiberg (7) estimated individual bile acid concentration in hepatic bile and serum from portal venous and systemic venous blood in patients undergoing elective cholecystectomy for gallstone disease without CDS, to establish differences in hepatic uptake of Bile Acids. Tri-hydroxy bile acid are cleared more rapidly from the portal circulation than are conjugated dihydroxy bile acids as was shown by disappearence of radioactivity after intravenous injection of labelled Bile Acids.

Serum transport of BA is mainly by albumin which binds dihydroxy bile acids more avidly than trihydroxy bile acids: The differences in the availability of free $e_{x}$ or unbound BA, suggest that protein binding is not the sole explanation. Hepatic uptake of the individual conjugated BA is estimated to be about $90 \%$ for CA and $70 \%$ for CDCA.

Also the ileal transport system has known different affinities for the individual BA 
Different enterohepatic circulation dynamics of the primary BA in men seems thus most likely.

Faecal bile acid loss is compensated for by hepatic synthesis in the normal steady state. The liver can increase the rate of BA synthesis approximately sixfold to compensate for these faecal lasses, as in mallabsorption. Loss of feedback inhibition by $B A$ in portal venous blood appears to be responsible for the acceleration of $B A$ synthesis in malabsorption.

\subsection{Intestinal phase of Bile Acids}

Events occur during the intestinal passage of $B A$ which influence the composition, qualitatively as well as quantitatively, of BA pool and the individual BA's.

As described previously secondary BA's are formed by bacterial dehydroxylation. The main enzymatic activity is intestinal $7 \alpha$-dehydroxylase by which DCA is formed from CA and LCA from CDCA. This biotransformation is unrelated to the degree of deconjugation $(171,172)$.

As was measured by breath test (133), glycine turnover rate was established being 5 times that of cholyl moiety of cholylglycin, indicating glycine cycles are 5 times that of cholyl in the EHC. In this study by Hofmann it was also clearly established dehydroxylation was strictly performed by anaerobes and deconjugation by aerobes. Anaerobes are predominantly found in the large bowel. Dehydroxylation is thus strictly performed in the colon and deconjugation in the small bowel.

A change in intestinal microflora can be detected by a change in BA composition as was shown by Low Beer (223), who investigated patients on metronidazol medication during 1 week. He found that the duodenal bile composition was dramatically changed. The secondary bile acid DCA was decreased from 24 to $7 \%$ and reestablished to pre-antibiotic level after discontinuance of metronidazole to $20 \%$. Important observation was that the CDCA increased after 1 week antibiotic medication from 34 to $46 \%$ and again back to $33 \%$ after discontinuance.

If dietary measures can be taken reducing the return of newly formed DCA from the colon of the bile, cholesterol saturation will reduce and with it the risk of gallstones. From other studies bran feeding was established to be one of these measures.

An important factor in grossly maintaining small bowel bacteria counts is intestinal motility. Other factors also involved in a balanced small bowel microbial flora are intraluminal factors like mucus, immunogiobulins, bacterial metabolic products and bile salts. In physiologic concentrations bille salts can inhibit bacterial growth in vitro, particularly certain colonic anaerobes.

However, in an in vivo study Williams could not show significant evidence that bile salts could clear small bowel of colonic anaerobes $|415|$.

The site of bile acid absorption is well established. Two mechanisms are important: passive absorption, mainly in the proximal small bowel and colon (273), and active absorption in the distal small bowel.

Ponz de Leon (1978) measured serum levels of BA post-prandially in patients on BA feeding (279). Peak rise in serum CDCA conjugates was seen within 30 minutes. 
This suggests CDCA may undergo considerable passive absorption in the upper jejunum. Simultaneous injection of CCK promoting more rapid transport of intestinal contents by stimulating small bowel motility, enhanced the early maximum rise in serum CDCA. These findings confirmed proximal small bowel absorption of CDCA. Thus the ratio of intraluminal $C A / C D C A$ increases progressively from proximal to distal small bowel refiecting distribution of passive and active transport mechanisms alongside the small bowel. If the SBTT is increased, the BA composition of bowel contents reaching the large bowel show a high $C A / C D C A$ ratio. Dehydroxylation is thus predominantly performed on CA. An increased proportion of DCA in the BA pool may thus be indicative of an increased synthesis rate resulting from an extended SBTT (298).

Deconjugated BA are passively absorbed more readily than conjugated BA (136). This may be important quantitatively in blind loop syndromes when stagnant bacterial jejunal contamination leads to rapid deconjugation of $B A$.

Free $B A$, especially DCA, is known to cause intestinal mucosal cell damage even in physiologic concentration. CDCA on the other hand has no similar effects on the mucosa at physiological concentrations.

Intractable diarrhea following gastric surgery and more frequently reported when combined with cholecystectomy, is mainlly thought to be choleric in origin. $B A$ are supposed to interfere with $\mathrm{H}_{2} \mathrm{O}$ resorption in the colon. Mehkjan (245) investigated water resorption relating to $\mathrm{BA}$ and could show a relationship between chemical structure and secretory activity: preferably dihydroxy BA whether free or conjugated, inhibit absorption and induce reversible secretion of water and sodium ions. The response was concentration-dependant. The established BA induced colonic secretion of $\mathrm{H}_{2} \mathrm{O}$ and electrolytes is mediated by 3,5 cyclic-AMP, but also effected by different hormones and even enterotoxins, like E.Coli and V.Cholerae $(370)$. Conley (90) found a simultaneous increase in phospho-diesterase activity (PDE] in colon mucosa, the enzyme which degrades $3^{\prime}, 5^{\circ}$ cyclic-AMP. However, the biologic significance of the measured net increase in PDE activity was too low to exert al significant inhibiting effect on cellular cyclic-AMP levels.

It must be clear that intestinal disease with severe mucosal damage will have repercussions on $\mathrm{BA}$ absorption and consequently $\mathrm{BA}$ pool composition.

Marks (234) established lithogenic indices of duodenal bile samples in inflammatory small bowel disease. It is interesting to know that cholesterol saturation did not correllate with ileal resection nor the extent, duration or activity of ileitis itself. However, he could establish significant differences of the lithogenic indices among normals, patients with ulcerative colitis and patients with ileitis.

Results in both ileitis and ulcerative colitis patients suggest the interruption of EHC caused by small resection and/or ileal disease is significant enough to increase biliary cholesterol saturation being definitive a prerequisite for gallstone formation. This is in accordance with the reported high prevalence of gallstones in these
patients. 


\subsection{Postcholecystectomy dynamics in BA metabolism}

To gain insight into the etiology of gallstones many studies have been performed in the direct postoperative period following cholecystectomy. In many gallstone patients the gallbladder is already short circuited in the EHC because of nonfunction, established by oral cholecystography. The postoperative dynamics on BA pool particularly in these patients could thus already have been present preoperatively $[289,290)$.

W/hether or not bile composition improves postoperatively following cholecystectomy has not yet been definitely settled $(242,346)$. Wide variations in patient selection is the major denominator for the conflicting results so far published. Methodologic differences in bile sampling, diurnal variations in bile composition, hormonal variations during menstrual cycles (224), age and the duration and extent of gallstone disease, the influence of drugs and hormones all may contribute to uneven results.

\section{BA dynamics in the post-cholecystectomy state}
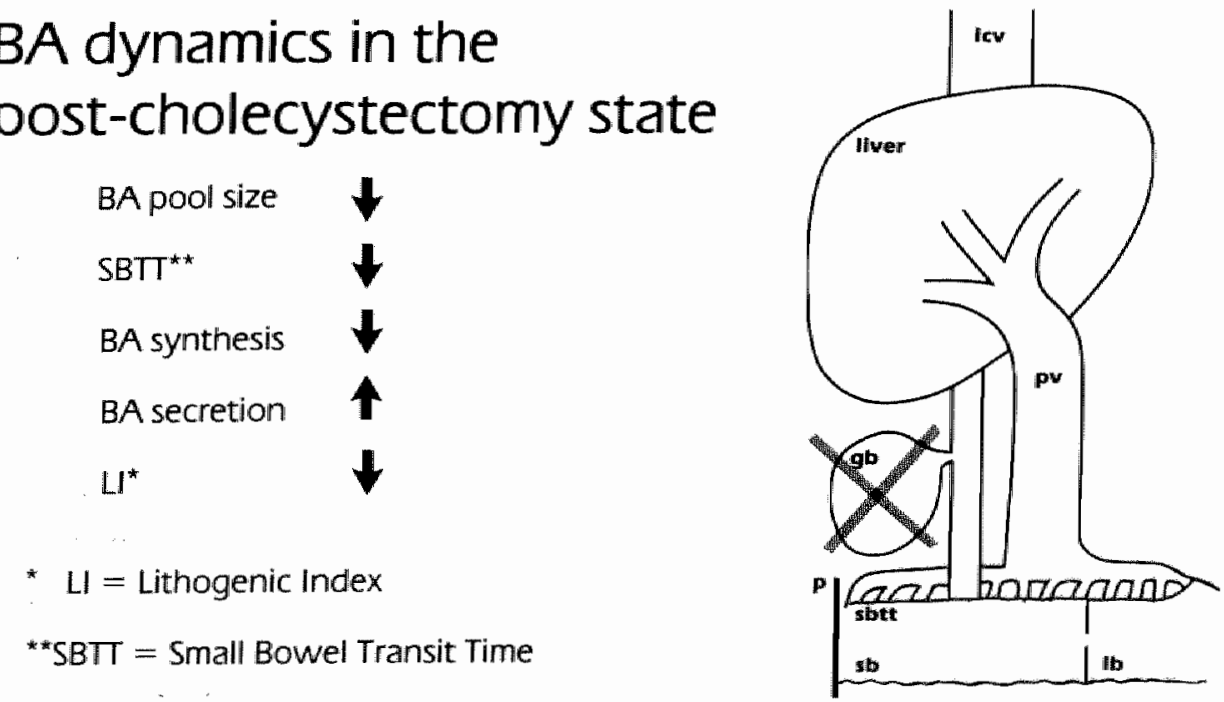

However, distinct differences in pre- and postoperative bile composition are noted. The gallbladder loss in patients who underwent cholecystectomy is compensated for by a redistribution of total bile acids pool being mainly manifested by an extended intestinal BA pool phase (298). This is followed by a change in the relative composition of total BA. The portion of secondary BA increases because of microbiologic and enzymatic activities in the intestines. BA pool size in postcholecystectomy patients correlates highly with the size of secondary BA pool (170). This mostly concerns DCA because LCA is expelled from the EHC by sulfation followed by faecal excretion. DCA concentration, measured by breath test, is increased in the gallstone patient.

After cholecystectomy and re-establishment of EHC, hepatic bile contains less DCA, indicating a more efficient EHC, with less BA spillover in the colon. 
Also Shaffner found improved biliary cholesterol solubility in postcholecystectomy patients (331).

The observed extended enteric $B A$ pool was also confirmed by Duane (114). He found SBTT of BA was accelerated two or three fold by administration of nonabsorbable cholesterol or alcohols, similating the defect in BA metabolism in gallstone disease.

The increased removal rate of $B A$ from the small bowel by increasing frequency of EHC cycles does not reduce total flux of $B A$ through the liver.

The observed defect in BA kinetics in the postcholecystectomy patjents is not accompanied by a rise in synthesis but rather a slight decrease, because venous portal BA concentration is raised (causing a negative feedback inhibition on BA synthesis). With this model a net decrease of $27 \%$ of total BA pool was established by reducing SBTT from 86-44 minutes. This decrease in BA synthesis does not necessarily lead to a lower BA output because synthesis contribute only about $5 \%$ of total BA output. Total BA output is slightly raised in the postcholecystectomy patients because of increased cycling rate due to loss of the sequestering action of the gallbladder.

in conclusion a new steady state is created in BA metabolism in the postcholecystectomy patients (see Table 2.2):

The loss of the gallbladder contributes to a decrease in BA pool. This is efficiently dealt with by an increased frequency of EHC cycling, and a shorter SBTT.

Total BA flux per day through the liver is increased per volume unit of bile.

$B A$ synthesis is decreased by negative feedback mechanism mediated by a raised $B A$ concentration in portal blood.

So in spite of a decrease of total BA pool size a rise in biliary BA concentration improves cholesterol solubility in the postoperative period.

\subsection{BA synthesis during biliary obstruction}

In obstruction no $B A$ returns to the liver. $B A$ synthesis becomes maximum by negative feedback mechanism. The retained $B A$ pool in the liver suppresses synthesis by blocking cholesterol $7 \alpha$-hydroxylase but equilibrium is reached with high $B A$ pool levels in the liver.

Only $10 \%$ of total BA pool is lost in urine after sulfatation. Redinger $(292)$ found $B A$ synthesis was suppressed during obstruction and remained inhibited up to 10 hours following reinstitution of EHC in the postobstruction period. It is curious to note that PPL excretion was also increased in the immediate postobstruction period because PPL and BA are supposed to be closely related in secretion and probably in synthesis as well. Serum BA and urine levels became neglectable after 24 hours following relief of obstruction, suggesting tissue binding of BA is not a major factor. $\mathrm{CA}$ is the predominant serum $\mathrm{BA}$ in acute biliary obstruction. The decrease in cholesterol dissolving capacity of bile found in these experiments $(292)$ indicate that intermittent obstruction like in CDS aggravate and perpetuate gallstone formation and/or growth by favouring cholesterol synthesis in the face of decreased bile acid 
synthesis! Important facts for a thorough discussion on the surgical dilemma of recurrent or reformed stones will be dealt with later on (Chapter 14 ).

A typicall change in bile lipid pattern during and immediately following obstruction was established by Ekdahl (120). A considerable decrease in DCA was noted because intestinal dehydroxylation was blocked. Established hypersecretion of $B A$ lasted for 4 days after relief of obstruction. Following operative relief of obstruction, bile volume as well as bile acid secretion increased with a slight elevation in biliary CA during some days of rehabilitation. In biliary obstruction in rats, alternate pathways of BA metabolism could be established by Greim (153) protecting intracellular synthesis structures from detergent BA action. This reduces hepatotoxic effect in $B A$ retention during biliary obstruction.

Histologic correlation between dehydroxylated BA levels and cholestatic liver cell injury in humans has been established (152). Consequently, high levels of trihydroxylated BA, that is Cholic Acid, are established in human livers in case of obstruction. The mechanism whereby CA becomes a greatly predominant endproduct of BA synthesis during extrahepatic biliary obstruction is not known. Postobstructive bile composition will thus show a high CA concentration as long as hepatocellular storage is not depleted and synthesis not totally reinstituted. In obstructive cholangiopathies plasma CA will thus predominate. Under normal physiologic conditions serum CDCA concentration is higher while in chronic parenchynal liver damage CDCA becomes the major serum BA (20).

Serum BA levels reflect BA synthesis which can thus be reliably tested in differentiating obstructive jaundice.

\section{$\therefore$ Bile formation}

Bile formation is a complex process involving secretory processes taking place at the canalicular membrane. Cytoskeleton of the bile canalliculus is closely linked to the intracellular architecture of the hepatocyte. Microfilaments containing actin and other contractile proteins are forming a peri-canalicular ectoplasm inserting into the core of microvilli in the canaliculus as well as into the cytoplasmic surfaces of plasma membrane. The bile canaliculus constitutes only 10-15\% of total hepatocyte membrane where the tight junction between adjacent cells are delineated by the zona occludens (241).

One canalicular process is the active transport of organic ions from hepatocyte to canalicular lumen. The osmotic gradient resulting from organic ions transport causes the movement of $\mathrm{H}_{2} \mathrm{O}$ and inorganic electrolytes to the canalicular lumen. The most important substances involved in this process are the bile salts. Hence this process is referred to as Bile Salt Dependent Canalicular Bile Flow (BDCF).

There is in addition another canalicular secretory mechanism thought to be related to canalicular $\mathrm{Na}^{+}, \mathrm{K}^{+*}$ ATPase and unrelated to Bile Salt Secretion.

This portion of canalicular secretion is called: Bile Salt Independent Canalicular Bile Flow [BICF].

A third contributing mechanism in Bile Flow is Ductular secretion stimulated by 
gastrointestinal hormones, like: gastrin, secretin, cholecystokinin (332). None of these hormones has been demonstrated to stimulate the bile salt independant canalicular bile secretion (18).

Relative total amounts of the different fractions in bile formation are:

BDCF: $225 \mathrm{ml} / 24$ hours

BICF: $225 \mathrm{ml} / 24$ hours

DBF : $250 \mathrm{ml} / 24$ hours

Total: $600 \mathrm{ml} / 24$ hours

\subsection{Bile Salt Dependant Canalicular Bile Flow}

Bile saits are actively secreted against a high concentration ratio. The physiologic basis for the lineal relationship between canalicular bile flow and BA secretion is assumed to be related to the osmotic properties of the $\mathrm{BA}(190)$.

Whether BA are secreted as mixed micelles or as individual molecules is still a controversial issue. BA which form poor micelles, such as DCA, are more active in canalicular bille production suggesting micelles are only formed in the canaliculae before the secretion of the individual components of mixed micelles.

BDCF can be influenced by the vagus nerve. MacDonald did indeed found an increase in bile salt output and concomitant bile flow in dogs by insulin hypoglycaemia induced by vagus stimulation (241). The observed differences of one hour between peak choleresis and maximum insulin effect raise a possible indirect effect by vagal stimulation rather than a direct effect on the liver and warrants cautious interpretation of this study on vagal factors in bile flow. The generation of canalicular bile flow is reduced by estrogen. The mechanism appears to be a change in cell permeability for $\mathrm{BA}$ resulting in a decrease in BDCF leading to estrogen induced physiologic cholestasis.

Because BA"s also play a major role in regulating biliary secretion of both cholesterol and PPL, factors influencing BDCF seem more important considering lithogenic bile production, because total water content plus the relative lipid composition are changed simultaneously.

\subsection{Bile Salt Independant Canalicular Bile Flow}

The sodium pump is considered the leading physiological mechanism for the generation of BICF. All mechanisms postulated for water movement across membranes in biologic systems are involved in bile flow as well. These include: pinocystosis, active water transport, passive water flow following osmotic or hydrostatic gradients. As far as passive water flow is concerned, osmotic pressure seems more important than hydrostatic pressure perfusing the liver: sinusoidal pressure was less than intracanalicular pressure produced by bile duct ligation but nevertheless bile continued to flow (190). The activity of $\mathrm{Na}^{+}, \mathrm{K}^{+}$- ATPase, the enzyme responsible for active sodium transport across cell membranes, is most likely
to play a central role in bile flow.

Several substances which stimulate cyclic-AMP formation in the hepatocyte 
including glucagon, phenobarbital and theophylline, increase canalicular bile flow. Ouabain however may inhibit the intracellular sodium pump resulting in a reduction of canalicular bile flow.

Prostagladins are known to increase renal sodium and water excretion, and may operate in the bile canaliculus as well, although the exact place of prostagladins in billiary dynamics (and chemistry of bile lipids) is still unknown (392).

In general, changes in BICF have little or no effect on bile composition regarding cholesterol solubility: the relative concentration of the main bile components are equally effected by a change in BICF, which thus seems of mimor importance in cholelithogenesis.

\subsection{Vagus influence in bile salt secretion}

Extensive literature is available on the importance of the vagus nerve in gallstone formation $(23,64)$, but mostly concerns the role of the gallbladder rather than bile lipid composition. Although contrasting data are available on bile lipid composition, extensive clinical studies have not yet been performed on the effect of the vagus activity and BA composition.

Jones (328) could not establish a change in ductal secretion after vagotomy in animals, although previous findings show a marked increase in bile flow mediated by insulin hypoglycemia on ductal secretion.

However, the only well established effect on biliary dynamics of vagus activity is its effect on gallbladder tonus which changes bile lipid composition, because of an increased volume of sequestered bile in the distended gallbladder.

Atropin has been established to produce a significant fall in bile volume and secretion of all three major bile lipids, so the vagus indeed has a stimulatory effect on bile salt secretion and flow (196). This may result in a change in cholesterol dissolving capacity.

Carey and Small (77) have shown that over the physiological range of total lipids per volume of bile $\uplus 0.2-25.0 \mathrm{~g} /$ deciliter) the capacity of bile to solubilize cholesterol is increased. Dilute bile is thus less capable of cholesterol solubilization than concentrated bile of the same relative lipid composition (37). Although hepatic bile may become progessively more saturated during an overnight fast because of low secretion rates of BA (49), gallbladder bile simultaneously may become more capable of cholesterol solubilization because of the concentrating action of the gallbladder mucosa.

Sier (341) could not establish a change in biliary pressure in dogs after vagotomy. His results contrast with those of Schein (Chapter 8: CBD), who found an obligatory fall in pressure in the biliary system across both pars spiralis and Oddi's sphincter in dogs and humans.

The negative findings by Sier are of paramount importance in considering his concomitant results on bile acid composition after vagotomy. He claimed the 
extrahepatic compartment of EHC to be responsible for the change in BA composition after vagotomy.

However, the reported experimental results suggest a longer SBTT or/and extended colonic phase, because secondary BA were raised, possibly induced by a lessened intestinal motility with altered microflora.

Equivalent clinical investigations have not yet been performed. in fact, so far there has been no convincing vagal factor discovered in man relating to a change in bile lipid composition responsible for cholesterol stone formation, other than altered gallbladder motor function. 


\section{CHAPTER 3}

\section{Associated metabolic and endrocrinologic disorders}

\subsection{Diet and gallstones}

Geographic distribution of gallstone disease gives substantial evidence that diet plays a dominant role in pathogenesis. The composition of western diet shows an increase in relative concentrations of carbohydrates and fat, mainly of animal origin and a decrease of bran. BA metabolism has been subject of extensive sududies to show the different infiluences of variations in diet compositions. The increase of fat intake is dealt with by an increased secretion of biliary lipids, mainly $\mathrm{BA}$ for fat breakdown and absorption. It is well established that an increase in faecal BA loss is initiated by a high fat intake, while excessive long chain fatty acids may inhibit BA reabsorption. Consequently, the total amount of $B A$ in the EHC is increased, to compensate for the intestinal loss and extended small bowel transit time (SBTT). The rise in $B A$ in the EHC is mainly accomplished by raised colonic intraluminal levels of $B A$ as can be measured by the breath test (133).

A high unsaturated fat diet has been extensively promoted recently, for prevention of complications of generallized atherosclerosis, mainly in coronary heart clisease. By autopsy studies, exclusively on male patients, Sturdevant (364) established an increase in gallstones in patients particularly using this diet.

The relative biliary lipid composition changed during the unsaturated fat diet. The estimated decrease in biliary CDCA might favor precipitation of cholesterol, possibly explaining the increased prevalence of gallstones by this particular cholesterol lowering diet.

In the just mentioned autopsy study, a significant increase in gallstones was noted in obese male patients, both with and without the polyunsaturated diet. 50 the reported high gallstone risk in morbid obesity does not only apply to female adults. An additional effect of the polyunsaturated fat diet apart from its serum cholesterol lowering effect, is an increase in faecal BA output. Considering the hypothetical relation between coloncancer and high faecal $B A$ output, it might be worth reconsidering the widely offered advice to eat polyunsaturates maintaining a low plasma cholesteroll level.

Madura (231) has investigated selected patients with morbid obesity undergoing small bowel bypass surgery, pre-and peroperatively. The high incidence of cholelithiasis lends credibility to obesity being one of the most important determinants in cholelithogenesis, which can only be brought about by exaggerated food intake, high in cholesterol.

Although precise mechanisms have still to be settled. BA metabolism is supposed to play a central role in coloncarcinogenesis becaldse in low cancer risk areas total faecal $B A$ and its metabolites output are reduced.

Reddy (293) found a significant increase in faecal neutral sterols of animal origin in both coloncancer and adenomatous polyps, a precancerous condition. Litho- and deoxycholic acid were significantly raised compared with matched control patients. The changes in biochemical constituents of faeces analysed were secondary and do 
not reflect a causative factor in stool composition prior to the colonpathology, but are at least in some way related to coloncarcinogenesis.

If a rise in associated faecel enzymes, namely 7-a-dehydroxylase and cholesterolhydrogenase, provide causative explanation for the high cancer risik, has still to be confirmed.

However, no major biologic difference in the composition of faecal microflora was noted in patients with low and high fat diets.

Goldberg reported on biologic bacterial analysis of intestinal microflora in Seventh Days Adventists (1 46). He could not show a significant change in faecal microflora. This might explain differences in colonic micro-organisms do not have any influence in coloncarcinogenesis.

However, this does not invalidate the concept that dietary animal fat distinctly increases $B A$ degradation within the Gl tract, a factor which has been related to coloncarcinogenesis.

Cholelitholysis treatment by oral BA medication undoubtedly will increase faecal BA output. So far this therapy seems successful in a minority of young low risk patients. with high recurrence rates (Chapter 5).

However, a more comprehensive randomised prospective trial should be carried out to establish any increased risk of large bowel cancer on long term cheno medication before this widespread accepted drug therapy can be justified.

The high feacal BA output and decreased pool size of BA are defenitely gallstone disease characteristics, although the reported incidence of gallstones is not significantly raised in colon carcinoma patients, contrasting to other pathology of the colon, for instance diverticular disease.

Fiber deficiency in the composition of Western diet should also be considered of etiologic importance in both cholelithiasis and coloncancer.

Watts (403) found indeed a lower lithogenic index (LI) of gallbladder bile, by adding bran to a standard diet. Dietary bran increased the relative concentration of chenodeoxycholic acid in bile, which means biliary cholesterol remains unsaturated with no free cholesterol crystals.

In contrast to dietary fat, cholesterol is not a major determinant of faecal BA excretion, because an increase in cholesterol intake has no effect on faecal BA output.

Maudgal (238) reported a low cholesterol diet was supposed to have a decrease in Saturation Index (SI) of gallbladder bile only during cheno medication. However, a thorough analysis of the diet composition in his study concerning total fat content the reported results are more likely to be caused by a concomitant decrease of diet fat percentage, which could have been shown if faecal $B A$ were estimated simultaneously.

\subsection{Hyperlipoproteinemia}

Hyperlipoproteinemia (HPL) has been extensively studied recently, in view of its high correlation with generalized atherosclerosis especially in coronary heart disease 
(CHD). Only in type IV HPL (classification of Friedrickson), occlusive CHD can be prevented by lowering plasma cholesterol and triglycerides by clofibrate therapy 127!

Clofibrate treatment is known to influence serum lipids mainly by increasing cholesterol biliary output with a concomitant fall in BA output (134). Furthermore in primary HPL, BA and cholesterol metabolism was noted to be abnormal: the ratio cholic acid/chenodeoxycholic acid is usually reduced. The clinical-epidemiologic report (Boston Drug Survey Study) strengthens the thesis the increased cholesterol saturation of bile during administration of clofibrate reflects an increased propensity for cholesterol gallstone formation (92). However, thus far, disappointing results in established heart disease have created scepticism about the benefit of drug therapy in lipid disease. So both beneficial and adverse effects of this agent should be kept in mind before starting drug therapy.

Pertsemlidis (276) investigated 10 postcholecystectomy patients to establish the influence of clofibrate treatment. A transcystic catheter was left behind in the biliary duct to collect the samples of hepatic bile at different times during the day.

Consistent changes in the main lithogenic constituents were found: cholesterol was raised and $B A$ concentration was decreased. Changes in the concentration of biliary phospholipids were small and variable, no significant effect was found. A similar but smaller effect on the hepatic bile composition was found during the administration of sex hormones. The alterations in biliary lipids induced by these drugs were independant of pre-existing levels of serum cholesterol. CA kinetics were also investigated: a diminution of both daily synthesis rate and CA pool size was found. A concomitant decrease in faecal BA output was established.

These changes definitely favor the formation of cholesterol gallstones in users of clofibrate and female sex hormones.

Liver biopsy studies, were also performed by Ahlberg (8). The elevated HMG-COA. reductase activity in the liver in type $\mathrm{N} \mathrm{HPL}$, may partially explain the elevated synthesis of cholesterol often seen in these patients.

So it is concluded that clofibrate therapy markedly increase biliary cholesterol content and thereby becomes a potent lithogenic agent in patients not otherwise especially prone to gallstones. Since its role in prevention of ischaemic heart disease is now uncertain, its continued wide use warrants close scrutinity in regard to a high gallstone risk.

\subsection{Diabetes Mellitus}

The mechanism underlying secretion of saturated bile is the only aspect of pathogenesis of gallstones about which there is any understanding. Nonetheless, the Lithogenic Index (LI) is widely accepted being an obligatory parameter for gallstone disease. If a predictable value for stone development can be attributed to LI, DM is very unlikely to constitute a high risk group for stone disease (231), which is in accordance with our clinical results [Chapter 13]. Insulin treatment may also induce the formation of lithogenic bile. 
The only effect of insulin in bile production so far is its strong choleric action. This was thought to be vagally induced, because anticholinergic medication could prevent this response. Additional evidence has demonstrated that insulin has a direct effect on BICF (353). The concentration of cholesterol and BA are supposed to be proportionally decreased in BICF with no effect on cholesterol/BA ratio. Insulin treatment does raise cholesterol concentration in the hepatic bile by decreasing BA synthesis (36).

Bennion (36) made an extensive metabolic balance study to establish a relation between DM and gallstone disease. He studied Pima Indians, a nonrepresentative patient group because of the high predominance of DM (34). His patient group shows racial as well as diet differences with the average western population suffering from gallstones. Different Indian groups also show a high predominance of gallstone disease (157). However. Bennion found no lithogenic gallbladder bile in the untreated, that is hyperglycaemic diabetics. During insulin treatment, $B A$ synthesis was decreased, resulting in a small BA pool. Relative proportions of the four major species of BA however, did not show a significant change in treated and untreated patients. Cholesterol synthesis was high but balanced by an also increased BA synthesis. So if DM is indeed related to a high predominance of cholelithiasis, these results do not show any evidence for support.

Insulin dependent diabetics show a reduction in total BA, but a causative role for insulin remains a matter of continued investigation. Moreover, a contracted BA pool is supposed to be only a secondary phenomenon in stone disease.

The only frequent reported common denominator in DM and cholelithiasis is obesity, which is associated with a high lithogenic bile production by an enhanced cholesterol synthesis. Besides, maturity onset DM is promoted by obesity. which strongly suggests a direct relationship with cholesterol metabolism.

Also other hypoglycaemic medications have been investigated for a possible influence on bile lipid production. Caspary (80) has shown treatment by biguanides only increases BA deconjugation from bacterial overgrowth, with no effect on cholesterol metabolism.

Also predominance of gallstone disease related to age is not different in patients with and without coexistent D.M.. This strongly suggests pathogenesis of DM and cholelithiasis have no common etiologic denomination at all. On the contrary, there is good reason to consider obesity a common etiologic denominator for both DM
and gallstones.

\subsection{Parathyroid hormones}

Although thyroid hormone has a certain influence on steroid metabolism located in whe hepatocyte, hyper- nor hypofunction of the thyroid gland is associated in any gallstones in hyperparatic stone disease. This is in contrast to an increased incidence of widely accepted being yroidism, although these two clinical entities are not as 
of the parathyroid gland. Stone-incidence in hyperparathyroidism increases. significantly to $25 \%$, compared to a $10 \%$ stone-incidence in the general population (330). Age distribution and female predominance are different too from those seen in cholelithiasis: the patients tend to be younger and sex ratio is just about equal. However, stone analysis show predominantly calciumbilirubinate, being more radiopaque than are mixed or pure cholesterol stones. So, symptomatic stone disease, albeit non-cholesterol in origin is a relatively important complication in hyperparathyroidism and ranks third in sequence following the two most frequently and well known clinical manifestations being urinary stones and peptic ulcer.

Pancreatitis constitutes a much less frequently reported manifestation in hyperparathyroidism being reported 4 to $7 \%$, which could also be biliary in origin because CDS are more readily seen, once gallstones are present in hyperparathyroidism (71).

In conclusion, once stones are located on a plain abdominal X-ray, hyperparathyroidism should be suspected and excluded preceding cholecystectomy.

\subsection{Sex hormones}

The female predominance of galistone disease is well known and described as: fat, fair, fertile, female of forty.

The average sex ratio shows a 4: I female preponderance especially in women of childbearing age. The postmenopausal ratio declines to an almost equal distribution with men.

As mentioned before also racial and diet related factors are of importance in the geographic distribution of the disease. That is why different American clinical studies have been performed in South-Western female Indians with their high predominance for gallstone disease in the third and fourth decade of life $(368)$. So it is quite clear that femalle sex hormones are of major importance in gallstone pathogenesis. Principally, two groups of steroid hormones are involved: oestrogen and progesterone. The overall hormonal effect in cholecystolithogenesis resides largely, if not entirely with the oestrogen component of sex hormones. The frequency of stone development in pregnancy is very high, although in some clinical studies only 3 out of every 4 patients operated upon had been pregnant previously (143).

Different authors claim the very beginning of gallstone disease to start in the second trimester of the first pregnancy. This is not surprising because in pregnancy progesterone reaches 20-40 times the normal hormone level and oestrogen even a 1000 times the normal level compared with the nonpregnant state. Plasma cholesterol and triglycerides levels are also raised during pregnancy, which could be of additional importance to an already increased risk of gallstone disease in pregnancy. Data on bile lipid composition in hepatic and gallbladder bile in pregnancy are not widely available. Bennion $(35,39)$ could recently establish that several of the effects of oral contraceptive medication on BA pool size, cholesterol secretion, relative $\mathrm{BA}$ composition and serum triglycerides appear to be totally 
opposite those in patients on chenodeoxycholic acid therapy. Primary BA feeding in patients on oral contraceptives warrants clinical trial to correct the increased lithogenecity.

Acute cholecystitis is often the first clinical manifestation of galistone disease in pregnancy (312). Glenn found that in 1 out of every 5 pregnant women with symptomatic stone disease, acute cholecystitis was clinically diagnosed and confirmed by pathologic examination. CDS were only recovered in $7.8 \%$ of the patients operated on electively or in the acute phase, which was only necessary in a minority of the patients.

Only in 3 out of 29 patients CDS was associated with jaundice which is of importance in differentiating cholestasis of pregnancy from biliary stone disease. A causal relationship between the use of oral contraceptives and gallstone disease has also been proposed.

Leissner (215) has performed a statistical study in women 4 years preceding and 6 years after the introduction of the contraceptive pill in Scandinavia in search for a correlation with gallistone disease. Although no statistical evidence could be established, he found an increased relationship between the use of the contraceptive pill and gallstone incidence in the population investigated. The incidence of cholecystectomy was raised in the age group using the contraceptive pill. This is in accordance with the results of the Boston Drug Survey Control Study 161.621 , which found a $20 \%$ prevalence rate of patients treated with oral contraceptives, most pronounced in the younger age groups.

The overall age-standardized relative risk percentage was calculated to be $20 \%$. Even if oral contraceptive medication was taken less than 1 year, there was a pronounced risk for gallbladder disease.

Since the study was performed low dose pills are more common and the total number of women using the pill has diminished because of other serious longterm side effects. To compare different studies concerning contraceptive medication and gallstone disease the relative amount of oestrogen and progesterone should be noted because in the late seventies the low-dose oestrogen pill was more widely distributed. It remains to be determined whether the low-dose oral contraceptives will show as marked an association with gallbladder disease as their higher dose predecessors.

Reduced total BA pool size is supposed to be a characteristic of gallstone disease. Recent studies have found key evidence this concerns only a secondary manifestation and seems well correlated with the total gallbladder volume. Zunkel has established a marked increase in total BA pool especially in the second and third trimester of pregnancy, equivalent to a $2-3$ times increase in both fasting and postfasting gallbladder volume as measured by ultrasound (425). This strongly suggests
a progesterone mediated phenomenon.

Special attention is paid to oestrogens because suggestive evidence exists it has a strong influence on bile lipid composition. In animal studies, but also confirmed in human physiology, oestrogens interfere with canalicular bile flow, especiaily inhibiting BICF (157). This correlates with cholestatic jaundice in pregnancy, which is 
manifested mainly in the third trimester of pregnancy, when oestrogen levels are high.

Biochemical estimation of the hepatic enzymes and the serum BA gives substantial evidence, the primary disorder in this disease being oestrogen mediated: plasma enzymes are relatively normal especially gamma-GT and also SGOT and SGPT, but. serum $B A$ is raised.

Biochemically this disorder resembles the pathologic phenomena encountered in oral contraceptive medication. Besides, there is a correlation between liver disease and oral contraceptives and recurrent cholestasis of late pregnancy. Once liver pathology during oral contraceptive medication is established, the patient is prone to develop idiopathic cholestasis in a following pregnancy. This all point to an increased sensitivity to oestrogens as is manifested by reversabie liverdamage. In conclusion, the male-femalle difference in prevalence rate in gallstone disease, especially striking in the female reproductive years, is directly caused by hyperoestrogenemia, interfering in bile lipid secretion in the bile canaliculae.

Volume expansion and deminished contraction of the gallbladder, resulting in bile stasis and increased BA pool size, are patho-physiologic phenomena mediated by progesterone but are probably of additional importance in cholecystolithogenesis in the female adult.

Oral administration of female hormones as postmenopausal substitution therapy, as hormone cancer therapy, or as contraceptive medication potentiates the preexistent high risk for cholesterol stone disease in the female adult. 


\section{CHAPTER 4}

\section{Associated pathology}

\subsection{Acalculous Cholecystitis}

The incidence of this entity is relatively rare but more and more cases are reported. The diagnosis made at operation may sometimes be incorrect because small calculi have escaped detection and might be discovered by the pathologist.

Late diagnosis by lack of clinical symptoms or inconclusive radialogic and biochemical assays contribute to a progress of the disease before proper treatment is initiated while increasing the morbidity and mortality significantly.

Acute acalculous cholecystitis is sometimes seen in the postoperative period following major abdominal surgery in the elderly patient (14). It can also be manifested in trauma patients with multi-organ injuries. In burn patients the disease occurs also with increasing frequency (116). Histologic examination of these gallbladders displays the whole spectrum of inflammatory changes from acute to chronic infections. Four main factors have been blamed for:
a. physiologic inactivity of the gallbladder
b. altered bile concentration and/or viscosity
c. vascular derangement
d. infection

a: In the severly traumatised patients with abdominal trauma total parenteral nutrition is institued (274). The Gl hormonal influence of gallbiadder activity in thus absent.

b. Massive haematoma due to multiple factures and/or large retroperitoneal haemorrhage are followed by a breakdown of haemoglobine which in turn increases bile viscosity.

Postoperative dehydration also can lead to inspissated bile resulting in a functional obstruction by the "plugging effect" of the cystic duct (18).

c. In hypovolemic shock redistribution of the vascular compartment to vital organs, resulting in low perfusion rates in other areas like the gallbladder, is often seen. In the elderly patient with an underlying systemic atherosclerosis, hypotension is contributed to the deterioration of the blood supply of the gallbladder wall. Sympathetic stimulation in shock and in the postoperative period can in case of hemodynamic imbalance result in further vasoconstriction.

Ligation of the hepatic artery can also have an impact on the circulation of the gallbladder 1116 .

An ischaemic gallbladder wall contribute to a rapid detoriating clinical picture with gangrene, necrosis and subsequent perforation. These sequelae call for rapid
surgical intervention.

d. Bacterial invasion is not the initial etiologic factor but rather a complication superimposed on an already injured organ.

Acute vascular allergic tissue reaction, the Schwarzmann phenomenon 110,1411 . 
may be triggered by toxines, enhancing the deteriorating pathologic condition in the wall of the gallbladder.

A simple cholecystectomy in acalculous cholecystitis can be curative. Delay in surgery can contribute to a much higher morbidity and mortality. The most important measure is to be aware of the existence of this entity in trauma or in the postoperative phase.

\subsection{Cholesterosis and gallstones}

Gallbladder abnormalities called cholecystoses were described by Jutras in 1960 (193).

Although cholesteral gallstones and cholecystosis constitute two different pathologic entities, they can be found in the same patient discovered on oral cholecystograms (24).

In literature, hardly any information on either the composition of these stones nor on the biliary lipids are available. Recently some comparative studies were reported (48). It was shown that the composition of gallbladider bile was not different from a control group. The exact mechanism for cholesterosis is still poorly understood. In the clinical presentation of chalesterosis, Salmenkive reported jaundice in $10 \%$ of patients (308), which is rather high, compared to the incidence of jaundice in choledocholithiasis being $\pm 5 \%$.

It is assumed that the defect of cholesterol absorption by the mucosa of the gallbladder is the primary abnormality which causes excess cholesterol accumulation in the submucosa. Sequestered intracellular cholesterol obstructs bloodflow in these pseudopolyps creating avascular areas. These cholesterol "plaques" can enter the CBD, mimicking the symptomatology of choledocholithiasis by way of intermittent jaundice.

Only when obstructive lesions like cholesterol plaques in the gallbladder interfere with a normal periodic release of bile, intra-luminal cholesterol stones can develop due to stasis, especially when Rokitansky-Aschoff sinuses are formed in which stasis of inspissated bile constitutes a serious risk factor for stone formation. This can lead to a symptomatic, tense gallbladder which can be visualised radiographically as a hyperperistaltic "fighting gallbladder" associated with biliary collics (303).

In conclusion, cholesterosis is the primary factor whereby cholesterol stones and late manifestation of any obstructive intravesicular abnormalities for instance: septa, adenomyosis, cholesterol polyp etc., are secondary in nature. No common etiologic factor has been yet established. Certainly there is no relation to the production of hepatic lithogenic bile.

\subsection{Pancreatitis}

The close relationship between gallbladder, fiver and pancreas particularly in stone disease, is well established (205). The knowledge about pancreatic pathology became enriched with the use of ERCP and ultrasound (15). 
A common biliary-pancreatic channel, often seen on operative cholangiograms, predisposes to frequent occurrence of gallstone induced pancreatitis. Andersson (13) demonstrated the close correlation between patients with elevated serum pancreatic enzyme levels and the incidence of the anatomic communication between pancreatic and biliary ducts in man.

Under normal conditions the pancreatic duct pressure is higher than the distal CBD pressure. If reflux is observed in the pancreatic duct during operative cholangiography, in patients with normal pressure curves in the distal CBD, the main pancreatic duct can be visualised without sequelae of postoperative pancreatitis.

However, in high pressure states the "common channel theory" promotes proteolytic enzyme enhancement with bile contributing to a severe degree of parenchymal destruction of the pancreas. In those patients where the bile outflow resistance is increased pancreatic phospholipase-A can turn lecethin into active lysolecithin with known lytic effects on cells, thereby facilitating the development of infection. The stability of chalesterol will also change when the lecithin concentration is decreased by pancreatic enzyme activity because of a decreased micelle formation.

It has been suggested that pancreatitis and cholecystitis in stone disease have a common cause. In acute cholecystitis pancreatic enzymes have been determined in the gallbladder and can be considered of etiologic importance (312). Infection may spread via biliary lymphatic pathways. Patients with documented pre-existent biliary calculous disease with acute abdominal distress and elevated serum and urine amylase levels are often mis-diagnosed as having pancreatitis. The elevation of amylase levels are not organ specific. Especially in acute cholecystitis slight elevation of serum and urine amylase are frequently noted.

Pancreatitis may cause obstruction in the distal bile duct as has been shown by characteristic $X$-ray appearance $131 \%$.

This typical cholanglographic patterns of the distal CBD can be differentiated from primary biliary pathology.

Acosto (3) found gallstones in stools in over $80 \%$ of patients with pancreatitis within the first 10 days after the onset of the acute attack. In patients with associated pancreatitis the CBD can harbor in $20 \%$ calcull. This is approximately twice the incidence of stones in patients with gallstone disease but without the involvement
of the pancreas.

If pancreatitis is a complication of biliary disease the timing of the surgical intervention is extremely important.

This complication is mostly encountered in ampullary obstruction due to impacted stones. Any delay in the surgical treatment can detoriate the patients" condition because of progressive pancreatitis. Wide variations in the incidence of pancreatitis in cholelithiasis are reported. An average incidence of $15 \%$ seems justified. in chronic alcohol abuse, pancreatitis can occur in up to $50 \%$ of cases. If acute gallstone pancreatitis is first treated conservatively and the patient had to 
be operated upon because of a sudden development of acute abdomen, haemorrhagic necrosis, pseudocyst or abcess is not uncommon (3). These operative findings by Acosto suggest that the degree and duration of ampullary stenosis are important factors for the degree of severity which can not be managed conservatively.

Acosto's findings were not supported by others (286). Ranson reported (287) that pseudocyst formation and pancreatic abcesses are primarily related to relapsing alcoholic pancreatitis. The mortality rate for acute gallstone pancreatitis in the Acosto group was only $2 \%$, when patients were operated immediately, compared with a fatality of $16 \%$ if surgery was delayed.

However, early surgical treatment remains a controversy. Prospective randomised clinical trials have not yet been performed on early versus delayed surgical treatment. So far, a balanced medical and surgical treatment seems most advisable. Acute gallstone disease, complicated by mild pancreatitis frequently urge to a more aggressive approach in timing the surgical intervention. Recurrent bouts of pancreatitis during convalescence waiting for surgery should remind the surgeon to consider early operation.

The timing of surgery in the severly ill patient with acute gallstone pancreatitis and septicaemia should preceed first with resuscitative measures and administration of antibiotics (53). In general, there will be an improvement noticable after 24 hours of aggressive resustitative treatment. If surgery is planned after the patient is hemodynamically stable and the infection somehow under control, the morbidity of operative therapy will be decreased.

\subsection{Carcinoma of the biliary system}

The relation of carcinoma of the gallbladder and gallstone disease has been recognized for years. Histologic examination reveals almost predominantly adenocarcinoma. In a minority of the patients squamous cell carcinoma was found. Mesenchymal malignancies like sarcoma are very rare (300).

\section{Bile Duct Carcinoma:}

Biliary duct malignancies do not show a close relationship with stone disease. In inflammatory bowel disease (130), especially in ulcerative colitis, extensive hepato-biliary complications are frequently encountered. The time duration, severity, extent and involvement of the Gl tract seems to be well correlated with the severity of the associated liver disease (226).

The incidence of bile duct cancer is significantly increased in uicerative colitis and is reported to be several times higher than expected in the general population. Cholangiocarcinoma associated with IBD (Inflammatory Bowel Disease) also occur in a younger age group.

Patients with choledochocysts are at higher risk to develop a duct carcinoma.

Infected bile could play a role in carcinogenesis. Parasitic infection can also 
contribute to the development of a malignant lesion. In endemic chlonorchis infestation the incidence of bile duct carcinomas is high (182).

\section{Gallbladder carcinoma:}

Gallbladder carcinoma is the most common malignant lesion in the biliary tract and ranks fifth amongst carcinomas of the digestive tract. The incidence of gallbladder carcinoma is 2,5 per 100.000 . It is increasing over the last 40 years $(121)$.

Whether this increase is real or merely a reflection of a more accurate reporting of avallable statistical data is a matter to be settled. Extensive clinical data show that the association of gallbladder stones and cancer is sufficiently frequent to suggest some common factoris), although stones are not an obligatory finding in gallbladder cancer. A high cancer risk is involved with calcified gallbladders (50). It is emphasized that this disease is not a type of chronic cholecystitis but belongs to the cholecystosis group (Chapter 3.3).

Age prevelance in this type of cancer is $60-70$ years. Considering the older age group it is interesting to note that choledocholithiasis is almost never present in gallbladder carcinomas.

Coexistent CDS has been described by Perpetual (275) reporting one patient in a series of 75 patients with this type of malignancy.

On the contrary the incidence of gallbladder carcinoma in South-Western female Indians has been estimated to be 6 times of that of a non-Indian population (277).

This correspond to the increase of stone disease especially cholesterol calculi in this ethnic group.

The sex ratio in gallbladder carcinoma is reported to be $3: 1$ to $6: 1$ showing a distinct female predominance (409), whereby in a similar age group the sex distribution in cholecystolithiasis was found to be $1,5: 1$ female predominance. This also warrants precautious interpretation to suppose a close relationship between gallstone disease and cancer of the gallbladder.

In an extensive post-mortem study i 155\%, primary gallbladder carcinoma was encountered when a large number of intraluminal stones with chronic infection was present while the composition of the stones seemed to be of minor importance, whether pigment, mixed or pure cholesterol. However, pure pigment stones were almost never reported with cancer of the gallbladder. Wenckent 14091 found only in 5 patients of 4102 untreated gallstone patients gallbladder carcinoma discovered 19 years following radiological confirmation of cholelithiasis. This small number of $0.4 \%$ does not support the idea of prophylactic cholecystectomy for prevention of carcinomas.

However, the cancer risk in confirmed gallstone disease would at least accelerate the indication for early cholecystectomy (212). One should keep in mind the patient with chronic inflammation of the gallbladder supported by non-visualisation on oral cholecystography that early surgery cannot only be curative but preventive as well as far asgallbladder carcinoma is concerned. 
From the above mentioned (epidimiological) data there is yet no convincing evidence available in favor of a direct relationship between cholesterol stones and gallbladder carcinoma.

More than 50 years of aggressive cancer treatment did not change the cure rate of this dreadful disease. The tendency for local dissemination in the surrounding tissues are manifested late in the course of the dissease. Thus, detailed analysis of clinicall features for early cancer detection have limited value. The early cancer is still asymptomatic and the advanced one is unfortunately incurable.

The most favorable results have still been achieved by simple cholecystectomy in patients with small carcinomas predominantly histologically diagnosed postoperatively. The final outcome is poor regardless of the stage of the disease. Advances in radical therapy did not change the dismal outlook for patients with primary carcinoma of the gallbladder and 5 year survival rates have almost never been achieved. More extensive operations did not significantly prolong the patient's life, although a better palliation is claimed $(6)$. Recent success with radical surgery in locally extended carcinomas are encouraging (6), but warrants clinical trials. 


\section{CHAPTER 5}

\section{Medical stone dissolution}

Medical treatment of cholesterol gallstones by oral administration of cheno- and/or ursodeoxycholic acid is only successful in selected patients. Mostly it requires a minimum of six months to achieve gallstone dissolution (376). Even successful treatment of cholesterol gallstones in patients with coexistent CDS are reported $[24$, 3241.

Careful selection of gallstone patients remains a prerequisite for success in treatment of cheno medication (113).

These conditions are: 1. a patent cystic duct established on oral cholecystography:

2. radiolucent stones;

3. no history of severe (inflammatory) biliary disease.

Unfortunately many patients most in need of non-operative treatment do not easily respond to litholysis therapy (369).

These constitute all patients with longlasting gallbladder stone disease with severe infectious complications, with increased operative risks. On the contrary the asymptomatic patients with a large number of small floating stones on oral cholecystogram are best suited for medical therapy, but can be definitively treated by surgery with almost no complications at all.

Cholic Acid (CA), Chenodeoxycholic Acid (CDCA), and Ursodeoxycholic Acid (UDCA) have all been tested in clinical trials $(102,176,254)$.

$C A$ does not reduce cholesterol secretion in bile, nor reliably reduce saturation. Cheno improves bile saturation principally by lowering cholesterol secretion into the bile $(4,259)$. The effect of oral cheno medication on endogenous chenosynthesis is not yet settled. In patients on cheno therapy LCA increased to a maximum of $30 \%$ of total biliary $B A$ as inactivated, already sequestered $B A$ sulfates with no observed liver function defect.

CDCA is also inhibiting competitively CA absorption in the ileum. A combination of decreased synthesis and decreased intestinal uptake of $C A$ results in an absolute decrease in CA pool size in patients in cheno medication (176).

CA increases cholesterol uptake and CDCA has no effect or diminishes intestinal uptake. Thus CA may decrease cholesterol synthesis as a consequence of its effect on cholesterol absorption. However, this is of minor importance in the overall effect of $C A$ as a cholesterol gallstone dissolving agent.

UDCA, the $7-\beta$-isomer of CDCA, is not transformed by bacterial enzymes. Cheno and urso alike suppress cholesterol synthesis by reducing the HMG-CoA-reductase enzyme activity in the liver selectively $(236)$. Rowachol, an essential oil preparation. reduces the same rate limiting enzyme in cholesterol synthesis but is not yet widely
applicated as a stone dissolving agent (32).

In obese patients enzymatic activity of HMG-COA-reductase is increased as is biliary cholesterol output. Refractory results in obese patients can be achieved by nontherapeutic dosages. The average standard dose of CDCA and UCDA is obesity should thus be doubled or even tripled to obtain equivalent results. Weight reduction in severe obesity will naturally enhance the results of cheno 
medication by lowering endogenous cholesterol biliary output (238). The effective individuall CDCA or UDCA dose can be properly controlled by measuring the composition of duodenal bile. Depending on the degree of cholesterol saturation of bile, the oral dose can be corrected (186).

Success in treatment of BA medication is also determined by the total stone surface: one big stone takes longer to be dissolved than a large number of small stones. Stone volume may not exceed $50 \%$ of gallbladder volume (378).

The recommanded dose of CDCA varies from $0.5-1.5 \mathrm{mg} / \mathrm{kg} \mathrm{BW} /$ day in patients not exceeding $130 \%$ ideal weight (32). Individualized doses give the best results. The

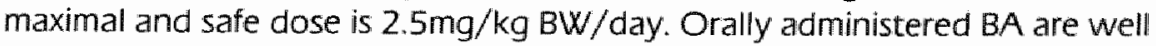
absorbed and efficiently mixed with endogenous BA in EHC. Thus, only organs involved in the EHC of BA are subjected to potentially toxic effects of $B A$.

Transient rise in liver enzyme levels are well established in CDCA treatment (33), but do not appear to be a problem in UCDA treatment. In liver biopsy specimen only mild histologic derangements have been observed.

There is no real explanation for this transient and selflimited liver damage (132), though the evidence favors lithocholic acid (which is formed from CDCA, but not from UCDA) being a known hepatotoxin. Identical liver changes in hepatocyte ultrastructures using EM in untreated gallstone disease has been established by Koch (203). However, no irreversible severe hepatotoxic reaction during BA medication has yet been recognized.

UCDA has many advantages over CDCA treatment. The therapeutic dose is much lower, being $150-600 \mathrm{mg} /$ day because urso is not dehydroxylated in the intestines and consequently replaces $C A$ and partly CDCA in the total BA pool. Significant side effects have not been published. Liver enzyme levels remains normal throughout treatment. Diarrhea, which is prominent during cheno medication, often limits the range of therapeutic doses because it is a dose related effect. The mechanism for the loose stool is almost certainly the effect of unabsorbed BA promoting water secretion from the colonic mucosa.

The increased BA load to the colon mucosa several years of CDCA feeding might provoke precancerous cell alterations. Colonoscopic control seems justified in these patients on cheno therapy to control this hazardous complication, which has not yet been attended to in clinical trials.

Adjunctive measures to increase results in treatment of litholytic therapy have been proclaimed to be low cholesterol intake and bran feeding (403). Phenobarbital decreases cholesterol secretion but did not potentiate the pharmacologic effect of CDCA and UDCA [235).

As previously explained also weight reduction, especially in severely obese patients, may enhance medical treatment.

Recurrence of stone disease can be prevented by continued cheno therapy after total disappearance of gallstones on oral cholecystograms. Perhaps intermittent prophylaxis would suffice $(272,377)$.

A possible favorable but as yet an unexplained side-effect of cheno administration is a significant lowering effect in hyperlipoproteinemia $(217,236)$ which has not been 
found in patients on urso medication. Urso, however, tends to raise HDL-cholesterol levels. Elevation of $\mathrm{HDL}$-cholesterol is thought to be perhaps beneficial in preventing atherosclerosis.

In conclusion, cheno therapy can be applied in only a small number of highly selected patients.

In particular, most patients with CDS do not fulffill selection criteria for litholysis therapy either, though a few successes have been reported for medical therapy of $\operatorname{CDS}(324)$.

Recurrence of stones is a problem after discontinuance of medication (377). In primary CDS such as in Caroli's disease cheno medication has been recommended following bilio-intestinal anastomosis with or without hepatic lobectomy to reduce future stone formation (207).

Reviewing pharmacologic proporties, UDCA is more effective than CDCA at lower doses, without apparent side-effects.

UDCA may eventually replace CDCA as the agent of choice for cholelitholysis. However, it is unfortunately also more expensive.

At present, insufficient data are available on the effects of these agents on plasma lipid metabolism and atherosclerosis, as well as possible delayed side-effects such as colonic carcinogenesis. Because of cost, and the dose-dependence of side-effects, a combination of CDCA and UDCA may eventually become the practical choice. 


\section{Part II}

Surgical considerations in gallstone disease 


\section{CHAPTER 6}

\section{Socio-Economic aspects of complications in Biliary Surgery}

Because of the high incidence of calculous biliary tract disease, cholecystectomy is the most frequently performed major abdominal surgical procedure today. Although cholelithiasis is predominantly a disease in the W/estern world, wide individual variations between countries still exist. The total number of cholecystectomies per one million of inhabitants is reviewed in table 6.1.

Tabele 6.1

Number of cholecystectomies per million inhabitants/annum

$\begin{array}{ll}\text { U.S.A* } & : 3.500 / 1 \text { mill. } \\ \text { Sweden } & : 5.000 / 1 \text { mill. } \\ \text { Netherlands*** } & : 1.400 / 1 \text { mill. }\end{array}$

* : F. Glenn, IBA Congress Report 1980, Maastricht

** :S. Bengmark, Ann. Surg. 189: 447.1979

*** : See Table 6.2

The total cost of disability, medical care and hospitalisation combined with the temporary inability to continue the daily activities arising from sequelae of complications, places the disease and its treatment in the focus of any health delivery care system.

In case of a medical stone dissolution treatment, a high recurrence rate can be expected after discontinuation of the admininstration of the drug. The life long drug dependency cannot be assessed financially at this stage but we will reach astronomical figures. Apart from the financial aspect the final evaluation of this type of treatment is not yet available, when given on a long term regimen.

Table 6.2

Total number of Gallstone Operations in the Netherlands during 1975-1979*

\begin{tabular}{lccccc}
\hline & 1975 & 1976 & 1977 & 1978 & 1979 \\
& & & & & \\
1. Cholecystectomy & 21311 & 20736 & 21242 & 20305 & 19316 \\
2. Emergency cholecystectomy & 1226 & 1296 & 1300 & 1152 & 1164 \\
3. CDE (\% of total number & 4663 & 4564 & 4722 & 4463 & 4109 \\
cholecystectomies) & $(18.0 \%)$ & $(18.0 \%)$ & $(18.2 \%)$ & $(18.0 \%)$ & $(17.5 \%)$ \\
4. Re-explorations $(\%$ of & 242 & 292 & 275 & 201 & 125 \\
total number of CDE) & $(5.2 \%)$ & $(6.5 \%)$ & $(5.8 \%)$ & $(4.5 \%)$ & $(3.0 \%)$ \\
5. Bilio-digestive anastomosis & & & & & \\
prim. procedure & 225 & 235 & 419 & 539 & 583 \\
sec. procedure & 97 & 127 & 79 & 53 & 12 \\
total & 322 & 362 & 498 & 592 & 595
\end{tabular}

* Stichting Medische Registratie/Utrecht (1981) 
Common Duct Exploration /CDE/ increases the morbidity in cholelithiasis. In the Netherlands the total number of re-explorations is steadily decreasing probably due to the more frequent selection of a bypass procedure during the primary CDE Table 6.2).

Re-exploration for retained stones is a heavy burden on hospital costs (Table 6.3).

Table 6.3:

Cost of treatment of Retained Stones per patient

Hospitalisation fee (14 days):

Diagnostic work-up :

Doctors fee land extra charges:

Total:
$\$ 3.150$.

$\$ 1.500$.

$\$ 750$.

$\$ 5.400$

The total costs pro patient with retained stones in the Netherlands can be estimated between $f 13.000,-$ and $f 20.000,-\$ 6.000,-1$. An estimated 300 patients underwent reexploration accounting for $\mathrm{fl} .5 .10^{\circ}$ in 1976 alone. The comparative data for the USA and Sweden, considering the total number of cholecystectomies performed (see Table 6.2) are $\pm \$ 80.10^{6}$ and $\$ \$ 4,5.10^{6}$ respectively.

The ever increasing health care cost prompts us to reconsider and reevaluate our treatment modalities to find ways and means to improve patient care without increasing or hopefully decreasing the costs.

Because most complications in biliary surgery are caused by retained stones, a reduction of residual stones postoperatively will not only lower in morbidity and mortality but the related expenses as well.

Technical improvements can still be made by accepting routinely radiologic studies and an uniform lithotomy including endoscopy in every patient as will be shown in this study. 


\section{Chapter 7}

\section{Common Duct Stones}

\subsection{Introduction}

Common Duct Stones (CDS) are encountered in approximately $7-15 \%$ of all patients with gallbladder disease $(264,265)$.

CDS are found in approximately $60 \%$ of all CDE performed. Increasing the accuracy of operative cholanglography the percentage (unnecessary) negative biliary explorations can be reduced.

In the elderly age coexistent CDS are more frequently established during cholecystectomy and the female predominance is less pronounced. The overall female/male ratio in cholelithiasis is about 3:1. This is primarily true up to the menopausal age. From this age the ratio of females to males is about 1.5:1. In general, with a long history of the disease, CDS is very likely to develop simultaneously regardless of the age of the patient(255).

Acute cholecystitis is a disease of the older age group and occurs more frequently in patients over 65 . This contributes for the high proportion of CDS in patients with acute cholecystitis, which is reported to be $19-25 \%(78,403)$. Especially when acute cholecystitis is accompanied with high serum bilirubine levels, coexistent CDS can be expected. Watkins (403) demonstrated in his series an incidence of jaundice in patients with cholecystitis being $73 \%$. In non-jaundice patients with acute cholecystitis in only $6.5 \%$ billary calculi were found during early operation. Glenn (142) however, reported a $9.5 \%$ incidence of coexistent CDS in patients presenting with acute cholecystitis. His series covered a period of 46 years experience in biliary surgery, but he did not mention the routine use of operative cholangiography which may be responsible for this relative low figure.

Suggestive evidence on CDS is frequently lacking preoperatively and consequently a high percentage of unsuspected CDS is diagnosed by operative cholangiography if it is performed properly and routinely. Berci 1411 reported a $4-10 \%$ incidence.

Most patients with CDS are symptomfree. Unlike in the urinary tract there are no muscle fibers in the common duct wall responsible for rythmic contractions. That is why biliary colics correspond to stone passage through the papilla or to gallbladder contraction but never to $C B D$ stones. These silent stones are frequently discovered at autopsy with no severe accompanying pathology like secondary biliary cirrhosis or cholangititis (247).

In the non-jaundiced patients without cholecystitis single elevation of $\mathrm{g}$-Gt $15 \mathrm{~g}$ Glutamyl-transpherase). 5-N (5-Nucleotidase) and APh (Alkaline Phosphatase) is seldom pathognomonic for biliary stones. Ultrasound can be very helpful in the patients with biliary tract obstruction when other radiological examinations would fail (37). In a non-obstructed CBD, the diagnosis of biliary stones remains difficult. The width of the CBD can be assessed but constitutes only an indirect parameter because even a non-dilated CBD can harbour small stones (151).

Even in those cases with no specific history, normal liver function tests, nor any supporting radiologic appearance [CT-scan, Ultra Sound], stones in the CBD should 
be expected. This is one of the reasons to proclaim the necessity of routine operative cholangiography in every "simple"cholecystectomy.

\subsection{Etiology}

Cholesterol is the main component in CDS. So far no significant difference in chemical composition between gallbladder stones and CDS has yet been demonstrated.

Cholesterol micro-crystals are frequently found in the aspirated bile from the duodenum and can be indicative for patients at risk to harbor gallstones. Microcrystals have never been found as a nucleus. Analysis of a nucleus is seldom carried out but it would seem to be of importance from the etiologic point of view on CDS |366|.

Only Been (28) has analysed the nucleus of typical cholesterol stones. The inside contained hydroxy-apatite with high concentration of sulphur, magnesium and iron. The outer layers were composed of cholesterol and proteins.

Nucleation is the primary event and cholesterol micro-crystals will precipitate on freshly formed nuclei initiating the growth of the stone. The physico-chemical reactions in bile production and composition are responsible for a continued process of enlargement of stones (58).

Inorganic substances can also act as a nidus. This has been demonstrated by Silvennoinen (343) both experimentally and in man. Silk suture material used for ligating the cystic duct was found.

This phenomenon has been also demonstrated by Hanemaayer (160).

Amorphous material derived from cholecystostomycatheters can also readily pass into the CBD. Glenn $139 \%$ found in 26 instances in a total of 43 secondary explarations amorphous material in the CBD following cholecystostomy. This was suggested to be related to a foreign body response triggered by the catheter. Indwelling T-tubes also can induce stone-formation. Biliary sludge is predominantly composed of unconjugated bilirubine. This material may also provide a nidus for stone formation in cholesterol stones, especially in case of stasis.

Pancreatic reflux in the biliary system should be considered as an additional etiologic factor in stone formation (chapter 3.3.).

In biliary obstruction special pathophysiologic phenomena on bile acid and cholesterol metabolism are noteworthy indicating a progressive course in stone formation once biliary stones are already present.

Cholesterol synthesis in the liver as well as cholesterol secretion is accelerated in case of biliary obstruction (chapter 2.11 ). Consequently a rise in biliary cholesterol concentration increases the lithogenicity. Any bile flow disturbance will thus increase the risk for stone formation. This should be kept in mind in dealing with impaired bile flow in the presence of CDS.

\subsection{Pigment stones}

Pigment stone pathogenesis involves bile pigment metabolism. It has to be 46 
differentiated from cholesterol stones, because pathogenesis and treatment are quite different.

Demographic differences with cholesterol stone disease are pronounced. In Asia, the pigment stone is the most common variety and appears to be associated with bacterial $(151,214)$ and also with helmintic infections $(67,237)$. Hemolytic disorders are associated with a high prevelance of pigment stone disease such as: Haemolytic Spherocytosis, Thalassaemia and Sickle Cell Disease.

Rheumatic heart valve disease and patients with a ball-valve prosthesis show an incidence of $39.1 \%$ of gallstone disease 1161$)$. These findings suggest that gallbladder stones could be a late complication in patients with arterial heart-valves. The underlying mechanism is an excess of free pigment resulting in an increased hepatic secretion of bilirubin from the rapid haemoglobine breakdown.

Table 7.1

Distinct differences in pigment and cholesterol stone disease

Pigment Cholesterol

\section{Epidemiology}

- Demography

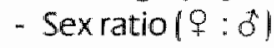

\section{Etiology}

- diet influence

- obesity

- hormone influence

Disease ass. pathology

- biliary infection

- cDS

- cancer

\section{Surgical treatment}

- cholecystectomy

- bypass surgery

- non invasive techniques

\section{Oriental \\ No striking female \\ predominance}

1. Microbiologic *

2. Haemolytic **

$-$

$-$

$-$

prim.

prim.

H

symptomatic procedure primary

never
Western World

$4: 1$

Biochemical

+
+
+

sec.

sec.

$\pm / ?$

* : Parasitic as well as bacteriologic

**: see text. 
Bacterial enzymes are known to induce deconjugation in the biliary tract. W/ith adequate amounts of calcium ions in excess, deconjugated bilirubin precipitates in the biliary passages.

In vitro-lests have shown that $\beta$-glucuronidase, secreted by Escherichia Coli, increases hydrolization of bilirubin to a water-insoluble form resulting in precipitation of calcium bilirubinate.

In parasitic infestation of the biliary tree the roundworm, mainly Ascaris. Lumbricoides, provides a potential agent for a nidus (325).

The geographic distribution of pigment stone disease, is characteristic. in Japan the incidence of pigment stones has declined following the decrease of roundworm infections to almost $1 \%$. Also changes in the diet since the end of World War II has contributed to the increase of cholesterol gallstones in Japan.

Chainof (81) found a very high osmolarity of bile in patients with pigment stones stimulating the formation of biliary mud in the radicles. This may be of additional importance in pigment stone disease.

Complications of the gallbladder with pigment stones are less frequent and are seldom reported to give fistulae, empyaema, Mirizzi gallbladder etc., which are common in cholesterol stone disease. The clinical picture in pigment stones is dominated by complications in the extrahepatic biliary system like cholangitis and septicaemia (142). In summary: Pigment stones have a distinct pathogenesis in which the biliary complications prevail.

In cholesterol stone disease however, the gallbladder stones are responsible for almost every complication and predict the clinical outcome.

Consequently, the therapeutic surgical strategy is different (Table 7.1.) in both disease entities.

\subsection{Primary Common Duct Stones}

Primary common bile duct stones, recurrent or reformed, constitute al single clinical entity.

The hypothesis is that Common Duct Stones (CDS) are predominantly secondary. having migrated through the cystic duct into the Common Bile Duct (CBD). The lack of physiologic and chemical data on stone formation further hinders accurate discussion. The pathophysiologic role of bile composition in stone disease have still remained speculative as far as reformed stones are concened (196).

Madden (230) emphasised the macroscopic appearence of calcull as the most reliable parameter to differentiate between primary and secondary CDS. He found in I out of 5 patients with CDS an empty gallbladder. This relatively high percentage was not supported by others. Histology of the gallbladder wall was not mentioned by Madden, which could have shown the existence of a long-standing gallstone disease. Even the chemical bille composition was not established, to differentiate primary from secondary stones. Neither the function of the sphincter was considered to be of any etiologic importance in this study. 
The clinical criteria to define true primary CDS (306) are:

- status post-cholecystectomy

- at least a 2 year asymptomatic period

- soft earthy appearence of stones

- no outflow obstruction (sphincter)

- absence of long cystic duct remnant.

Uising these criteria Cameron reported a $4 \%$ incidence, contrasting to data published by Madden who presents a $50 \%$ incidence on primary CDS in all patients with stones in the $C B D$.

Braash (67) reported his experience which showed that only during the primary explaration in $50 \%$ of these type of cases operative cholangiography was performed, and only few patients underwent operative biliary endoscopy. Papillary fibrosis was encountered in 1 out of 67 patients with primary CDS (306). Schein has demonstrated that this phenomenon can be the consequence of instrumental damage or spontaneous passage of stones resulting in an injury to the papilla. This can result in an increased resistance due to fibrosis, and as at consequence stasis or impaired flow leading to formation of primary stones. Contributing factors for CDS are: benign strictures, sclerosing cholangitis, polycystic disease and Caroli's disease (282).

Treatment in patients suspected of primary CDS is controversial. This is because insufficient data are available yet on the natural history of primary CDS to recommend a biliary enteric anastomosis at the initial operation.

Saharia (306) followed a group of patients with primary CDS. A symptomfree interval for more than 5 years was found in over $80 \%$. This clinical observation on primary CDS would indicate that in case of exploration simple removal of stone(s) and checking simultaneously the sphincter function would eliminate the necessity of a bypass procedure.

in conclusion, the labelling of CDS into primary or secondary stones is still a semanticism until more convincing data are available (chapter (4).

\subsection{Retained stones}

Retained bile duct stone(s) (RS) continues to be a problem despite advances in surgical techniques. The presently reported incidence do not differ considerably from the number of RS seen 40 years ago. The variation of figures published on this topic is mainly due to the differences in interpretation and mathematical acrobatics. The incidence of RS should be assessed in the following way:

Total No. RS $\times 100 \%$ No. pos. CDE

This ratio only accounts for the direct postoperative RS discovered immediately postoperatively by way of T-tube cholangiography.

The exact number of RS is suspected to be even higher because biliary stones 
appearing in the late postoperative phase are disregarded. Besides, these stones are more likely to be classified as reformed rather than retained stones because symptoms appear only years following cholecystectomy.

Reviewing a number of publications, RS was sometimes calculated to the total number of cholecystectomies which is wrong because it resulted in a much lower incidence. It should be related to the number of (positive) CBD explorations. Even though it is uncertain how many cases from the group of a "simple" cholecystectomy with undetected and unsuspected stones will after years of a symptomfree episode come to a secondary exploration.

Table 7.2

Literature review on Retained Stones percentages

\begin{tabular}{lccccc}
\multicolumn{1}{c}{ Author } & $\begin{array}{c}\text { Cholecys- } \\
\text { tectomies }\end{array}$ & CDE & $\begin{array}{c}\text { Positive } \\
\text { CDE }\end{array}$ & $\begin{array}{c}\text { Retained } \\
\text { Stones }\end{array}$ & $\begin{array}{c}\text { Ret. } \\
\text { Stones \% }\end{array}$ \\
\hline Glenn $(1974)^{*}$ & 16201 & 4004 & 1837 & 174 & 9.4 \\
Glenn $(1968)^{+}$ & 2395 & 658 & 300 & 24 & 8.0 \\
Glenn $(1974)^{++}$ & 499 & 83 & 47 & 4 & 8.5 \\
Sappala $(1977)$ & 1483 & - & 188 & 17 & 9.0 \\
Smith $(1957)$ & 1381 & 224 & 123 & 15 & 12.2 \\
Corlette $(1972)$ & - & - & 100 & 22 & 22. \\
Bergdahl $(1976)$ & 4078 & 972 & 512 & 69 & 13.5 \\
Smith $(1968)$ & - & 316 & 166 & 23 & 13.9
\end{tabular}

* : Collective data, concerning series from '43- 89 , none of which is separately reported in this table;

* : Biliary surgery in New York Hospital-Cornell Medical Center (NYHCMC during 1950-1966:)

: Biliary surgery NYH-CMC 1972;

see text for legend:

In Table 7.2 the RS percentages are summarized and recalculated according to the above mentioned formula. Apart from the series by Corlette (91) others reporting an average of $10 \%(8.0-13.9)$, or in a collective series $(200)$ a wide range of $7-28 \%$ is recorded.

RS are more likely to occur in patients in whom CDE was positive rather than in patients following simple cholecystectomy (350). This warrants special attention when dealing with CDE for stone extraction. The CDE is incomplete if an initial operative cholangiography has not been performed. Completion cholangiography reveals almost in one third of all positive CDE a retained stone(s) (405). In cases of secondary explorations the incidence of overlooked stones is even higher 1601 . A final operative cholangiography is the method of choice in combination with endoscopy in equivocal cases.

However, in most series cholangiography failed to demonstrate RS for different reasons (Table 9.1). The failure rate of operative cholangiography approximately 
equals the incidence of $\mathrm{RS}_{x}$ but is sometimes enhanced by difficult interpretation following instrumentation especially in the distal CBD.

The incidence of unsuspected stones is in average $5 \%$ in all cholecystectomies where clinically no indications are present to explore the CBD. Spontaneous passage of stones is unpredictable. Bergdahl [47], reported $50 \%$ of RS disappeared within 30 days postoperatively. In his retrospective series on RS management most patients $128 / 401$ were reoperated within that period of 30 days, so stone passage could be expected to be higher if reoperation was postponed Berci and Hamlin /41/ reported a $9 \%$ stone disappearance during a waiting period of 4-6 weeks.

The problems of recurrent stones to determine whether they are overlooked at the primary operation or they are reformed, are still unanswered:

- are stones missed at CBD exploration?

- are stones left behind after cholecystectomy?

- are stones reformed because of stasis due to outflow disturbance?

- are stones reforming because of changes in the bile composition?

These above questions need to be answered.

The composition of true recurrent stones is claimed to be pure calcium bilirubinate. Continued supersaturation of bile in the cholecystectomized patient could then only be of minor importance etiologically|379|.

The following surgical-anatomical problems can play a factor for R.S:

1. intra-hepatic gallbladider $(214)$;

2. a long cystic duct remnant (41);

3. amputation of gallbladder.

Stones may be left in a retained Hartmann's pouch or in the long dilated cystic duct remnant. A stone hidden in a cystic duct remnant can escape detection on the completion T-tube cholangiogram. While an intra-hepatic gallbladder is a rare anomaly, a long remaining cystic duct and even incomplete gallbladder resection is frequently found after biliary surgery.

RS is a serious clinical problem with an increased morbidity, mortality and extended hospital stay. Even in cases of bilio-enteric bypass residual stones can cause complication at the anastomic site (52). The degree and extent of complications due to RS is unpredictable (139). The problem of RS cannot be solved by performing CDE regularly following every cholecystectomy because of the increased morbidity and high number of negative explorations (139).

The risk of operative injury in the ductal system is high in performing a choledochotomy in a normal sized CBD even if stone(s) are present.

Corlette $(91)$ reviewed operative cholanglograms in 100 consequetive CDE with a RS incidence of $22 \%$. Retrospectively the operative cholangiograms did show the missed stones in $75 \%$ (") of the patients even though many of the cholangiograms where of poor quality The substandard results of operative cholangiography and the time consuming aspect are the main reasons why surgeons are still skeptical or reluctant to employ it and rather rely on surgical exploration techniques $(91,1391$. A closer cooperation and mutual understanding of the problem between the radiologist and surgeon would easily lead to a more satisfactory result concluding in 
a reduction of overlooked stones $14 \mathrm{l}$. The burden of this complication in a benign curable disease would be almost eliminated if these constant repetitive shortcomings would be understood by bothparties (Chapter 9.2). 


\section{Chapter 8}

\section{Common Bile Duct}

A brief review on benign bile duct pathology is presented with special emphasis on biliary endoscopy.

\subsection{Surgical anatomy}

The biliary system of surgical importance is mainly the extra-hepatic system (Fig. 8.1 ). The anatomy of the intra-hepatic radicals and intra-lobular distribution of bile ducts is of interest in case of segmentectomies or lobectomies in liver surgery.

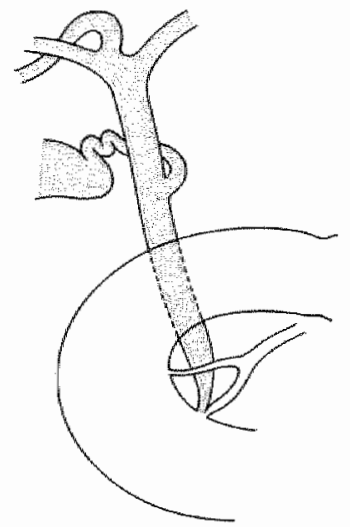

fig. 8.1: Extra-hepatic biliary system

The extrahepatic system is composed of:

- left and right hepatic ducts (LHD and RHD):

- Common Heparic Duct (CHD):

- Common Bile Duct (CBD).

Only a short segment of the right hepatic duct, including the dorsocaudal branching, is considered extrahepatic. The extraparenchymal LHD can easily distend in case of obstruction, resulting therefore in a larger diameter than the RHD.

During cholangioscopy the dorso-caudal duct can sometimes be seen originating directly from the bifurcation, forming the trifurcation. A number of anatomic variations in the entire biliary system has been extensively documented by Wiechel (41). The largest variety of type in drainage and configuration is presented by the cystic duct. The junction can arise anywhere from the RHD to the ampulla (Fig. 8.2) Consequently, wide individual variations in length of $C H D$ and $C B D$ are noted. The cystic and the bile duct can join laterally, posteriorly or anteriorly, entering proximally into the ampulla or into the CBD. This is in contrast to the statements on biliary anatomy in most surgical and radiological textbooks.

Cystic duct agnesia has been erroneously described in patients with a gallbladder in situ. This should be regarded as an acquired rather than a congenital variation: in a chronic inflamed, distended gallbladder the cystic duct can be very short. 
A severe infection in Calot's triangle initiated by a stone erosion in the cystic duct has been described by Mirizzi, forming a fistula between CHD and Hartmann's pouch, resulting in dislodging gallstones into the CBD with obstruction (250).

\subsection{Width of Common Bile Duct}

The CBD consists mainly of elastic fibers. Sparcely distributed smooth muscle fibers have been identified. Only in the region of the papilla does the CBD las well as the pancreatic duct) have semi-circular, oval shaped muscular layers. These sphincters function independently $(65,181)$. Duodenal peristalsis has no effect on the sphincter (99).

The $C B D$ widens with increasing age. An approximate formula relating the internal diameter to age is:

$\frac{15 \times \text { age }}{100}=$ width in $\mathrm{mm}$.

It has been suggested that the diameter of the CBD may increase in width following cholecystectomy in men. Oddi stated that this was a normal post-cholecystectomy phenomenon (422).

Cruder reported on patients with a non-functioning gallbladder. He found the width of the CBD was not different from the CBD in patients following cholecystectomy (156)

After spontaneous passage of stones a temporary CBD dilatation can be expected which has been experimentally confirmed in dogs by Berci $(42,43)$.

\subsection{Physiology of sphincter mechanism}

To establish a proper physiologic equilibrium a para- and orthosympathic innervation has to be present (209). This can easily be demonstrated by pressure gradients in the extra-hepatic biliary system obtained by manometry, after stimulating the different nerves independently (376).

Differences in anatomic pathways of both orthosympathetic and parasympathetic systems are illustrated in Figs. 8.2 and 8.3.

All abdominal viscera are innervated through the sympathetic celiac ganglion via post-ganglionic adrenergic fibres, stimulating:

1. smooth muscle:

2. secretion:

3. vasoconstriction:

4. visceral pain perception.

Except for visceral pain perception, all functions are conducted by efferent fibers. Blocking the sympathetic ganglion with $1 \%$ Procaine, Schein (316) was able to show an increase in pain perception by elevating the intraductal pressure to $50-60 \mathrm{~cm}$ $\mathrm{H}_{2} \mathrm{O}$, which is 5 to 6 times higher than the average passage pressure of the sphincter measured intra-operatively. 


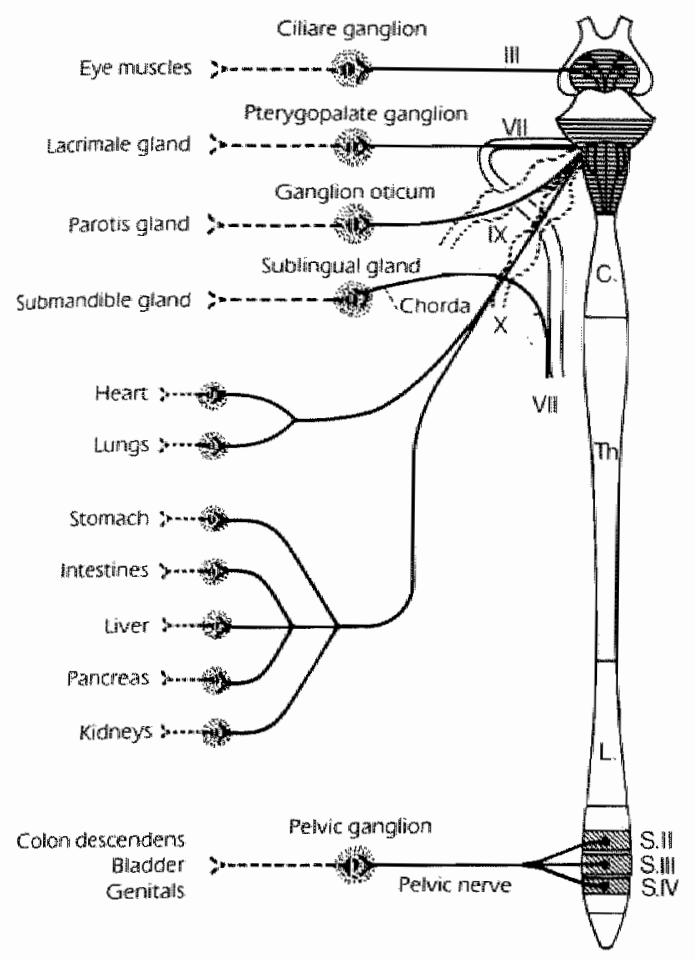

fig. 8.3

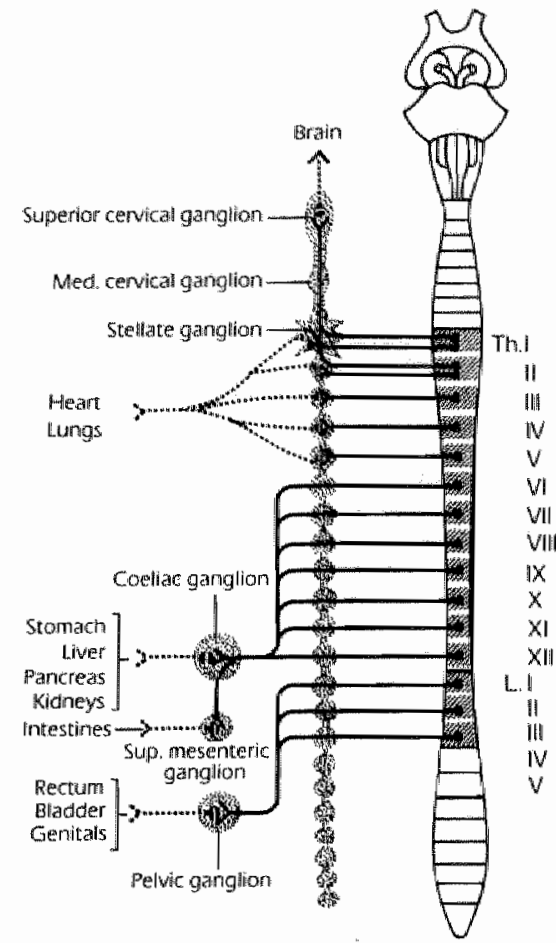

fig. 8.2

A study by Dardik (104, 105) determining the presence of biliary $\alpha$-and/or $\beta$ receptors was performed using receptor specific stimulating and blocking pharmacologic agents. Administration of DHE (dihydroergotamin) which blocks areceptors, has no effect on the biliary sphincters. Lechin (213) however, did find $\alpha$ receptors in the gallbladder wall, because CCK mediated contraction could be antagonized with DHE. The balance between adrenergic and cholinergic innervation of the biliary sphincters change in favor of the sympathetic system following vagotomy resulting in a relaxation of the sphincter.

The initial fall in pressure after truncal vagotomy by a decreased sphincter tone is on average $35 \%$ (315). It can be restored to normal values by administering pilocarpine, a parasympathicomimetic drug.

Administration of atropine can not further reduce the already low pressure following vagotomy. Neither a change in the hepatic arterial or portal blood flow could be demonstrated by these experiments.

Even bile flow nor the diameter of the CBD was affected by vagotomy. The results of hepatic vagectomy both in experimental and clinical studies, have shown a significant decrease in sphincter resistance. The hepatic vagectomy should have some rational but would require more clinical data to become widely accepted in selected cases. 


\subsection{Pathology of Common Bile Duct}

Infection:

A histologic study on CBD wall in cholangitis was performed by Schein (318). Beneath the epithelium multiple glands can be identified. In cholangitis these glands are inflamed and develop a peri-glandular edema which can be seen through the cholangloscope. This finding was confirmed by Juvara (194)

In case of chronic infection due to hyperplasila and hypertrophy of these glands, mucoid substance is secreted, simulating a purulent discharge, which is responsible for the coagulum-like material in cholangitis and which is seen around stones (fig. 8.3).

The epithelium never displays severe ulceration, necrosis or abcess formation. The dominant acute infectious reaction is a subendothelial congestion and oedema with hypertrophy of the glands. These histologic findings justify a safe bilio-enterlic anastomosis even in the presence of severe cholangitis.

Primary sclerosing cholangitis is a rare condition (2). Sometimes it is associated with ulcerative colitis (55) or retroperitoneal fibrosis (233). The sclerosing process usually affects both intra-and extra- hepatic ducts. A segmental sclerosis has seldom been reported and should be differentiated from a sclerosing carcinoma of the bile duct. Histologic appearance of the bile duct in primary sclerosing cholangitis show extensive fibrosis with an intact epithelium which can be biopsied with the help of the cholangioscope (221). Bile stasis is enhanced by the multiple strictures which may sometimes lead to secondary stone formation (Chapter 7.4). The unfavourable prognosis is determined by a progressive fibrosis ultimately resulting in liver failure and death. Treatment by intra-hepatic enteric anastomosis is only symptomatic but has no influence on the progress of the underlying disease.

\section{Pancreas related pathology.}

The pancreas is originally constructed of two lobes. During the embryologic stage the duodenum enlarges, enforcing external rotation and lateral displacement towards the retroperitoneum (181). The proximal part forms the cauda. This drains separately via a minor papilla in $85 \%$ and is known as Santorini's duct. The duct of Wirsung drains the head of the pancreas and communicates with the ductal system of the cauda forming the main pancreatic duct. However, the functional significance of the remaining duct of Santorini is seriously questioned. In distal obstruction of the $C B D$, no retrograde pancreatic or biliary drainage into the duodenum via the minor papilla has been confirmed.

Parenchymal pathology in the head of the pancreas has serious implications on bile flow. The appearance or "slope" of the distal CBD on cholangiograms can give us some hints about the involvement of the pancreas $(159,311)$ to differentiate between primary biliary or pancreatic pathology. 
Congenital anomalies.

The most frequently reported benign abnormality of the extra-hepatic billiary system is a choledochocyst $(145,423)$. It can be solitary or multiple (100) distributed alongside the entire CBD and CHD. It can cause intermittent and/or incomplete biliary obstruction. Miedener (246) reported a patient with isollated elevated g-GT serum levels and epigastric discomfort caused by a choledochocele protruding intermittently into the duodenum. This entity was treated by endoscopic retrograde coagulation. The patient became symptomfree.

The increased incidence of malignant degeneration in congenital cysts is well recognized [423]. Experience with internal drainage by way of a cysto-enteric anastomosis has not been very encouraging because of recurrent infection and narrowing of the anastomoses. Therefore a resection is favoured. Congenital celes are also reported in the gallbladder (12).

A rare cholecystocele was reported by Kihne (201). It bypassed the atretic CHD to the distal CBD via the cystic duct.

Intrinsic bile duct anomalies may accompany congenital duodenal deformaties. Acute cholecystitis with stones was frequently noted by Tchirkow (373) in children several years following surgical correction of the duodenal abnormality. An intraoperative transhepatic cysto-cholangiogram is advisable following the duodenal correction to exclude coexistent congenital biliary atresia.

Tumors.

Mucine producing glands are distributed through the entire bile duct.

Tumours, multiple or single (260) are derived from these mucoid glands and are known as papillary adenomatosis. This disease can follow a clinical malignant course with dissemination throughout the biliary ducts.

Biliary papillary adenomatosis is considered by most authors $(67,188)$ as a benign disease.

Helpap (168) demonstrated a malignant course in a patient with rapidly progressive recurrent intra-hepatic papillomatosis which developed into a papillary adenocarcinoma.

A single papiliary adenoma in the gallbladder or the bile duct has a favourable prognosis but intra-hepatic multiple papillomatosis should seriously be regarded as a pre-malignant condition. 


\section{Chapter 9}

\section{Common Duct Exploration}

\subsection{Introduction}

The mast frequent indication to explore the Common Bile Duct is stone disease. Foreign bodies (409), parasitic disease or a benign growth are very uncommon. The indication to perform a CDE is supported by operative cholangiography. Manometry and flow measurements are additive techniques in operative diagnostics. The reliability of the clinical criteria to predict stones is small: a dilated duct correlates only in $50 \%$.

A dilated cystic duct can promote CDS in 35\%. Even palpation can be misleading, especially in the hepatic (intraparenchymall part of the biliary system. Also the distal duct, when induration of the pancreas head is present like in chronic pancreatitis. cannot be reliably palpated.

\subsection{Flow and pressure measurements}

The first studies on manometry (41) were independently performed by Caroli, Mallet-Guy and Hess (1946-1948). It is a simple technique to monitor sphincter dymamics. This mechanism operates with low pressure gradients: $10-20 \mathrm{~cm} \mathrm{H}_{2} \mathrm{O}$. That is why manometry should be performed with care to exclude misinterpretations. Proper maintenance of the equipment is essential. Through the cannula both pressure and flow can be measured. When introduced into the cystic duct artefacts (cannula against the CBD wall) can impede flow or increase pressure, resulting in unnecessary biliary explorations.

The reported fallure rate of cystic duct cannulation in different series is 10-15\% 157, 240) and mostly concerns the larger Champeau cannula. In cases of secondary exploration the CBD has to be punctured with a needle or cannula, which has to be kept or secured in position to avoid leakage. This can be achieved with a special puncture-clamp designed by Berci (fig. 9.1).
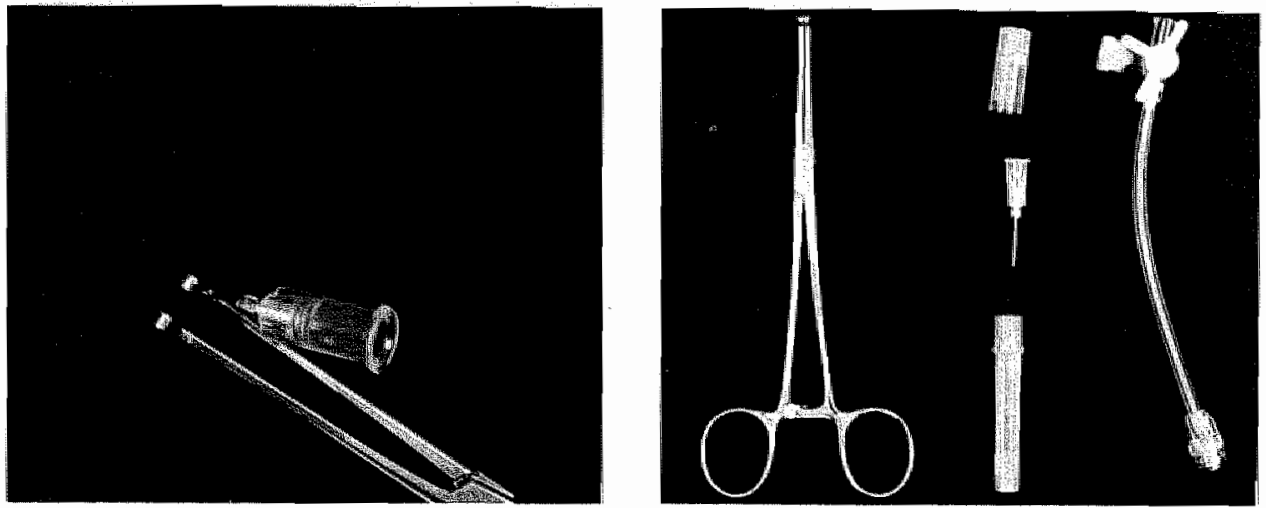

fig. 9.1: Modified Abbott Clamp for puncture cholangiography (Sulgi-Aid inc., North Hollywood, CA. 91605 ) 
Different "normal values" of manometry are reported. If it is used in conjuction with chalangiography, the pressure should be corrected with the specific gravity of the contrast material. In the original report by Mallet-Guy the normal value of residual pressure was $15 \mathrm{~cm} \mathrm{H}_{2} \mathrm{O}$. Schein considers the upper limit of the passage pressure: $20 \mathrm{~cm} \mathrm{H}_{2} \mathrm{O}$ above which sphincter pathology can be suspected (61). All measured data (initial, passage, resting or residual pressures) should be considered rather than to rely on one single standard pressure value. False positive results can be ruled out easily by using all available pressure data (206) as a guideline.

De Boer (57) reported a series of 279 patients which underwent CDE. In 148 patients CDE was negative: In only 22 patients $(7,9 \%)$ a false positive result was obtained. In 63 patients with biliary stones pressure was normal resulting in a false negative percentage of examination of $22.6 \%$. In his experience in stone disease manometry was of no value in approximately $1 / 3$ of the cases.

Schein (314) does not analyze failure rates in his different groups of patients. In 19 patients with acute cholecystitis an increase in biliary pressure was found without the presence of stones in the CBD. This high pressure may represent ampullary or cholangitic reaction which in some patients correlated with the high serum bilirubin levels. Manometric documentation appears to be a reliable aid to the diagnosis of ampullary fibrosis.

In secondary exploration manometry combined with radiology is of additional value because papillary fibrosis is probably the result of previous injury (instrumental- or spontaneous passage of stones) (371).

If cholangiography was performed without manometry stones should have been missed by $10 \%$ of all patients undergoing cholecystectomy (240). If the diameter of the stones are smaller than $4 \mathrm{~mm}$ they may pass spontaneously. Consequently this diameter serves as the minimum in stone detection in cholangiography.

Some studies advocate an accuracy rate for operative radiography as high as 88 $95 \%(46,281)$ which is related to the film quality (information-available) and interpretation. This can be further improved with manometry and flow measurement to be used in conjunction with cholangiography.

The accuracy rate of combined techniques [cholangiography and manometry] is greater than with either test by itself $|499,372|$.

However, both manometry and flow measurements are not suitable to detect stones located in the proximal or intrahepatic ductal system (179).

In summary, manometry has been proved to be an indispensible technique in research on biliary dynamics (314). The clinical value is limited to secondary explorations especially to establish the function of the sphincter (101).

In general the false negative percentage of manometry is higher than in case of flow measurement, although the reported accuracy rates of both techniques are less related to modern fluorocholangiography.

\subsection{Operative cholangiography}

After 50 years following its introduction, this subject is still a controversy. The major drawbacks are: it is time consuming, the percentage of non-informative films can be 
high due to technical errors or poor performance of these small mobile $x$-ray machines and least but not last in some critical cases, a consulting radiologist is not availabile.

Complications are seldom reported: an allergic reaction during anasthesia, if present, is very moderate, and manifested only by a short period of hypotension. A bacteriaemia is more likely to be related to high pressure injection in operative cholangiography. Different studies report on a more accurate preoperative intravenous cholangiogram compared with the operative one (150), although in general operative cholangiography is regarded superior $(252,253)$.

Operative cholangiography has demonstrated that its use can decrease the incidence of retained stones (RS) $(109,173,34)$. It is safe, and there is no fatality reported with this procedure.

In the jaundiced patient PTC can be performed. A series of 134 patients was reported by Wojtowicz, of which 44 patients obstruction was caused by biliary stones. This study is bias to the liberal use of PTC 14191, because progressive jaundice without cholangitis is seldom caused by CDS. Hence PTC is primarily performed in patients with total obstruction by biliary or pancreatic carcinoma with simultaneously performed internal splintingusing endoprotheses.

PTC can course severe complications (bilio-venous reflux, fatal outcome of pulmonary bile emboli (204), free peritoneal bile leakage,subphrenic or subhepatic abcesses). Schmidt (322) reported a $11.8 \%$ failure rate (which is relatively high in the icteric patient). His complication rate was $6.5 \%$ although in experienced hands it is a safe and effective procedure (412).

ERC is also recommanded to be applied in the same group of patients. However, it is no substitute for operative cholangiography because false-negative retrograde cholangiograms are known [40).

The results of billary surgery in stone disease can be directly related to the quality of operative cholangiography $(46,297)$.

Skillings (347) reported a diagnostic accuracy of $67 \%$. In a retrospective study Sugrue $(365)$, found in $70 \%$ satisfactory informations.

The double standard of results of a radiographic examination of the same organ in the same hospital in and outside the OR is most astonishing:

the quality of postoperative T-tube cholangiograms are particularly superior for the same procedure, but performed in the operation room, with conventional techniques using portable $X$-ray machines.

With these apparates, a blind exposure is employed. One out of 3 films is unsharp because of the long exposure time. It is not uncommon that the procedure has to be repeated resulting in a higher $X$-ray dosage to personnel and the impatient surgeon waiting in a sterile attire. Operative fluorocholangiography with multiple film techniques (6-12 films/patient) is the method of choice as advocated by Berci, Hamlin and Rives 146,2971 . The biliary radicles can be clearly visualized. Sphincter function can be scrutinized on a number of films which in turn helps to differentiate between spasm or stone.

A number of cannulation techniques are recommended to facilitate this part of the 
procedure $145,111,158,162)$.

The advantages of the initial cystic duct cholangiogram for in case of a secondary exploration: the choledocho-cholangiograml are as follows:

1. localisation of stones with special interest to the intra-hepatic ones;

2. anomalies can be discovered in time;

3. observation of concommitant pathology;

4. ductal instrumentation is reduced;

5. iatrogenic injuries can be avoided, especially of the papilla.

Routine operative cholangiography, executed properly, will undoubtedly decrease the total number of negative CDE.

The combined use of initial and completion cholangiograms will further reduce the number of re-explorations. Positive completion cholangiograms without concommitant operative choledochoscopy were found in $4.5-38.7 \%(252,253)$, with an average of $15 \%(173)$.

Table 9.1

Pitfalls in cholangiographic interpretation.

false negative

false positive

-too much contrast material

- the entire biliary system is not visualized

- pour film quality

- pancreatic duct stones

- retained stone(s) in cysic duct stump

- air bubbles

- spasm of sphincter (pseudo-calculus sign)

- poor film quality

It is important to remember that in case of operative cholangiography the initial series of films are the most important ones regarding crucial information lanatomypathologyl to be obtained. In case of a completion study many artifacts are introduced, like air bubbles, floating debries and other artifacts (like blood clots) which makes the life of the radiologist and surgeon alike more difficult. If an initial cholangiogram is available, both sets of films, the initial and completion, can be compared. In addition operative biliary endoscopy is the tool which can help us to clarify the situation in doubtful cases of a completion cholangiogram. This determines the relative value of the completion $T$-tube cholangiogram when operative endoscopy has been performed previously.

\subsection{Antibiotics}

Cholecystectomy and CDE should be considered a septic operation. With older age the total number of patients with positive bile cultures increases from $50 \%$ at 50 to $100 \%$ at 80 years of age irrespectively of concomitant biliary stones $(16,17,75)$.

Partial rather than total obstruction can also be a predilection to infection.

Malignant obstruction is almost never complicated by severe cholangitis [77] 
compared to biliary stones and benign iatrogenic strictures. However, one should be aware about the discrepancies between positive bile cultures and the occurence of (sub-) clinical infection (cholangitis).

Most micromorganism in the billary system are aerobes and only a small number are anaerobes $(108,117,222)$. The composition of biliary bacterial infection resembles the spectrum of micro-organisms found in the duodenum. However, in only $30 \%$ duodenal bacteria can be recovered, but it never harbours anaerobes (256). Biliary bacteria are most likely to be of enteric origin. Consequently anaerobes are supposed to migrate via the portal system.

The correlation of postoperative septicaemia and positive bile culture is well established 131$)$. The majority of infections are established to be of endogenous origin (421). In patients most likely to have positive bile cultures, antibiotic prophylaxis is indicated. These patients are those over 70 years of age regardless of pathology, those with acute cholecystitis and patients with CDS, with or without jaundice. The risks of toxicity or side effects should be minimized in a prophylactic regimen. In-vitro sensitivity of the antibiotic must be relevant to the bacterial hazard. Tissue perfusion should be optimal during operation therefore appropriate timing of antibiotic administration is important.

Although highly effective, aminoglycosides are not prefered in prophylaxis because of their risk of resistence and nephrotoxicity.

They should be preserved for septicaemia.

Karran (269) reported on cephalosporine prophylaxis in biliary surgery. He used one single dose of cephalosporine 2 grams i.m. given one hour before surgery. He reported a considerable reduction in septic complications with no serious sideeffects or any evidence on the development of resistent organisms. Biliary excretion of cephalosporine is poor with consequent minimal bacteriostatic action in bile. Co-trimoxazole was tested in a randomised trial as prophylaxis by Morran (251). One single dose was given preoperatively. A significant reduction in wound infection could be established and less pulmonary complications were seen simultaneously.

Cox 1961 could not show any advantage or disadvantage in the use of prophylactic antibiotics in biliary surgery but the retrospective design of this study limits his conclusions.

High serum concentration of the antibiotic/s) seems more effective in preventing postoperative septic complications than high biliary concentration. In patients with obstructive jaundice antibiotic levels in bile are negligible 1198). Sterilization of bile in the presence of biliary stones, although using the appropriate antibiotic, remains an illusion.

The aim of prophylaxis in biliary surgery is early treatment of bacteraemia which is easily induced by increased intraductal pressure from vigorous intraoperative rinsing, manipulation and instrumentation.

Spillage of contaminated bile should be dealt with by appropriate asepsis and meticulous surgical techniques. In case of intraperitoneal contamination with infected bile, the preoperative administered antibiotic should be continued at least 5 days postoperatively changing from prevention to treatment. 
Vigorous and blind instrumentation can result in severe sequelae like perforation, haemorrhage and late stricture. Vascular complications like portal vein laceration and arterial damage are mostly feared when congenital vascular anomalies in the hepato-duodenal ligament coexist (374).

The methods employed to obtain the objective of removing all possible stones are numerous. A number of devices have been developed, like catheters, bougies. probes, intraductal illumination, cholelithophone, etc.. It is however not the design but the technical skill in using those instruments which determines primarily the traumatic risk and success of the individual instruments employed.

Careful and meticulous surgical dissection is strongly recommended $[82,138]$. The sequential order for stone extraction and peroperative diagnostic aids should be standardized ichapter 10.8).

The instruments used in stone extraction are shown in Figure 9.1. Every procedure should be performed in an atraumatic way. Blind instrumentation should be avoided to minimize iatrogenic damage. If the stone is located, the next instrument of choice is the Fogarty balloon catheter $[130$.. The Randall stone forceps should preferably be used when larger stones are present. Dilatation of the sphincter with Bakes or other bougies is not advisable. This superfluous procedure only increases the risk for laceration of the papil which can result in sphincter fibrosis 11661.

The usual approach for CDE is a supraduodenal choledochotomy halfway between the biliary bifurcation and the sphincter. All stones are usually within the reach of a conventional stone extraction forceps and endoscopic inspection with the rigid cholangioscope. The distal approach, via a papillotomy is used in endoscopic surgery. Every manipulation destroying the anatomy of the sphincteric region can provoke postoperative pancreatitis.

Audrey (15) recommends for stones in the distal duct a supraduodenal choledochotomy and a transduodenal papillotomy. Although the combined technique was used in 42 selected patients only one patient developed acute pancreatitis. No mortality, duodenal fistulae, nor subphrenic abscess or papillary stricture was encountered (391). However, an expeditiously performed transduodenal sphincteroplasty is less likely to induce a postoperative pancreatitis than following a protracted attempt to clear all the distal biliary stones via the supraduodenal route.

The severe complication pancreatitis is less likely to be primarily related to sphincterotomy but rather to the preceding vigorous blind distal instrumentation with a high chance on fausse route resulting in choledocho-duodenal fistulae with septic complications.

\subsection{Complications and failures}

As stated before the majority of operative complications can be prevented by gentle manipulation and appropriate operation tactic. The Fogarty balloon catheter is a 
most useful tool if handled with care. The Fogarty biliary balloon catheter introduced into the hepatic part too far without visual control can perforate peripheral branches, resulting in haemobilia or a bilio- venous shunt. Introducing the same catheter through the sphincter, inflating the balloon and withdrawing it without deflation the sphincter can be severely damaged.

By extraction of large stones using the Randall forceps the mucosa can be caught and perforation can occur.

Extraction of proximal large eroding stones, causing a frail CBD wall can easily be accompanied by perforation and serious portal vein injury (374).

Another serious vascular complication is ligation of the hepatic artery. This is most dangerous in an edematous thickened hepatoduodenal ligament during dissection of an inflammed gallbladder. Partial or total clamping of the CBD in case of inadequate exposure may cause postoperative stricture.

Manipulation especially in the sphincteric region should be performed carefully. Blind instrumentation in this area can result in a choledocho-duodenal fistula which has been more frequently recognized lately by ERCP (183). Forceful dilatation of the sphincter can produce serious iatrogenic injuries (166). Pancreatitis was more frequently reported in those patients where sphincter dilatation was repeatedly employed. Dilatation should thus be eliminated in every common duct exploration.

\subsection{Primary closure versus T-tube drainage}

Choledocholithotomy was already performed in 1894 by Courvoisier. Since then intraductal drainage has been common practise and primary closure is not widely accepted, although serious disadvantages should be considered using a Kehr (TItube. Some authors (88) do encourage primary closure of the CBD.

Reviewing advantages and disadvantages about external biliary drainage, this ancient "biliary amulet should finally be freed from modern biliary surgery" as Kirschner already stated in 1933 (II) (89). However, the controversy still remained: "To drain or not to drain". The main reasons for draining the CBD following choledocholithotomy are:

Advantages:

1. to relieve the elevated intraluminal pressure decompressing a distal duct where papil oedema post-manipulation can occur;

2. to drain infected bile;

3. to perform radiographic studies:

4. to remove retained stones.

Disadvantages:

1. longer hospital stay;

2. interference of sphincter function due to improper position;

3. possibility of (retrograde) infection (344):

4. foreign body reaction of T-tube;

5. hyponatriaemia and hyperchloraemia can occur;

6. fistulae or bile leakage (112); 
7. stricture formation (?) [257):

8. continuous interruption of entero-hepatic circulations with implications on Bile Acid (BA) synthesis (Chapter 2.3)

The need for obligatory postoperative external biliary drainage is primarily related to instrumentation and infection. In a clean case without signs of cholangitis, sphincter function assessed by operative fluorocholangiography, primary closure can be reliably performed. However, it is still a sound safety valve to have an indwelling catheter inserted for approximately one week in the postoperative period. Adequate draining of the gallbladder bed is recommended in all cases if no T-tube is inserted, which can be removed within 24 hours. 


\section{Chapter 10}

\section{Cholangioscopy}

\subsection{History}

The first experiments with cholangioscopy date back more than fifty years. In 1923 Bakes published his first experience with cholangioscopic examination of the CBD (22). This surgeon, practising in Vienna, had his prime interest in biliary surgery. His name has been permanently associated with biliary surgery through the developement of his bougies.

it is good to remember that these endoscopic experiences were performed even before the introduction of intraoperative cholangiography (248).

The proposed endoscopic procedure was an indirect visualization of the interior of the biliary tract, comparable to indirect laryngoscopy. This splendid idea concerning visual inspection of the lumen of the duct did not gain wide acceptance at that time because of lack of technology. The first cholangioscope consisting of a right angled telescope with an electric globe was published by Mclver in 1940 (243). Since 1953 the real promoting of cholangioscopy has been done by Wildegans in Berlin 1414 . He used a $60^{\circ}$ angled telescope, which he claimed to be superior to intraoperative cholangiography 1413 ). His instrument was not widely accepted because of technical shortcomings, primarily concerning the standard optical system employed. This instrument was significantly improved by Berci in 1960 when he introduced the Hopkins rad lens system to endoscopy 144,3381 . Because of this technical innovation the diameter of the scope could be decreased. The improved technical features in combination with the superior optical system made the rigid instrument a highly efficient apparatus which enables the biliary surgeon to carry out detailed visual examination of the major bile ducts (415).

In 1965 a flexible cholangioscope was introduced by Shore $[336,337)$. Equivalent clinical results were obtained $(127,154,197,280,360)$. Comparative studies have been performed with both rigid and flexible scopes (185): the rigid scope was preferred for routine use because of simple instrumentation, fixed focus and superior optics, facilitating intraluminal orientation and easy manipulation, with a minimal change on fausse route.

The rigid scope can withstand repeated sterilization unlike the flexible scope through its delicated nature limiting the number of applications. The flexible scope clearly more expensive is obvious more vulnerable.

The rigid cholangloscope can be used also in urology for inspection of the pyelum. incidental application in vascular surgery to visualize the anastomosis has been performed $(384,385,395)$ but peroperative angiography seems superior to test the patency of the anastomosis.

\subsection{Cholangioscope and accessoires}

The vertical limb measures $38 \mathrm{~cm}$. The horizontal limb is 4 or $6 \mathrm{~cm}$ (fig. 10.1). In 
biliary surgery the $4 \mathrm{~cm}$ scope is particularly useful for inspection of the distal duct The maximum diameter of the horizontal limb is $5 \times 3 \mathrm{~mm}$ and comprises the optics, irrigation-system and light channel (Fig. 10.2)

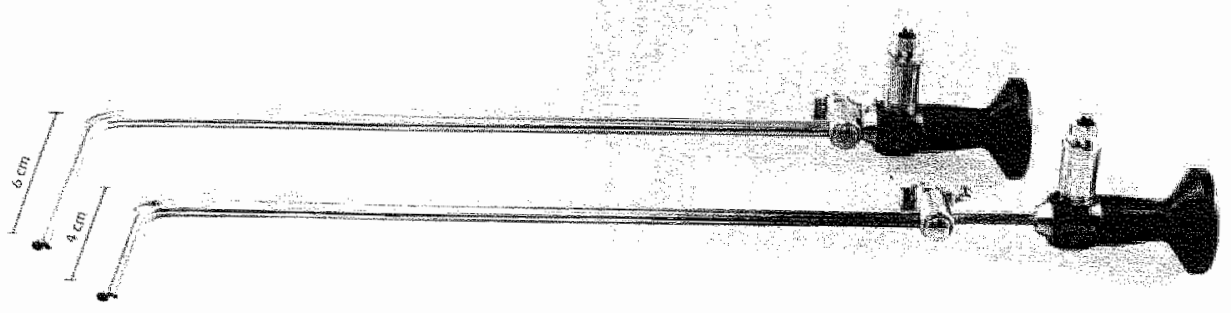

fig. 10.1: Storz Cholangioscopes, 40 and $60 \mathrm{~mm}$.

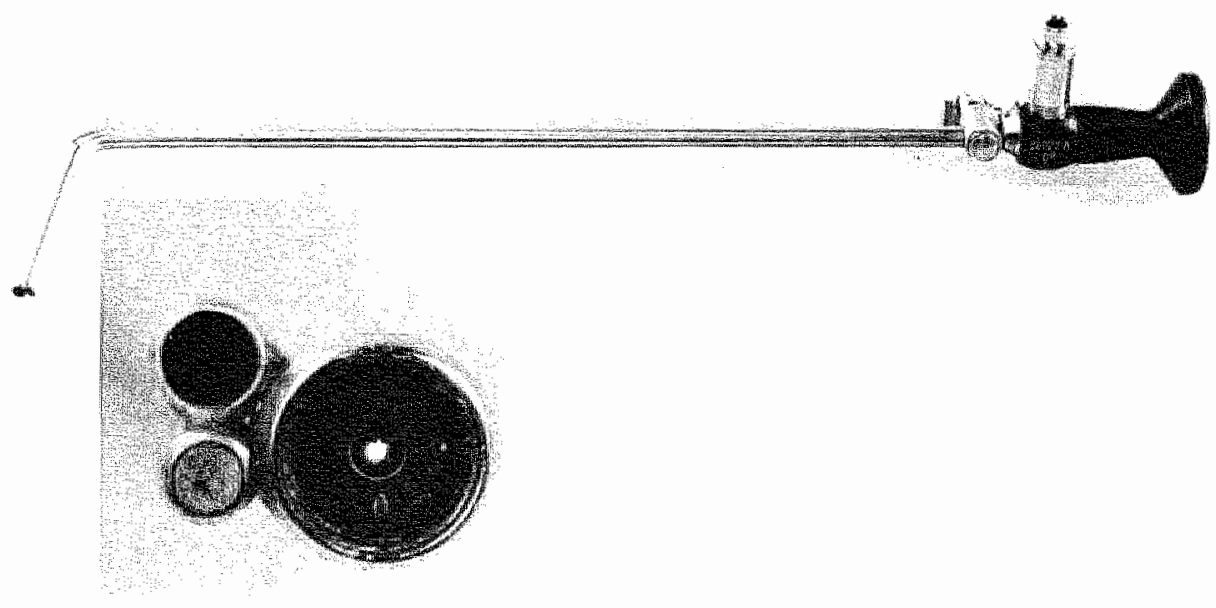

fig. 10.2: Front detail of cholangioscope

Several accessories are available: two rigid stone forceps are shown in Fig. 10.3. Flexible accessories like the Dormia basket can be introduced through a separate guide channel which can be attached to the scope (Fig. 10.4). Flexible stone forceps as well as Fogarty arterial bafloon catheters size Fr. 4 and even diathermy cabies can be used |Fig. 10.5), taking the same instrument guide channel. 

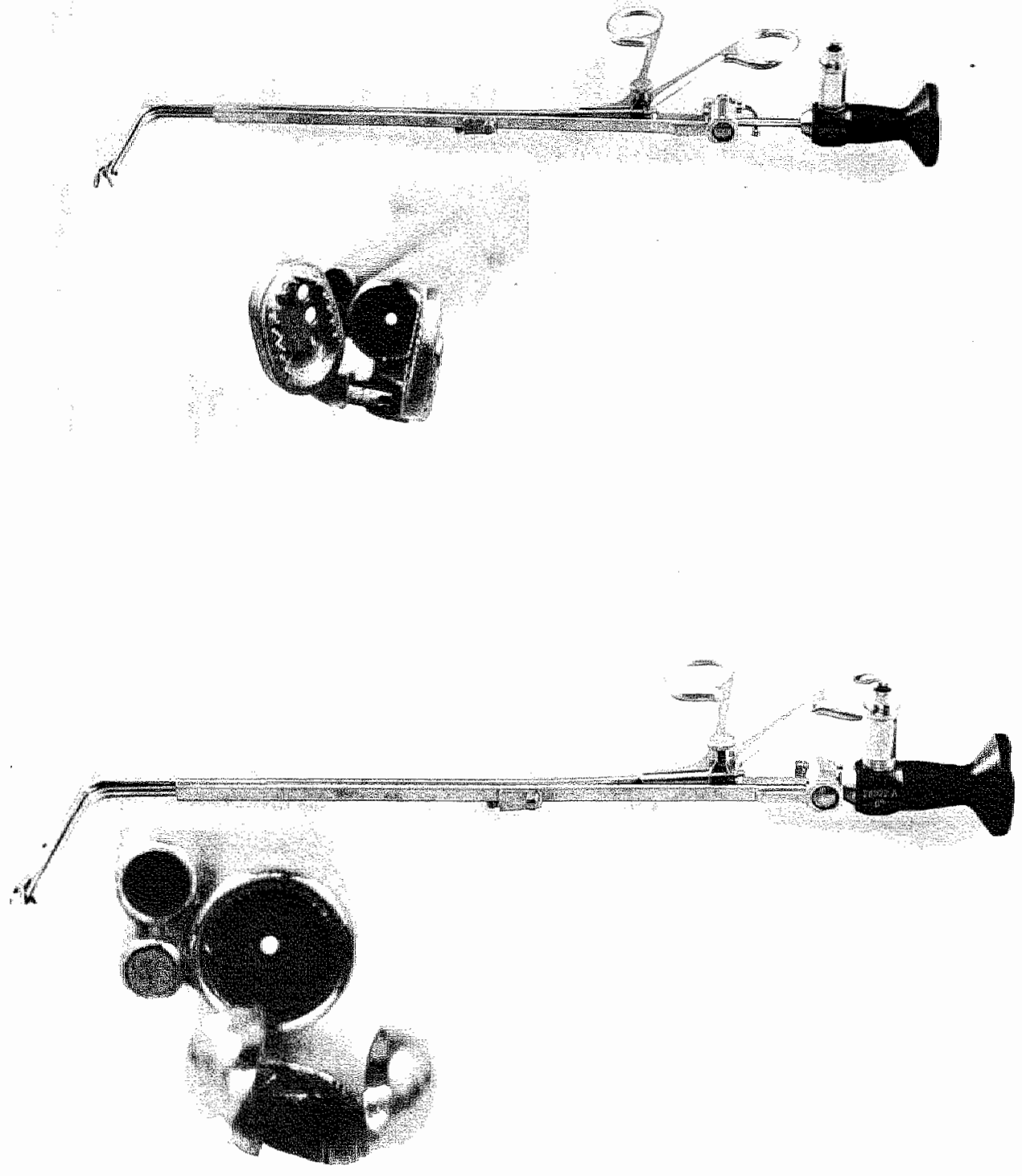

fig. 10.3: Rigid Stone forceps 


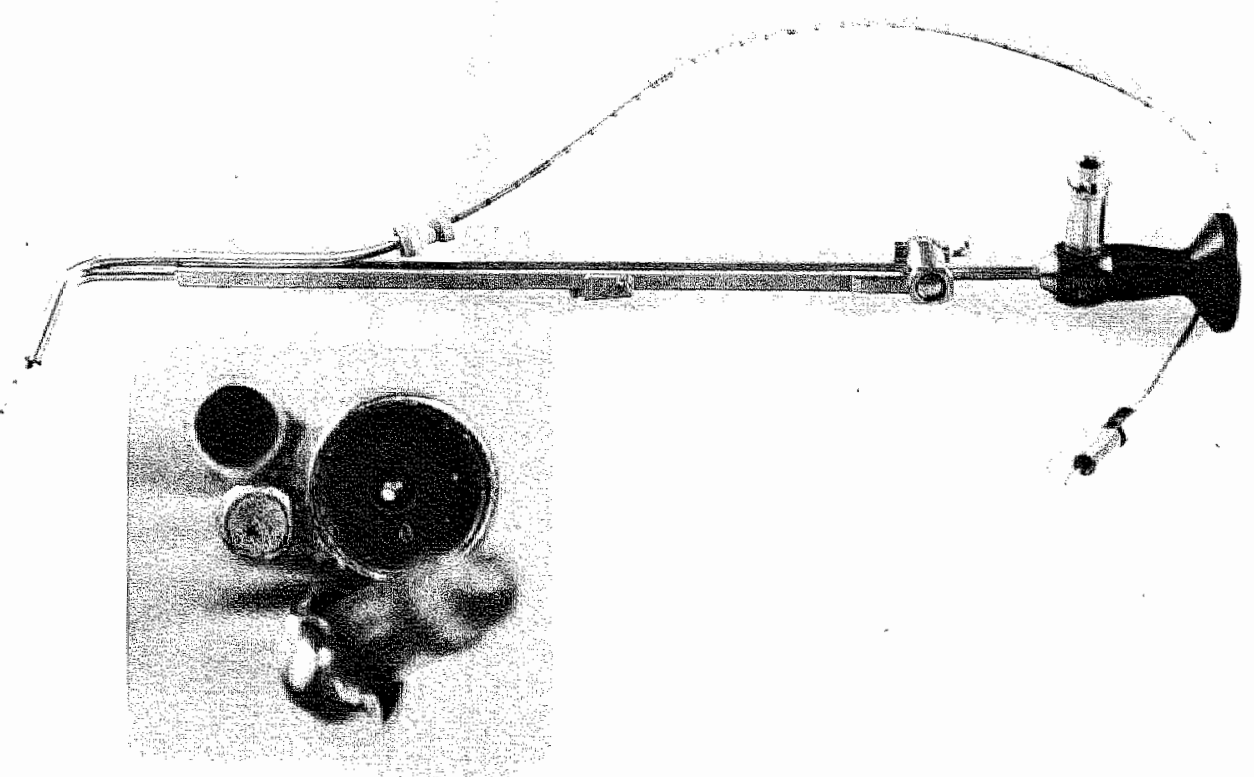

fig. 10.4: Guide channei with Dormia basket

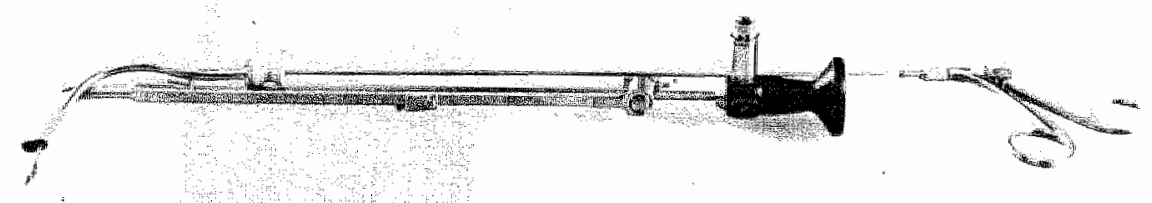

fig. 10.5: Flexible stone forceps

To increase the light transmission a specially designed fiber cable can be attached to the scope to facilitate still photography (Fig. 10.6).

A disc attached to the eyepiece is indispensable to maintain sterility during the entire procedure (Fig. 10.7). 


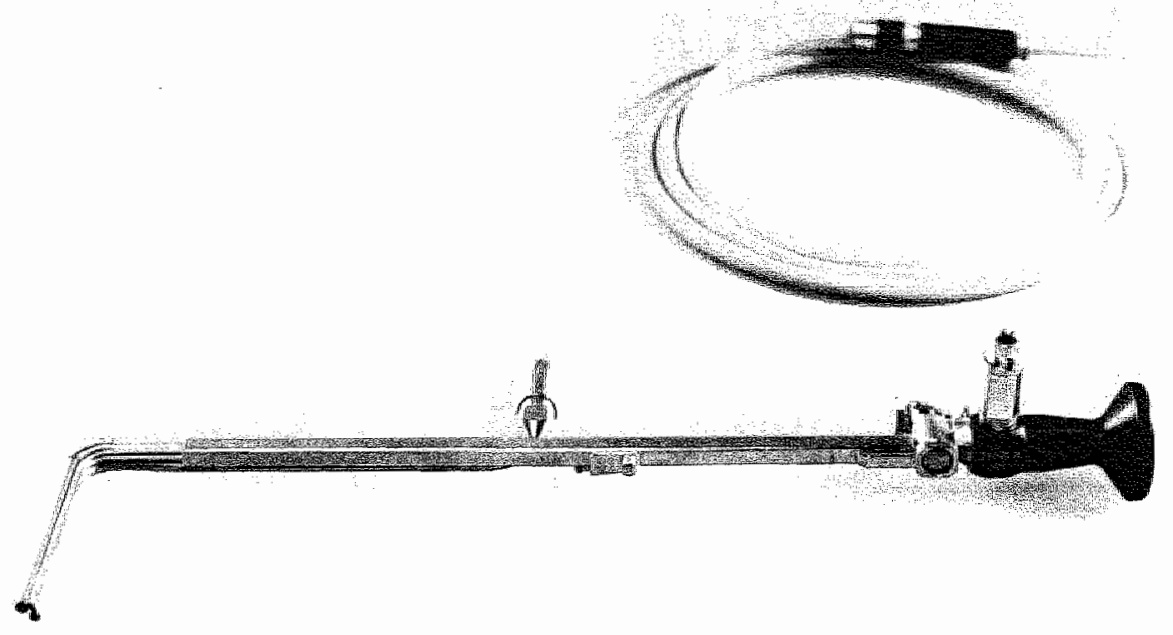

fig. 10.6: Fiber cable for still photography

酒.

.

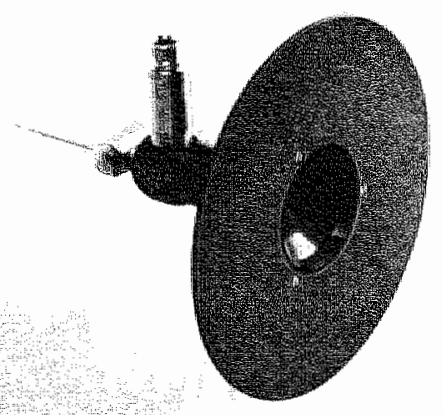

fig. 10.7: Eye disk

The light source consists of a halogen $150 \mathrm{~W}$ projector globe. The illumination is transmitted via a flexible fiberoptic cable to the right angled cholangioscope (Fig. 10.8).

For reasons of instruction it is important the assistent to follow the operator"s 
movements in the interior of the biliary system. (fig. 10.9)

For this reason a teaching attachment was developed which permits simultaneous observation for both operator and assistent. The image on both ends are equal, a distinct advantage over the flexible teaching attachments. Moreover, it can be gas sterilized.

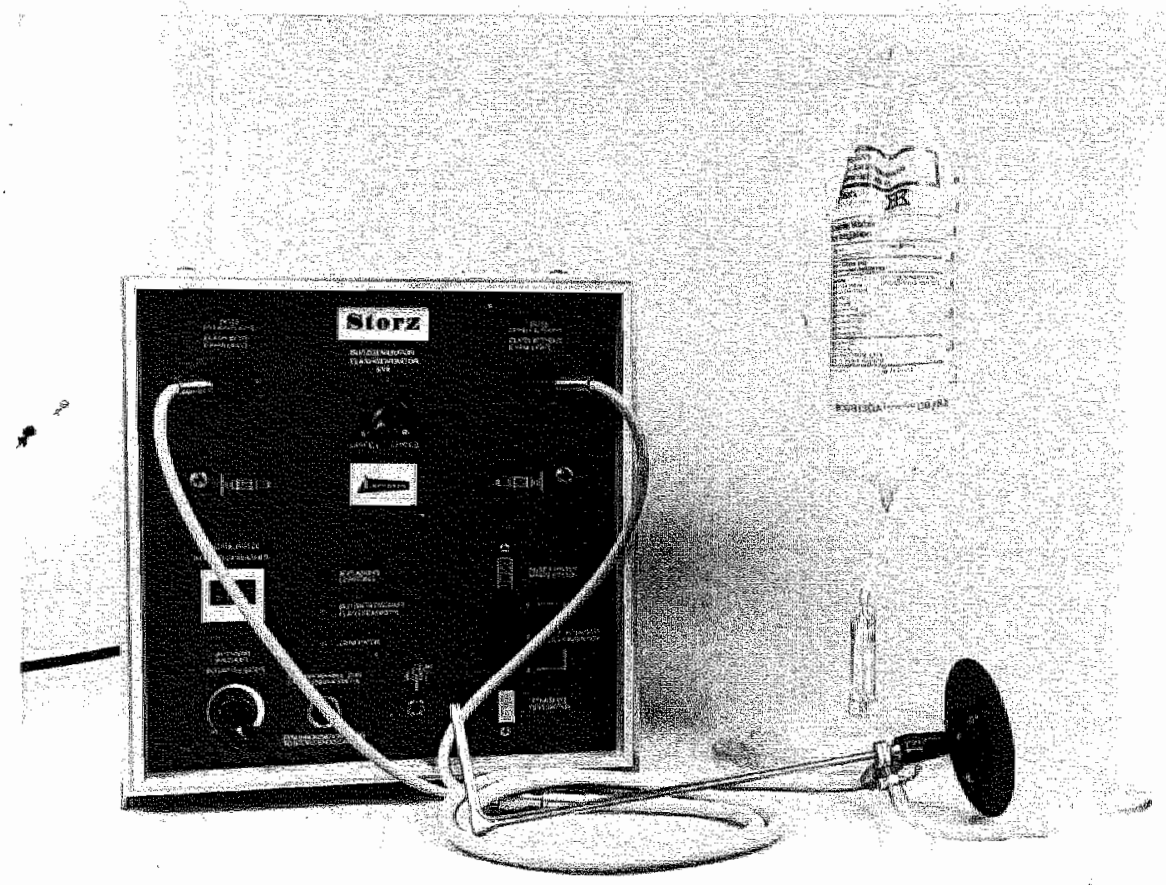

fig. 10.8: Complete cholangioscope set

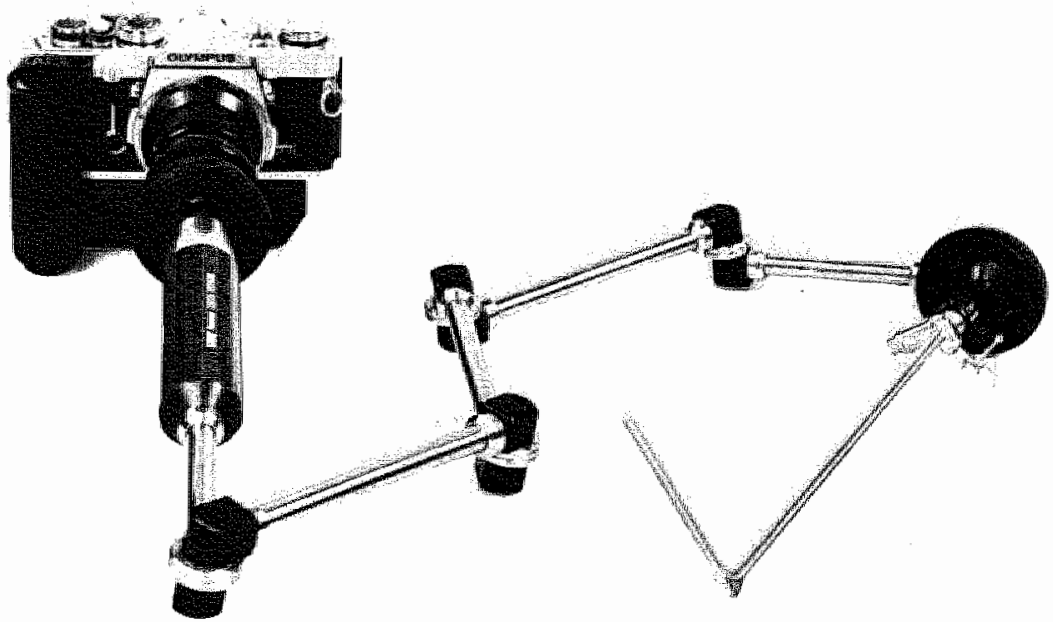

fig. 10.9. Articulated witmoser teaching aid 


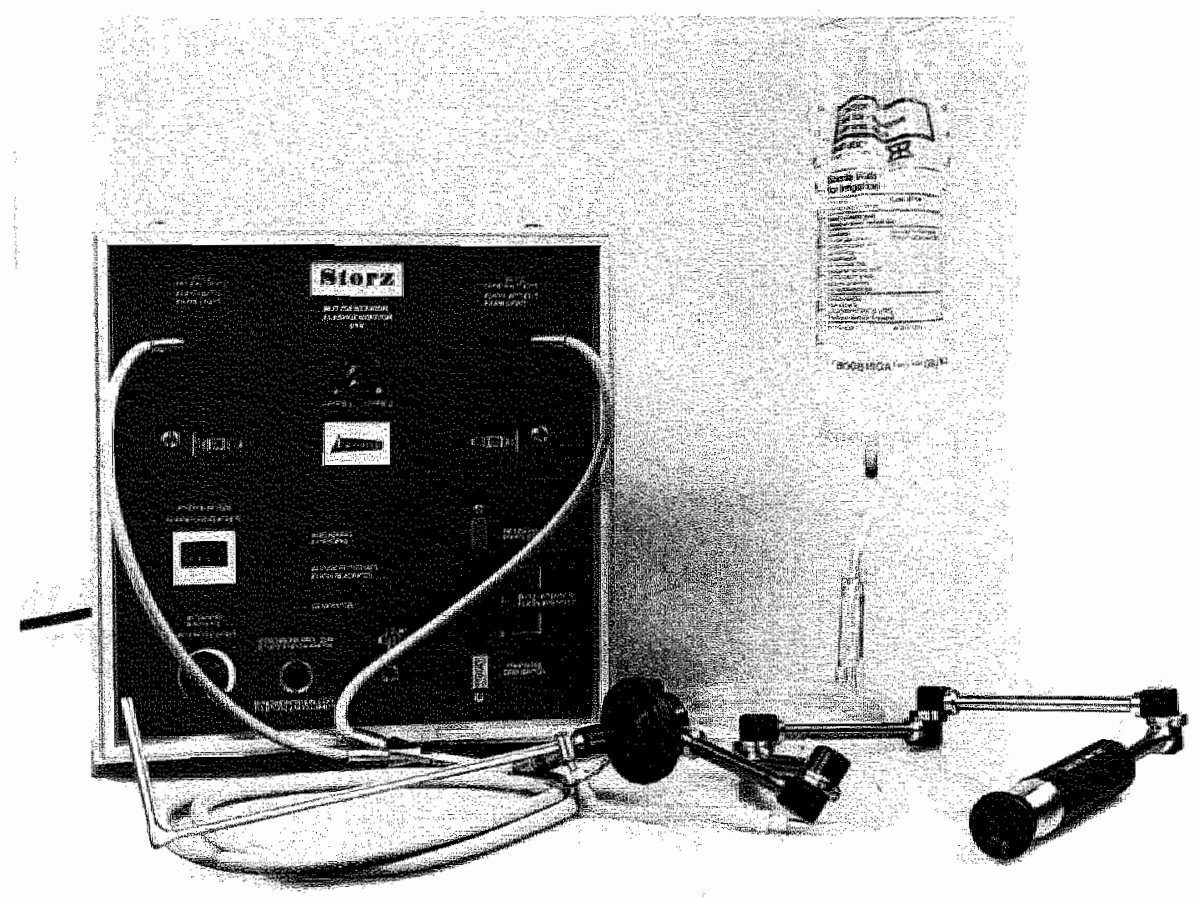

fig. 10.9: Articulated Wittmoser teaching aid

An articulated arm with optical joints has been produced to compensate movements and still be able to preserve the image quality of the Hopkins rod lens system. A double beam splitter divides the image $50-50 \%$ in case of simultaneous teaching and by inserting the other beamsplitter $90 \%$ is transmitted to the camera which at this stage substitutes the second abserver. In this way photography or television transmission can be realized without interference of the routine. Irrigation is a prerequisite to obtain a clear view of the biliary system. Sterile saline solution is used. Hydrostatic pressure is provided by elevating the battle $1.25 \mathrm{~m}$ above the patient. High pressure irrigation with a bulb pressure gauge is not necessary. Intraluminal pressure may never exceed $30 \mathrm{~cm}$, which will probably not be reached because of two functional safety valves: the choledochotomy incision and $a$ functioning sphincter. Nevertheless, if this pressure is reached, cholangiovenous reflux might initiate a bacteriaemia $(270)$.

This is of particular interest in retrograde endoscopic techniques (ERCP)and postoperative endoscopic stone extraction with the flexible cholangioscope via the T-tube tract (see Chapter 11.3 ). During those techniques irrigation is performed in a closed billiary system. Transient or even septic fever can occur in the postmanipulation phase.

The amount of irrigation fluid during cholangioscopy should be measured and the hemodynamic status corrected according to need in the early postoperative period. Especially in elderly patients fluid overload can result in heart failure. 


\subsection{Sterilization}

Gas-sterilization (ethylenedioxide) is considered the optimal method for this optical system amongst the sterilization techniques in use.

Steam autoclaving can be harmful for the delicate optical system.

Gas-sterilization needs 24 hours which is a disadvantage because the cholangioscope is not immediately available. In these few instances soaking 20 minutes in glutarformaldehyde solution can be employed.

Careful rinsing is required. At least three sets of cholangioscopes are helpful to guarantee a 24-hour availability in case of biliary emergencies or to have a back-up set in case of repairs.

\subsection{Indication}

A common bile duct exploration is incomplete without cholangioscopy. The only exception to abandon cholangioscopy is a too small bile duct system where the introduction of the scope can be risky. This is very uncommon when stones are present in the billiary sytem.

Cholangitis is not a contraindication. The duct in these cases has to be cleaned by rinsing out the debris to allow proper visualization and all coexistent stones have to be retrieved.

If indications are present to perform a billio-enteric bypass, cholangioscopy should still be attemped to obtain all possible information about the status (stones, mud, cholangitis) of the hepatic as well as of the distal duct. Stones should be removed if necessary under visual control.

If this type of duct cannot be cleaned out completely (this may be one of the reasons to bypass) it is important to obtain as much information as possible about the distal duct to relief or prevent a future sump syndrome. That is another reason to perform cholangioscopy even in these patients. Bypass surgery is no alternative for operative cholangioscopyl

In cases of chollangiocarcinomas, cholangioscopy can be of assistence to establish criteria for primary resection (54). Hilar cholangio carcinoma of Klatskin(389), not extended to the first radicles of the left and right hepatic ducts, can be proven by endoscopic biopsy.

In other words, cholangioscopy is important in staging, establishing criteria for localized or disseminated lesions (382), deciding on palliative treatment or curative resection.

\subsection{Technique}

After choledochotomy the Kocher manoeuvre to mobilize the duodenum is not always necessary but facilitates the distal endoscopic exploration considerably. By stretching the duodenum the distal choledochus is straightened, thereby facilitating the inspection of the distal common blle duct. 
The incision is carefully placed and not exceeding $1 \mathrm{~cm}$. if a too proximal choledochotomy is made the introduction of the right angled apparatus is hindered by the costal margin.

A too distal incision on the other hand will make the proximal inspection difficult. The optimal incision should be placed in the middle of the supraduodenal part of the common bile duct.

The cystic duct cannot be used as a reference point for the incision because of the wide anatomical range of the insertion into the CBD (Chapter 8.2).

The incision should not be extended to introduce the scope: a long incision can make intraductally vision troublesome, by using too much irrigation fluid leaking through the choledochotomy.

The $1 \mathrm{~cm}$ incision is sufficient. Choledochotomy is made in between two stay sutures which are crossed after introduction of the scope to avoid spillage of the irrigation saline providing adequate distension of the ducts. A semi-closed system is created with normal intraluminal pressure.

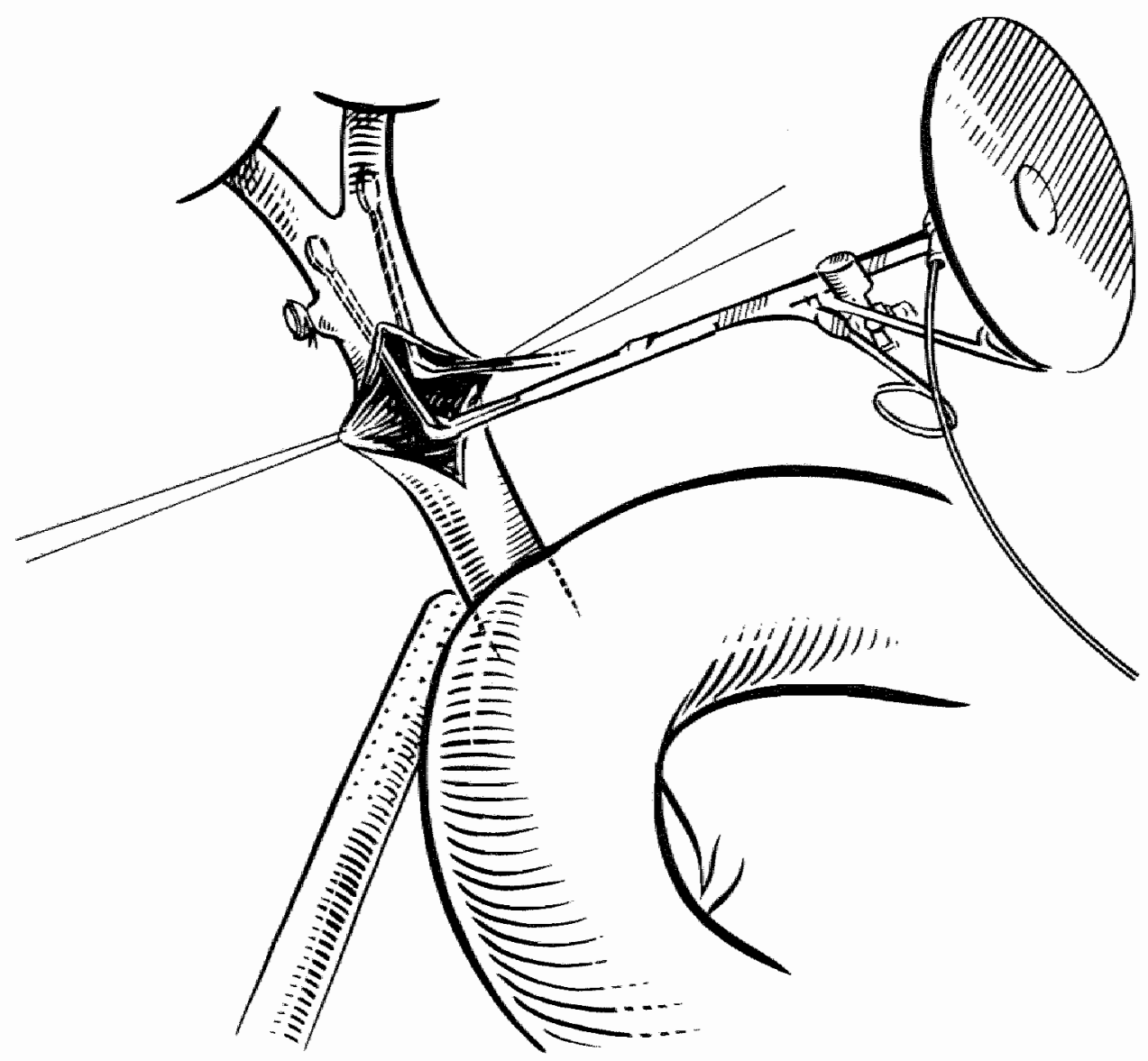

fig. 10.10: Introduction of the scope 


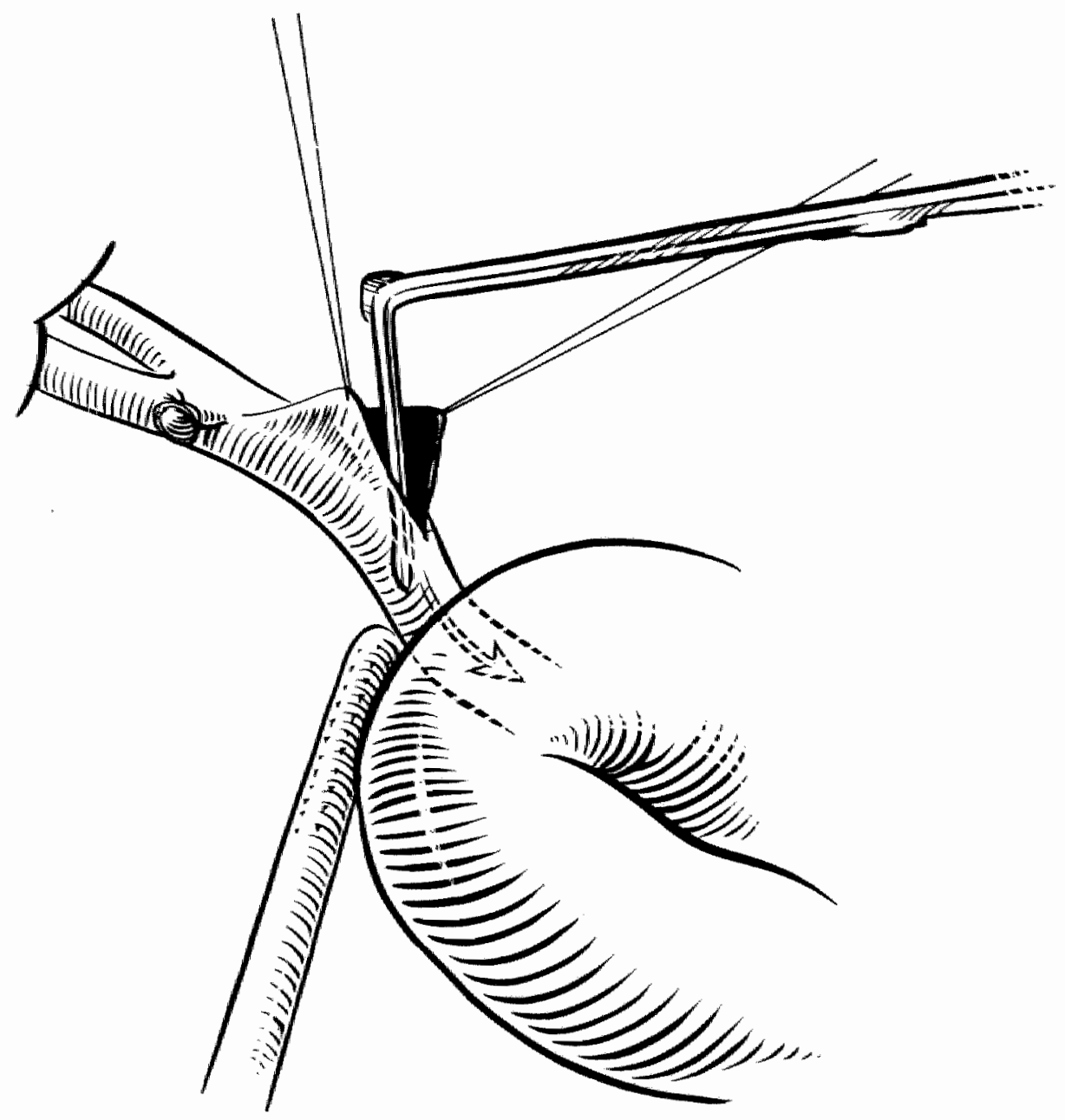

fig. 10.10: Introduction of the scope

Septic complications may thus be prevented [Chapter 10.9).

To diminish the angle between scope and common duct during introduction, the common bile duct is lifted by the stay sutures. Damage to the posterior wall is thus reduced to a minimum. [fig. 10.10).

The scope is introduced under direct vision to avoid missing calculi between the ductal wall and scope. The irrigation stopcock is opened when the scope is inserted. When the lumen can be indentified the scope is advanced. One hand is tilting the vertical limb holding it below the irrigation connection. A safe distance from the eyepiece and operator's head is required. The second hand is on the duodenum keeping it on a continuous stretch. If inspection of the proximal part is completed the scope is reinserted into the distal part. 
The spillage of irrigation fluid beside the scope is continuously aspirated. The infusion bottle should be placed at $2 \mathrm{~m}$. height on a I.V. stand (see Chapter 10.3).

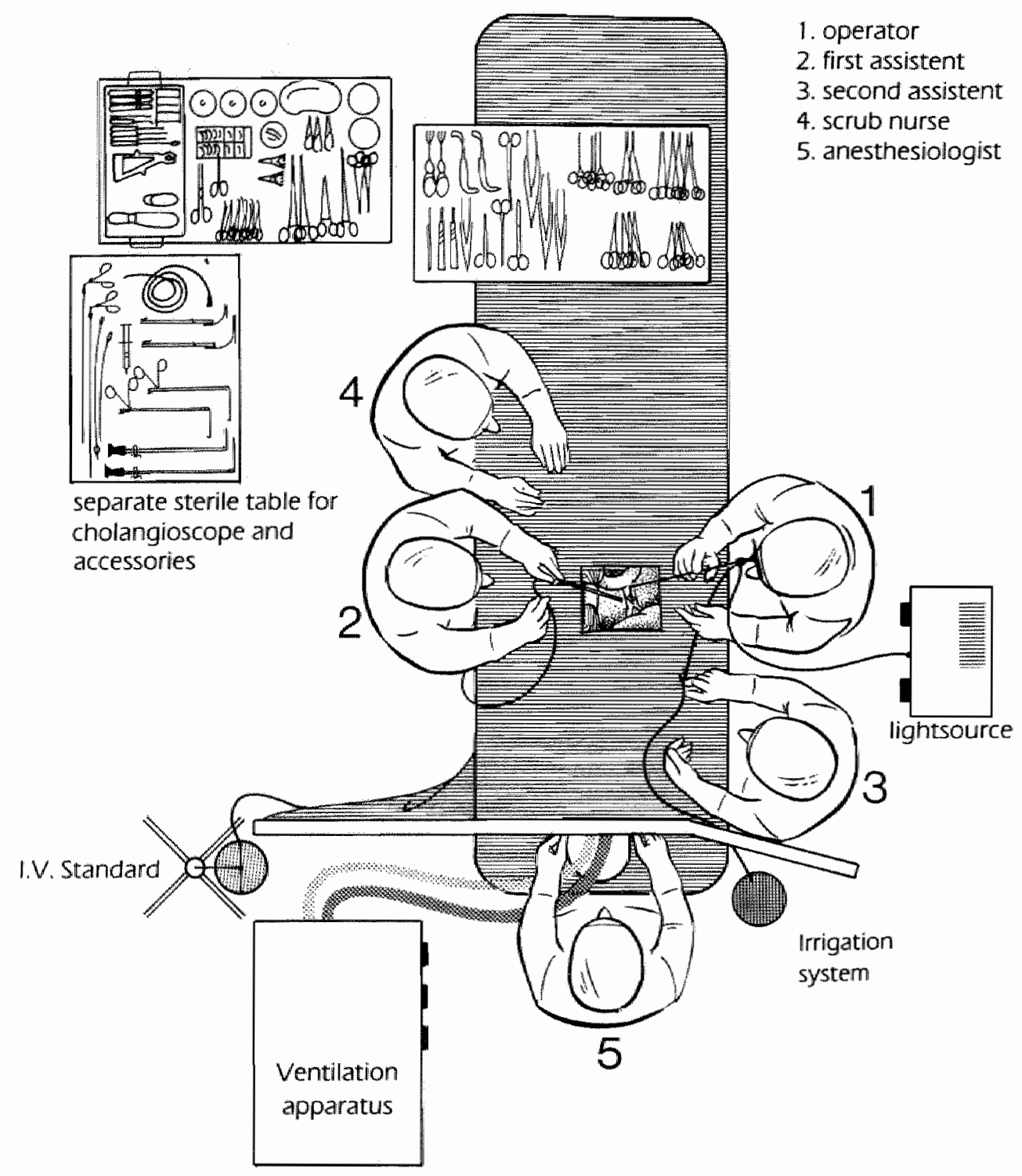

fig. 10.11: Operative biliary endoscopy in operation on the $O R$ 
The assistent retracts the liver and provide suction. When no appreciable vision is obtained because of a wide duct or spatulous sphincter, a Fogarty balloon catheter can be introduced alongside the scope occluding the distal sphincter.

W/hen the length of the horizontal limb of the scope $(60 \mathrm{~mm})$ is still too short for optimal inspection the duodenum can be "gloved" around the scope.

Respiration may severely hinder proper inspection of the sphincter region which can be dealt with by asking the anestesiologist for a few short apnoe phases.

When the scope is incidentally touching the wall a red or yellow disc is seen (Fig. 10.12). Orientation is quickly achieved by withdrawing the scope slowly under visual control. A clear image of the lumen will appear. The scope is advanced towards the sphincter.

Carefull inspection and observation of this important area is carried out. The proximal system is easier to examine because it is embedded in a solid organ (liver) and the lumen does not collapse immediately.

The distal part needs more preparation like Kocherization, stretching the duodenum and distension with irrigation fluid.

If all these steps are followed a splendid view of the interior of the biliary system will be obtained.

\subsection{Findings}

The entire ductal system from the sphincter to the intrahepatic tertiary branches must be inspected (268).

Attention should be paid to the appearance of the mucosa: hyperemia, debris, cholangitis, arteficial lesions caused by instrumental manipulation (Fig. 10.13). Anatomical landmarks make orientation easier: the orifice of the cystic duct, the ampulla of the distal part just proximal to the sphincter (Fig. 10.14), opening and closing functions of the papilla and occasionally the orifice of the duct of Wirsung. 
Proximal ducts: after introduction the scope is advanced under direct vision towards the bifurcation. The intrahepatic ductal system should be investigated systematically $(319,320,402)$ : bifurcation and the left and right hepatic ducts with the ventrocranial and dorso-caudal branches, both with its tertiary channels (Fig. 10.15). The scope is slowly withdrawn from each hepatic duct back to the bifurcation. Then the distal duct inspection is carried out. No forceful dilatation should be performed neither with Bakes bougies, nor with the scope itself (see Chapter 9.5): a visual prolonged view of the ampulia is sufficient to assess the function of the sphincter.

\subsection{Tactic of cholangioscopy}

The order of sequence of the different techniques in CDE includingcholangioscopy is of paramount importance to achieveoptimalresults(Table 10.1).

Table 10.1

Recommanded order of instrumentation in CDE

-1: Initial (cystic duct) cholangiography

-2: $\quad$ Stone extraction

-3: cholangioscopy

-3a: Overlooked stone removal

-4: Completion T-tube cholangiography

For legend see text (Chapter 10.7)

If a preoperative clinical indication to explore the biliary duct can not be supported by a positive operative cholangiogram, the first step in CDE should be cholangioscopic inspection (Table 10.2). Do not start a blind lengthy manipulation phase which can interfere (bleeding) with a later performed cholangioscopy.

Operative cholangiography is the method of choice to localize the stones in number and topography. If the number and the location of stones is not known extended instrumentation is not uncommon with increased risk for injuries. On completion cholangiograms debris, blood clot and created artefacts can mimic a stone. These are the cases where cholangioscopy can solve this dilemma with an added few minutes to the operating time.

After mechanical extractions are completed the scope is intro- duced. If no residual stones are seen the operation can be terminated with a T-tube insertion. The choledochotomy is closed with interrupted catgut sutures.

If overlooked stones are discovered on the completion cholangiogram they can still be retrieved under endoscopic control.

In secondary CDE an initial choledocho-cholangiogram should be per-formed first. This prospective protocol on CDE including cholangioscopy has been employed in a clinical study for evaluation of the use of cholangioscopy (Chapter 13). 


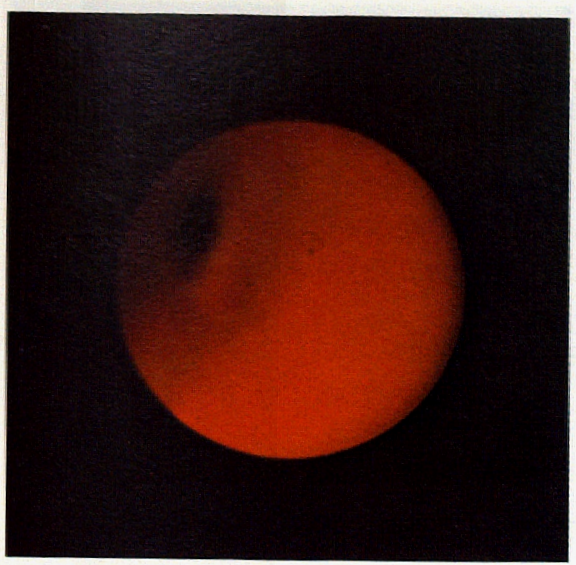

fig. 10.12: Cholangioscope close to ductal wall

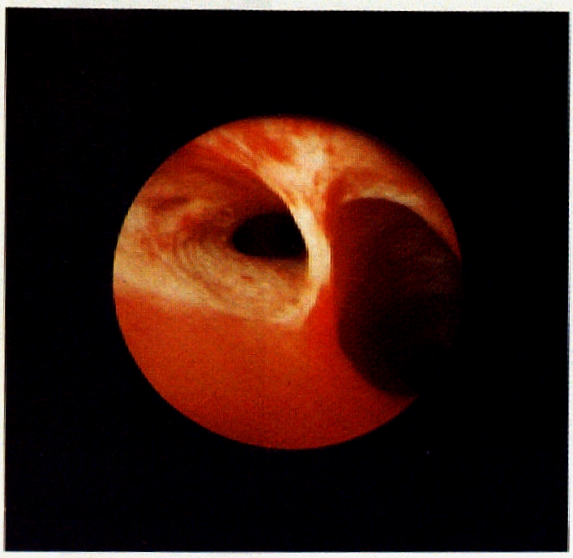

fig. 10.13: Mucosa defects by stones and instrumentation

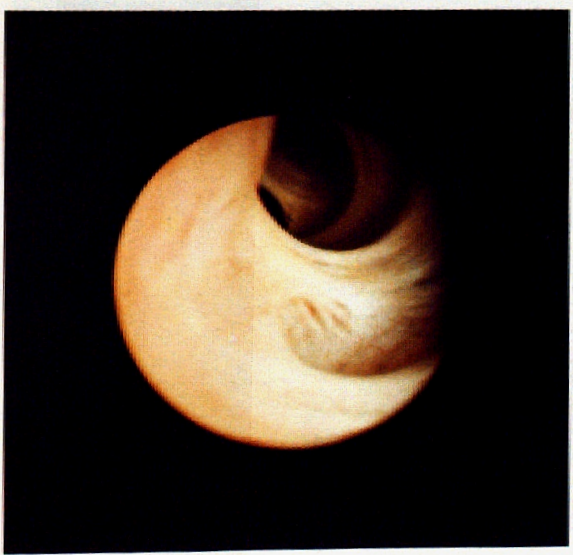

fig. 10.14: Proximal biliary system 


\subsection{Complications}

Complications following operative biliary endoscopy are seldom reportedwhich is in accordance with our limited experience over a time period of 4 years in 162 cases (Chapter 13).

Perforation is feared with every probing instrument if it is in-

appropriately used. The cholangioscope should always be introduced and handled gently under visual control.

The operating time is prolonged with 10-15 minutes, but with more experience this can be reduced. A 10-15 minutes extention of a 1-2 hours procedure does not influence the final outcome markedly.

The obtained information and results far outweight the extention in operation time. As will be outlined in chapter 13, the incidence of septicaemia is not increased because of the use of biliary endoscopy. Administra- tion of antibiotics because of cholangioscopy itself is not indicated. 


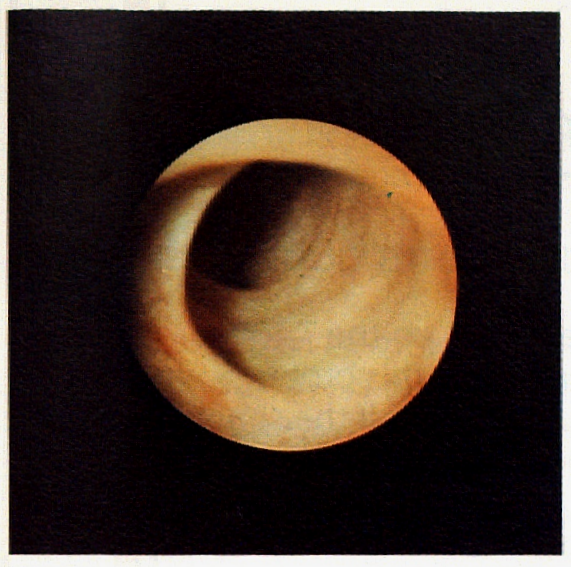

fig. 10.15: Distal CBD; normal mucosa

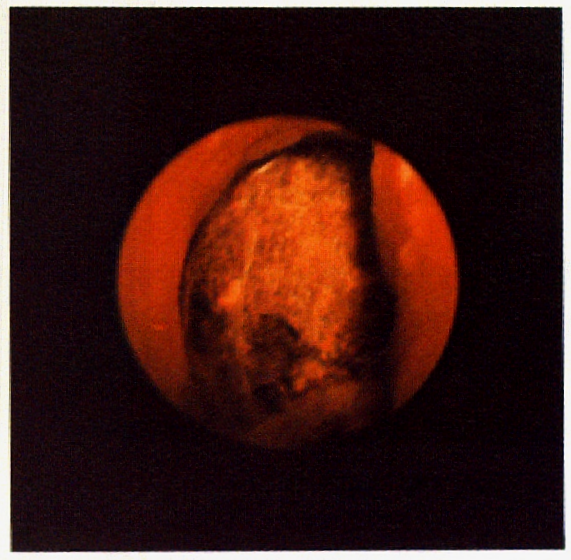

fig. 10.16: Close-up of Large CDS

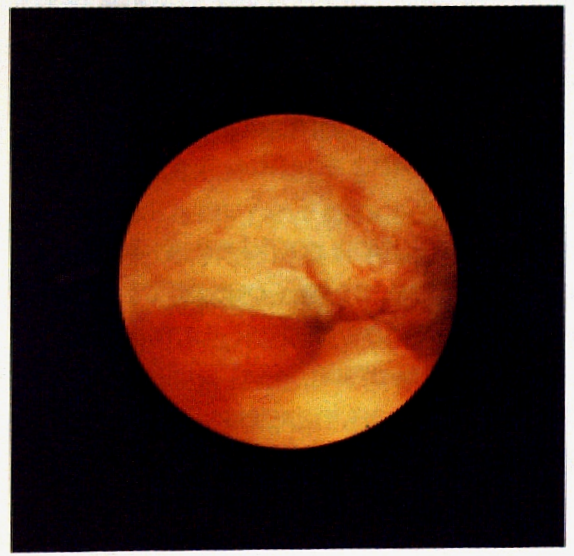

fig. 10.17: Pancreatic tumour compressing the CBD 


\section{CHAPTER 11}

\section{Non-operative treatment of Retained Stones}

\subsection{Decision making}

If a stone is discovered in the postoperative period on the T-tube cholangiogram there are various ways to approach this problem: a. spontaneous passage can occur with smaller stones if the sphincter function is intact. Therefore a waiting period of $4-6$ weeks with indwelling and well preserved T-tube is advisable;

b. if the patient does not tolerate the clamping of the tube it is to be considered as a sign of the necessity of intervention, non-invasive or invasive.

There are several reasons to postpone an immediate reexploration for retrieving (an) overlooked stone(s).

A higher morbidity and mortality is encountered with every second exploration.

Three methods are available to treat patients with RS (Retained Stone(s) and indwelling T-tube:

1. chemical dissolution:

2. stone extraction through the T-tube tract;

3. endoscopic papillotomy.

\subsection{Chemical dissolution}

Chloroform and ether installation have been abandoned because of pain, complications and ineffectiveness.

In 1938 Best started with chemical dissolution of retained gallstones using hydrochloric acid combined with sphincter relaxing agents and food, known as "Best's biliary flush" (51).

Oral administration of Bile Salts, mainly Cholic Acid, has also been used in patients with retained stones in a dosage of 3-4 gr per day (Chapter 9 and 13).

In 7 of 9 patients radiographic stone disappearance could be established within 8 13 weeks (399).

Heparine has also been proposed as a potent stone dissolving agent. In vitro it is totally uneffective to dissolve stones (383).

Not even an enhanced effect of heparine in bile salt dissolution could be shown (85). However, Christiansen (86) reported successful treatment in 3 out of 7 patients. Diarrhea is a side effect is which can be depressed by cholestyramine. The effect of heparine in dissolution of stones is still controversial.

Monooctanoin (Capmul), a glycerol derivate, appears to be an inexpensive and most effective cholesterol solvent amongst all other agents registered.

It has been tested clinically (375) and successful dissolution of stones within 4-21 days has been reported. 
The patient has to be hospitalized for a period of $1-2$ weeks, repeatedlaboratory tests are required to control side effects of liverdamage.

\subsection{Stone extraction through the T-tube tract}

Residual stones can be successfully removed under fluoroscopic control via the $T$. tube tract in $90-95 \%$ of the patients. The morbidity is in the vicinity of $5 \%$. It is a safe and fast technique, which can be performed as an outpatient procedure: Mazariello (239) and Burhenne $(72,73)$ published large series of with excellent results. The main complications are perforations of the tract and bile leakage. This can be dealt by reinsertion of a drainage catheter under fluoroscopic control. The patient should be covered with antibiotics. A mild cholangitis is not uncommon. In $2 / 3$ of the cases a second or third session is required (86).

A flexible fiber cholangioscope has been also applied with great success $[187,261\}$. Radiation hazard and the time required for the completion of the procedure has been considerably reduced.

The surgeon has to know that in doubtful or difficult cases a large caliber T-tube (Fr. 18-20) should be used. The horizontal limb should be short and the upper half removed. The tube should be brought out through a separate stab incision in the flank.

The tube may not exit the incision or midline. The route of the vertical limb should be straight because a curved small sinus tract will make postoperative stone extraction difficult if not impossible.

\subsection{Endoscopic retrograde sphincterotomy (ERS)}

Since the introduction of ERCP diagnostic accuracy of the biliary system has been increased. In experienced hands this technique carries a minimal morbidity (406). In 1973 the first reports on EPT were published (118).

The overall success rate including morbidity and mortality needs further attention. Even in selected patients this examination has significant complications and a relatively high mortality rate $(1,1-4 \%)$. This endoscopic procedure should be preserved for trained endoscopists.

in case of complications surgery should be performed without delay to prevent serious septicaemia.

It is a relatively new procedure which will need years of well documented follow-up studies to compare the final results $(75,295,305)$ with surgery.

Cotton (93) presented a group of 134 cholecystectomized patients, so called, high risk cases. The majority of these patients had withstand cholecystectomy without complications recently. The selection of this type of patient in favor for endoscopic surgery is questionable. In 85 patients of this series the CBD was not explored nor was operative cholangiography performed. Routine use of this technique undoubtedly could have reduced the indication for endoscopic surgery considerably. The overall incidence of RS including the failure rate of EPT was $11,5 \%$. These data compete with "second class" surgery. 
Infectious complications have been reported to decrease using prophylactically antibiotics in ERCP and EPT (184). In 8\% of all upper Gl procedures, bacteriemia has been demonstrated (211).

Administration of antibiotics seems to be justified. The net effect of installation of antibiotics in the biliary system following EPT, providing free bile drainnage, equals one oral dose of antibiotics. Parenteral administration is thus preferred, especially in patients suffering from heart valve disease.

Blumgart (56) demonstrates excellent results in (diagnostic) ERCP with no complications comparable to a $3 \%$ complication rate in other studies. His operative results were even more impressive. He states that precise surgery guided by exact preoperative information is the safest procedure.

ERCP has thus been established a reliable and safe procedure indicated in problematic biliary and pancreatic disorders not otherwise diagnosed by noninvasive techniques.

Whether EPT is an alternative for surgery as a definite curative procedure is still questionable. It is obvious that the very sick and high risk patient can benefit from this technique even as an intermediate step in guiding the high risk patient to an improved physical condition to undergo elective surgery. 


\section{CHAPTER 12}

\section{Operative treatment of Retained Stones}

\subsection{Re-exploration}

A considerable number of Retained Stones (RS) are large calculi (72,305), sometimes almost obstructing the CBD. Over $10 \%$ of all RS were measuring $1 \mathrm{~cm}$ and almost half of the stones retained were greater than $0.5 \mathrm{~cm}$ 147!. Nevertheless, spontaneous stone passage was seen in 24/69 patients by Bartlett.

Two stones were as large as $1 \mathrm{~cm}$ although stone passage was reported not until 3 months postoperatively. Retained stones almost never cause severe cholangitis or biliary obstruction necessitating early re-exploration. Re-exploration is thus strongly recommanded to be postponed until at least 6 weeks postoperatively, and noninvasive methods should be tried in the meantime $(25,247)$.

CDS and consequently RS are most frequently seen in older patients with coexistent underlying diseases: cardio-vascular, pulmonary and centrall nervous system, all adversely influencing the morbidity and mortality. This was illustrated by Bartlett (25) who reported three fatal outcomes in these patients, not related to biliary surgery.

Re-exploration carries a higher morbidity and mortality. Therefore, if re-exploration is unavoidable this should be a definite procedure.

\subsection{Bilio-enteric bypass}

The same drainage procedure performed in biliary and pancreatic carcinomas as a palliative measure is consequently omitted and are beyond the scope of this chapter. There are four anastomatical sites suitable for the procedure (Fig. 12.1)

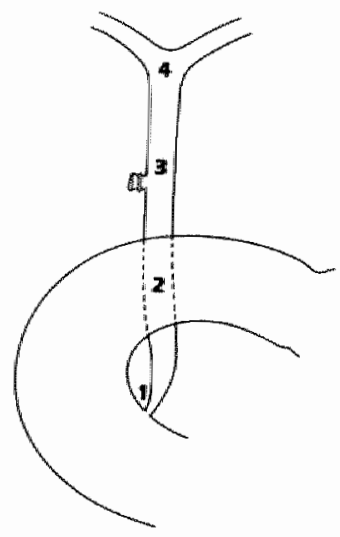

fig. 12.1: Bilio-intestinal anastomoses

Hepatico-jejunostamy (Fig. 12.1:4) is not indicated in stone disease except in case of reconstruction following stricture formation in or near the bifurcation, excision of a 
choledochocyst with or without cystolithiasis or in case of Caroli's disease. The most commonly used solutions are:

1. Sphincteroplasty

2. Choledochoduodenostomy

3. Choledochojejunostomy with a Roux-Y anastomosis

The preference in using one of these procedures depends on the personal experience of the surgeon. In the literature no indications for the individual type of bypass procedure relating to type of stones, time delay and concomitant biliary pathology can be found $(294,304,363,394)$.

There is no uniformity whether or not to use bypass surgery as an adjunct in primary CDE. Insecurity about successful lithotomy hinders accurate assessment. In view of this, the complications of a bilio-enteric bypass has to be kept in mind ("Sump syndrome "I. The excellent results on choledochoduodenostamy reported by Schein (317) are probably related to optimal CDE preceding the performance apart from the ideal and wide anastomosis, which was proclaimed as the main reason for the excellent result in this type of bypass surgery.

The late complications of bilio-enteric bypass procedures are primarily determined by the new route of bile entering the small intestine, proximal or distal from the papilla.

Duodenal bile compensates the low ph of the gastric contents passing the pylorus. In case of a proximal deviation (Fig. 12.1:3) acidification of duodenal contents, not neutralized, results in a high gastrine production (11). Continuous positive feedback consequently leads to a high gastric acid production. The development of peptic ulcer is thus explained and was already reported in 1911 11241. Pepsin inhibition due to the properties of bile salts is considerably reduced in bile diverting procedures. This must be considered of additional importance in the well established increased risk for ulceragenesis in these patients (381).

$B A$ and cholesterol absorption also may be considerably changed because the duodenum and proximal jejunum are bypassed. Passive absorption of BA mainly CDCA is suspected to be reduced. This will be compensated for by synthesis within the EHC and will probably not be of clinical importance although colonic BA concentration on the long run may be raised with known complications /Chapter 2.11).

Bacterial overgrowth in the biliary system rapidly will cause $B A$ deconjugation. Free $B A$ within the biliary system can damage the epithelium, especially the dihydroxy $\mathrm{BA}$. The villi of the enteric epithelium are most vulnarable. Resorption of lipid and lipoid substances is reduced and steatorrhoe may result.

The development of cancer in an anastomosis is only incidentally reported (333) and so far has not been recognized as a serious late complication comparable to stoma carcinoma following gastrectomy with $\mathrm{BI}$ or Bil anastomosis.

In choledochoduodenostomy gastric bile reflux is a serious thread to the gastric mucosal barrier. Gastritis results and may be so disturbing that some patients will require diversion by means of RouX $Y$ C choledochojejunostomy (175). Even after cholecystectomy this severe clinical entity has been reported (401), when bile is 
continuously draining into the duodenum, although the sphincter function of the pylorus is preserved.

In choledochoduodenostomy the diameter of the stoma seems critical in causing postoperative cholangitis (131). Both residual stone/s) and acquired choledocholithiasis are supposed to be responsible for ascending cholangitis. Tran Ky (386) reported postoperative ascending cholangitis in patients with a choledochoduodenostomy with a widle $2 \mathrm{~cm}$ anastomosis. Stoma patency thus seems no absolute guarantee to prevent this complication. Functional stoma patency can be properly assessed by isotope scanning (294). Akiyama (9) performed an endoscopic study of the upper gastrointestinal tract in patients with choledochoduodenostomy 1-5 years postoperatively. In 9 of 15 patients investigated extensive gastric-duodenal lesions were noted from mild duodenitis to severe ulceration caused by bile regurgitation. These findings have been confirmed by Rösch [299).

In cases of sphincteroplasty the main complication is pancreatitis $(16,17,165)$. Narrowing of the enlarged opening determines the ultimate result [27]. The late results of papilloplasty, if proper patient selection is maintained, are good. In general, a primary CDE has not to be followed by a bilio-enteric anastomosis (123). Neither age nor the diameter of the CBD per se is an indication for a bile diverting procedure. A bilio-enteric anastomosis is no alternative for suspected or known incomplete stone removal. The CBD should rather be cleared from residuals (stones) either by surgery or non-invasive stone extraction.

Complete, properly performed, initial surgery supported by good operative cholangiography and cholangioscopy should, in the majority of cases, eliminate the need for biliary-enteric bypass procedures.

Moreover, reviewing short and long term complications following bilio- enteric anastomosis the indications should be carefully evaluated, because cholesterol stone disease itself has a good prognosis when treated in an early stage using the proper techniques [Chapter 9 en 10 ]. 


\section{Part III}

Investigation of surgical treatment and bile composition in choledocholithiasis 


\section{Chapter 13}

\section{Results of Common Bile Duct Exploration}

\subsection{Introduction}

Ever since the very first biliary explorations were performed the clinical problem of retained stones is well known. Although intra-operative cholangiography was instituted half a century ago and cholangioscopy was introduced as a routine operative procedure almost 30 years ago, the total number of R.S. did not considerably change during this timespan because of a generalized persistent surgical reluctance to incorporate these basic techniques in routine biliary surgery. These procedures however are the main therapeutic tools to prevent R.S. Which will reduce the overall morbidity and mortality as well in surgical treatment of the gallstone patient.

In the surgical department of the De Wever Hospital in Heerlen cholangioscopy was introduced in 1976. Until July 1977 the apparatus was clinically tested. During this period of introduction the OR personnel was instructed to get familiar with the operative technique and adjunctive procedures like maintenance and handling of the scopes plus accessories.

From the first of July 1977 this operative technique was used routinely in every CDE according to a strict protocol. This clinical study only concerns the results of surgical treatment of CDS. The significance of this technique in tumor surgery will only be incidentally reported to underline the importance of cholangioscopy.

Biliary endoscopy was performed by every operator, resident and staff surgeon alike. The overall endoscopic experience within the surgical group remained constant throughout the period of study mainly because of the rotation of surgical residents. Moreover, the promotion of biliary endoscopy in surgical training cannot be substantiated additionally to the ultimate surgical results of this study. This should be kept in mind in reviewing the surgical results in treatment of CDS to be presented in the next chapters.

\subsection{Patients and Methods}

All patients undergoing biliary surgery for stone disease were included in this study during the last 5 yrs \& Jan. 1976 - 1 July 1981).

Routine cholangioscopy was started 1 July 1977. A four year experience with operative cholangioscopy will be presented.

The preceding 1 years without biliary endoscopy will act as a control period.

No other intra-operative technique, instruments or tactics were changed or added but the intraduction of the cholangioscope from 1 July 1977 onwards.

Intra-operative cholangiography was performed with a mobile $x$ - ray apparatus model Philips (BV 22) using fluoroscopy.

The contrast material used was Urombrine ${ }^{\text {. }}$

If cholangiography was combined with operative manometry the pressure reading had to be corrected with the specific gravity of the dye $[1.16 \mathrm{gr} / \mathrm{L})$. 
The apparatus used was the Caroli manometry set.

The instruments and order of sequence in instrumentation were standardized isee Chapter 10!.

\section{Protocol CDE:}

1. Operative cholanchiography (via cannulated cystic duct) combined with manometry:

2. Extraction of /visualized/ stones;

3. Endoscopic inspection $\$$ extraction of residual stones);

4. (intraoperative) T-tube cholangiography.

Commentary:

-During every cholecystectomy a peroperative cholangiogram is made to establish the indication for CDE regardiess of the preoperative criteria present. Also preoperative retro- or orthograde visualisation of the biliary system via ERC or PTC respectively is no excuse to abandon operative cholangiography because the position of CDS may be changed during the time preceding surgery.

If cannulation of the cystic duct remains unsuccessful with the regular Champeau or Berci-catheter, a puncture cholangiogram should be made. The risk for postoperative bile leakage or stricture of the CBD can be minumized using the Berci puncture cholangiogram clamp (see fig. 9.4). This strategy is still justified by the high percentage of unsuspected stones during routine cholangiography in uncomplicated gallstone disease.

With operative radiology the total number and the exact location of the CDS can be established before the CBD is explored.

A blind and lengthy instrumentation is thus avoided reducing the operative risk to the CBD.

-In most patients this technique has been combined with manometry.
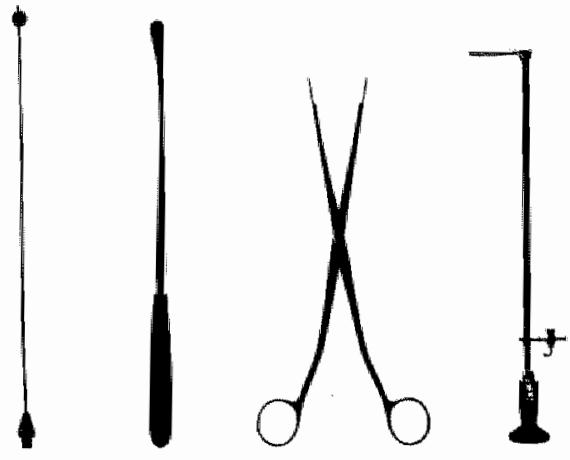

fig. 13.1

The order of sequence of the recommanded instruments used for stone extraction are illustrated in fig. 13.1.

-Cholangiloscopy is performed following stone extraction. 
Routine endoscopic inspection is outlined in Chapter 10.

If present retained stones are retrieved. The choledochotomy is sutured over an indwelling T-tube through which an operative control cholangiogram is made to outrule any residual stone, possibly missed during endoscopy.

The indication to explore the $\mathrm{CBD}$ depends primarily on the intraoperative cholangiogram. Manometry was regarded as an additional technique to cholangiography.

If the clinical indication to perform CDE could not be approved by operative cholangiography, CDE was started immediately with endoscopic inspection instead of blind instrumentation.

\subsection{Results}

The age and sex distribution of the patients are summarized in fig. 13.2. The overall sex ratio is $2.7: 1(=9: 0)$ and remains almost equal throughout the fourth till seventh decade of life. In the second and third decade the female preponderance in gallstone disease is more pronounced. The ratio is $7.5: 1$ and $4.4: 1$ respectively. The average percentage of $C D E$ related to the total number of simple cholecystectomy is $16.9 \%$. This means in one out of every six cholecystectomies the CBD was explored. This percentage increases steadily from $8.6 \%$ to $38 \%$ from the fourth till eighth decade while in the older age groups adult males have a slightly higher incidence of CDS $1 \%$ CDE: $35.7 \%$ versus $31.3 \%$ in the seventh and $50 \%$ versus $30 \%$ in the eighth decade of life; fig. 13.2).

In this study the percentage of positive CDE was $62.8 \%$. This means that in $10 \%$ of all gallstone patients CDS can be expected which is almost $33 \%$ in a patient older than 80 years. In the years investigated the total number of operations did not change. Only the number of reexplorations decreased after the introduction of the cholangioscope which parallels the decline in RS percentage (table 13.1). 

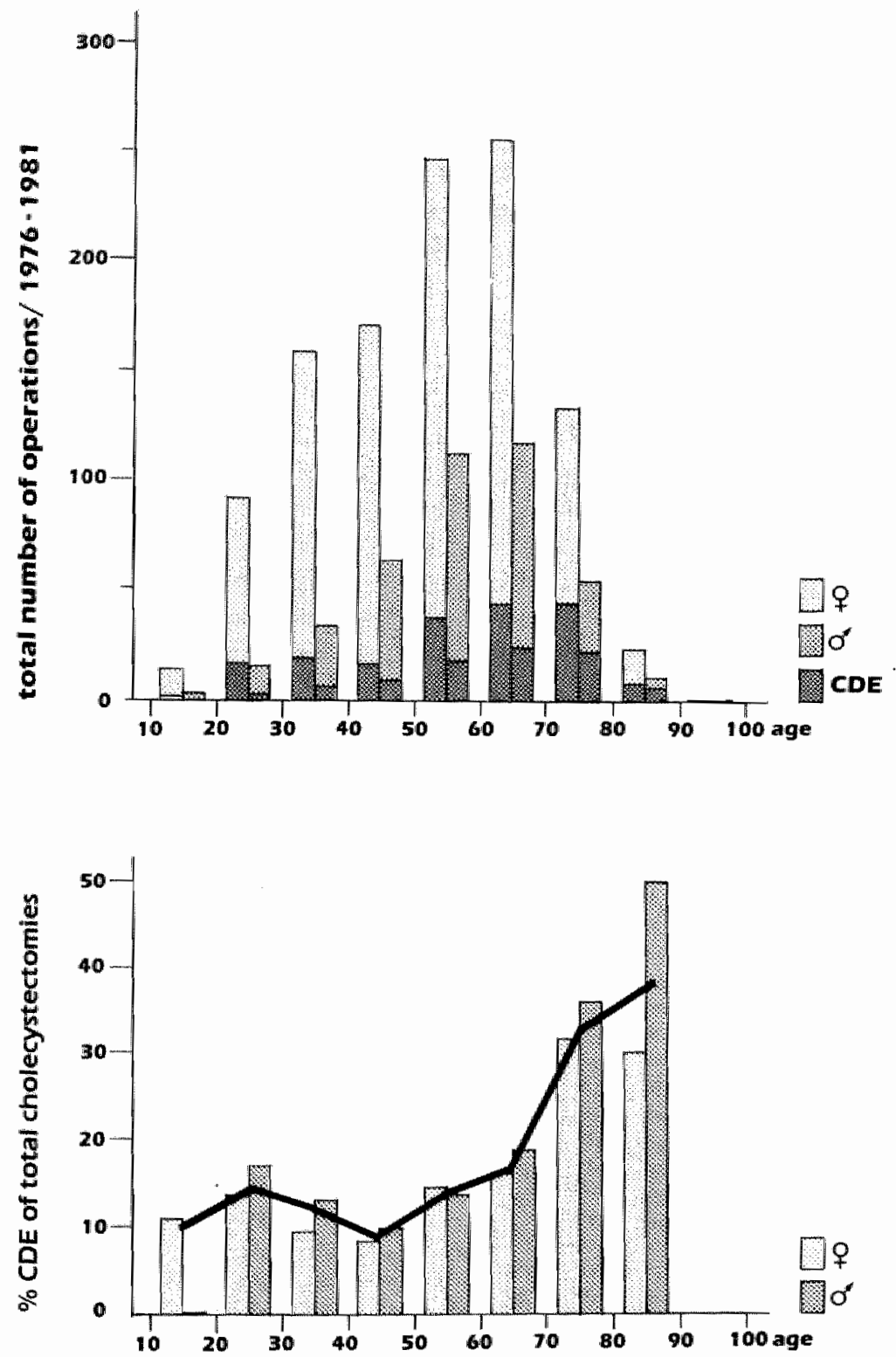

fig. 13.2 


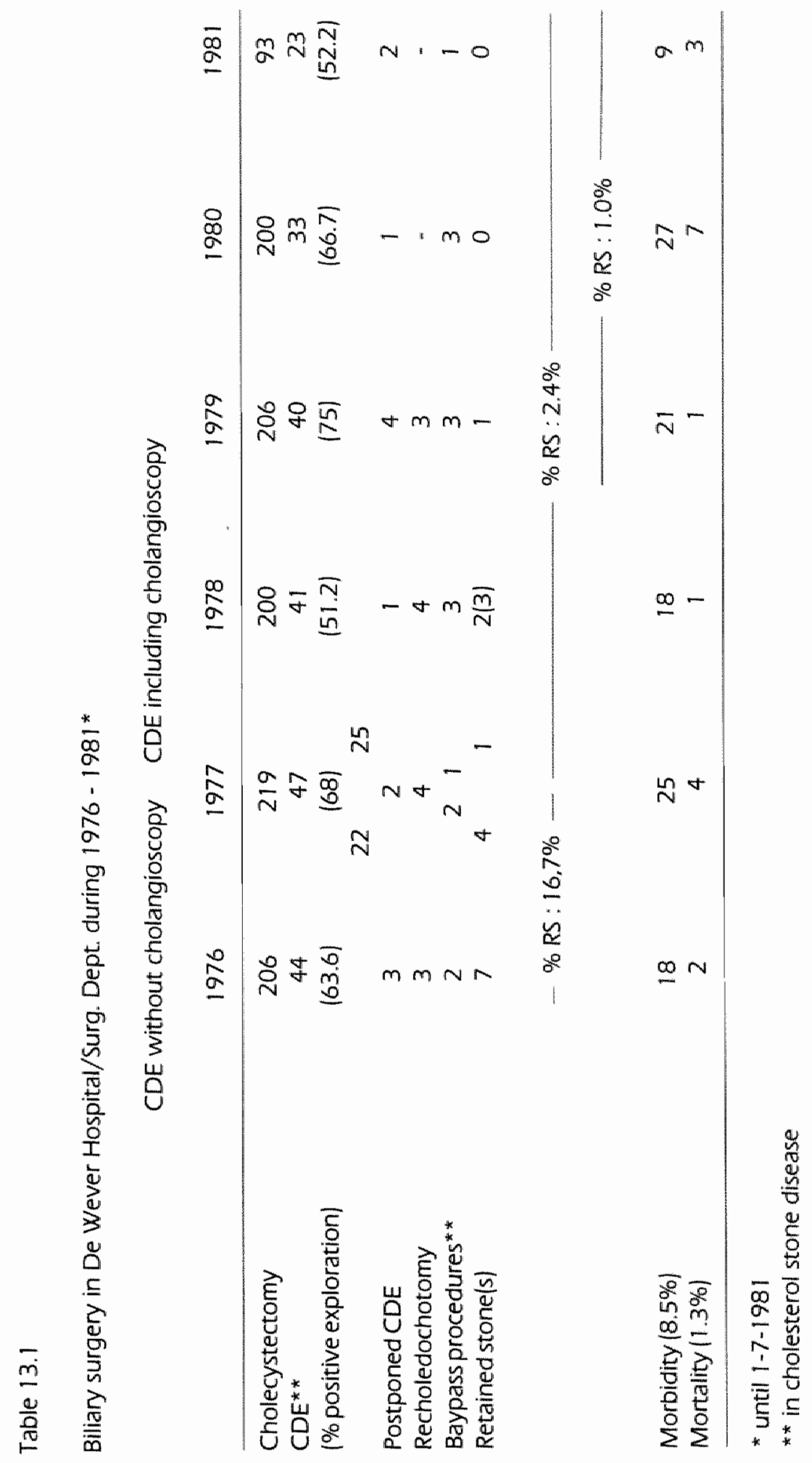


The number of postponed CDE remained constant over the years of study. This procedure is not influenced by the introduction of the cholangioscope because these operations concern patients with a long history of previous biliary surgery. The number of bypass operations per year before and after the introduction of the cholangioscope was not changed. A detailed analysis on bypass surgery in stone disease will be presented in chapter 13.6.

\subsection{Retained-Stones}

The most important finding was the reduction of retained stones in all biliary operations. In the short period preceding the introduction of routine biliary endoscopy retained stones were a very common complication following CDE 111 RS in $66 \mathrm{CDE}$, with severe postoperative sequelae.

In the period following the introduction of the cholangioscope the overall RS percentage is $2.4 \%$ of all CDE performed. This figure is further reduced to a total number of 1 RS during the last 2.5 years of biliary surgery being a RS $\%$ of $1.0 \%$. This is mainly caused by a lack of endoscopic experience of the surgeon which is clearly shown in the following case reports:

Case reports:

$1-77:^{\circ} 75 \mathrm{yr}$, underwent a difficult cholecystectomy because of severe inflammation. CDS were palpable and retracted.

A very enthuslastic operation report was made thereafter concerning the operator's first endoscopic exploration. However, 2 retained stones were shown on the postop. T drain cholangiogram who were extracted 2 weeks later via a re-exploration. The operator was probably too amazed of the new endoscopic view diverting his attention for what he had to look after.

2-78: of $58 \mathrm{yr}$., was operated in 1978 for a chronic inflamed gallbladder with CDS. Severe inflamatory reaction hindered optimal exploration of Calot's triangle. CDE was completed with cholangioscopy and T-drain cholangiograms twice with no R.S. One year later he was readmitted for a severe cholangitis. After optimal preoperative rehabilitation he was reoperated on. A cystic duct remnant or incomplete resection of Hartmann's pouch was found with severe local inflammatory reaction. Different CDS were retrieved. The interior of the biliary system did not show extensive cholangitic reaction. To guarantee optimal bile drainage a Roux-Y anastomosis was performed with an uneventful postoperative recovery. 3 - 78:? 71 yr. was operated on for CDS. An initial operative cholangiogram showed stones in the proximal system and a long cystic duct entering the CBD just proximal to the papilla. Stones were extracted and with the cholangloscope an additional proximal stone was found.

T-tube cholanglogram showed a clear system but stones in the long cystic duct remnant. The operation was terminated prematurely. During reexploration two weeks later these stones were extracted.

4. 78: see Mortality (7:1978).

$5-79: 855$ yr. underwent excision of a severly chronically inflamed gallbladder. The CBD was widened with multiple palpable stones.

CDE was performed. Cholangioscopic examination showed a severe web cholangitis which was hindering optimal inspection especially of the distel CBD. A postop $T$-tube cholangiogram showed retained stones. Two weeks later a recholedochotomy was performed and al distal stone extracted. Now a clear view could be obtained of a functioning papill. The cholangitic reaction of the CBD wall had vanished in the meantime promoting optimal endoscopic inspection this time. The operator's endoscopic experience was less than 1 year. 


\begin{abstract}
Analysis:
In case report 5 optimal endoscopic inspection was severly hindered by an extensive web cholangitis in which multiple stones were located. The severe cholangitis was. hindering optimal inspection. This case report clearly shows that without any information on the papil obtained during clear inspection with the cholangioscope * the interpretation of the postexploration T drain cholangiogram is very difficult. In fact, the operative strategy combined with incomplete endoscopic experience contributed to the existence of the R.S. postoperatively and should not be regarded a failure of the technique per se.

In case reports 2 and 3 choledochoscopic inspection during the first CDE was negative. The retained stones in these patients are also rather a complication of a failed operative technique than a failure rate attributable to biliary endoscopy. Case report 4 is also not a complication due to cholangioscopy but rather a wrong operative strategy during the first operation by abandoning routine cholangiography because of failure of cannulating the cystic duct, which resulted in a lethal outcome 2 years later. This case report stresses the importance of operative cholangiography whether by cannulation or puncture of the CBD. Although this patient did show retained stones cholangioscopy cannot be blamed for this lethal complication.
\end{abstract}

Case report 1 very clearly shows the problem of failure rates by lack of experience and also demonstrates the importance of a double control method in CDE by way of routine $T$ drain cholangiography following completion of $C D E$.

Disregarding the RS reported in case reports 2,3 and 4 , only 2 RS can be attributed to failure of the technique of cholangioscopy but are both rather related to inexperience with the technique than to billiary endoscopy per se.

\title{
13.5 Complications
}

The overall morbidity in Billiary Surgery during the period of investigation is rather high $8.5 \%$; (see table 13.1). A critical analysis of all complications is shown in table 13.2 .

In most series only the septic complications are mentioned.

In this study all complications are listed and divided in patient and operation-related complications. 
Table 13.2

Complication in Biliary surgery during $1976-1981$

\begin{tabular}{|c|c|c|c|c|c|c|}
\hline Complications: & 1976 & 1977 & 1978 & 1979 & 1980 & 1981 \\
\hline \multicolumn{7}{|l|}{ I Patient related } \\
\hline 1. Pulm. infection & $2 / 2$ & $2 /-$ & & $1 / 3$ & $3 / 1$ & $1 /-$ \\
\hline 2. Urinary infection & & $-/ 1$ & $-1 / 2$ & -13 & $3 /-$ & \\
\hline 3. Thrombo-embolic compl. & $-/ 1$ & $2 / m$ & $-/ 1$ & $\alpha / 1$ & & \\
\hline$\|$ Operation related & & & & & $2 / 1$ & $-/ 2$ \\
\hline 5. Wound infection & $2 / 2$ & $5 / 2$ & $3 / 2$ & $5 / 2$ & $9 / 3$ & $2 / 2$ \\
\hline 6. Wound dehiscence & & & & $-\gamma$ & & \\
\hline 7. Sepsis & & & & & $1 / \mathbf{-}^{*}$ & \\
\hline 8. Intra-abd. infection & & & $-/ 1$ & -11 & & \\
\hline 9. Cholangitis & -11 & & & & & \\
\hline 10. T-drain channel infection & & & $-/ 2$ & $-/ 2$ & & \\
\hline 11. Bile leakage & & $3 / 1$ & & $-/ 1$ & $-/ 3$ & $1 / \%$ \\
\hline 12. Haemorrhage (intra-abd.) & & $2 /-$ & $1 / 1$ & & & \\
\hline 13. Gastro-intest. haemorr. & & & -11 & & & \\
\hline 14. Enteric fistula & & $1 / \%$ & & & & \\
\hline 15. Pancreatitis & & & $-/ 1$ & & & -11 \\
\hline 16. latrogenic laesion & $1 /-$ & & $-/ 1$ & $1 /-$ & $1 /-$ & $1 /$ \\
\hline 17. Retained stone [s] & $1 / 6$ & $-/ 5$ & $1 / 2$ & $-/ 1$ & $-\gamma-$ & $\%$ \\
\hline Total (cholecystectomy/CDE) & $18|8 / 10|$ & $25 \mid 16 / 9 !$ & $18(4 / 14)$ & $|2||6 / 15\rangle$ & $27(19 / 8)$ & $10(5 / 5)$ \\
\hline
\end{tabular}

* Sepsis due to simultaneously performed colon surgery

As is clearly shown in the case reports on mortality chapter 13.6,

CDE exploration considerably increases the overall complication rates.

Re-exploration is a very hazardous operation but mostly come about by the type of patient rather than the type of operation performed.

The excuse of abandoning CDE because of an increase in operative risk is only valid in aged patients; most older patients die of cardio-vascular problems rather than of operation related complications.

The total complication ratio's did not change in the time period before and after the introduction of the cholangioscope in routine biliary surgery. Especially the septic complications in CDE did not increase. 
The iatrogenic leasions to the CBD mostly concerns laceration of cystic duct or partial incision in a severely inflamed hepato-duodenall ligament. The only complication directly attributable to the cholangioscope was a short laceration of the choledochotomy which could be repaired without sequelae.

The complications directly associated with T-tube drainage are:

T-drain channel infection ( $N$ : 4 patients). Bile leakage has been noted different times during the years of study with a lethal outcome in 2 patients. T-tube extraction should not be performed unless 2 weeks postoperatively. If done earlier the tube channel can be ruptured during extraction. Actually, if T-tube extraction is routinely followed by fistulography within one week postoperatively, a high percentage of leakage can be expected. This is however no argument in favour of persistent drainage during an indwelling T-tube because 2 drains in 1 area have an eroding effect on each other, preventing optimal fistula forming.

If a CDE is drained it should be done whether by internal splinting or optimal subhepatic drainage but 2 drains lasting for more than 2 days in close proximity are dangerous and should be condamned.

\subsection{Mortality in Biliary Surgery}

\section{Calse reports:}

$1(1976)$ o $74 \mathrm{yr}$.

$2(1976)$ of $87 \mathrm{yr}$

$3 \| 1977)$ \& $71 \mathrm{yr}$

$4(1977)$ of $43 y r_{0}$

$5119771973 \mathrm{yr}$

$6(1977) 968 \mathrm{yr}$

$7(1978) d 69 \mathrm{yr}$

$8(1979) 982 \mathrm{yr}$
Who underwent cholecystectomy and CDE following a acute cholecystitis. Founteen days postoperative he was re-operat ted for retained stones. The first postop. day he died of massive pulmonary emboli.

died 4th postop. day during severe cholangitis following cholecystectomy and CDE. Post mortem examination nor postop.

Tutube cholangiogram was done but retained stones semed hiost likely the cause of death.

died 4 th postop. day of severe peritonitis due to bile leakage from the cystic duct.

died 4th postop. day of bile peritonitis caused by frank bile leakage from the liverbed which apparantly was inefficiently ctrained. Relaparotomy could not restore the patient"s general condition. died 2 th postop. day of septic shock caused by ruptured subphrenic abcess following acute cholecystitis. Post mortem examination showed a galloladder carcinoma which was cured by resection showing no infiltration in the gallbladderwall.

died 2 th postop. day of sepsis following emergency cholecystectomy because of gallbladder pertoration during conservative treatiment of severe cholecystitis.

died 14 th postop. day of severe pancreatic necrosis following bind intraductal instrumentation resulting in a fausse route. Internal drainage following repair failled to cure the patient. died 3th postop. day of acute cardiac arrest due to hearttamponade caused by a ruptured proximal aortic syphilic aneurysm. 
$9(1980) \% 80 \mathrm{yr}$
$10(1980)+72 \mathrm{yr}$
$11(1980)$ o $56 \mathrm{yr}$
$12(1980) 980 \mathrm{yr}$
$13(1980) 962 \mathrm{yr}$
$14(1980) \approx 71 \mathrm{yr}$.

$15(1980) 379 \mathrm{yr}$

$16(1981) 989 y^{\prime}$

$17|1981| 985 \mathrm{yr}$

$18(198 \|) 67 y$ died 2 th postop. day of acute heart failure following myocardial ischaemia during cholecystectomy.

died 17 th postop. day of severe peritonitis due to bile leakage from an unsuccessful revised bilio intestinal anatomosis because of severe local inflammatory reaction.

died 35 th postop. day of sepsis by generalized billary peritonitis due to persistent bille leakage following T-tube extraction. died 7 th postop day of necrotising pancreatitis following papilloplasty because of papil disfunction died 14 th postop. day of R.D.S. due to severe therapy resistant biliary pancreatitis.

died 2 th postop. day of acute myocardial ischaemia. Previously performed cholecystectomy was done without cholangiography in 1978 .

A choledocho-duodenostomy was performed following $\mathrm{CDE}$. Besides repeated cardiac failures and cholangitis this patient also suffered from an obstructing rectal neoplasm which was simulta neously treated by decompression via a colostomy.

died 3th postop. day. Energency laparotomy fol lowing PTC was performed because of generalized biliary peritonitis. A retained stone in the distal CBD was extracted In 1973 cholecystectomy and CDE without cholangioscopy was performed. died first postop. day of cardiac failure.

Im 1960 she underwent cholecystectomy without cholangiography. A lange CDS was extracted during the postponed CDE.

died 2 th postop. day of cardiac failure. She underwent cholecystectomy in 1955 without additional peroperative diagnos tic. Three large stones were retrieved. Endoscopic view of internal biliary system was free of severe chollangitic reaction. A choledocho-duodenostomy was performed because of delayed visualisation of the duodenum.

died 2 th postop. day of severe bile leakage from the liver. following excision of a severely adherent gallbladder which appeared to be a carcinoma on postmortem examination.

In six patients a lethal outcome was promoted by the existence of Retained CDS following cholecystectomy $\pm \mathrm{CDE}$. One patient $(10)$ died of the complications due to bypass surgery.

These patients clearly show the increase in mortality by insufficiently primary operation omitting cholangiography and / or cholangioscopyl

\subsection{Bypass Surgery}

In the period of study in which cholangioscopy was performed regularly the total number of bypass operations performed in stone disease decreased (Table 13.1). A short case history of each patient is presented. In three patients the operation was followed by a lethal outcome. One patient died of non-operation related factors (10-1981). In only one patient (8-1980) the indication to do a bypass operation can be questioned retrospectively. 


\section{Case histories:}

$\|-1977$ o 81 yr.

$2 \times 1978$

3. -1978 \& $29 y \mathrm{r}$

$4-1979+80 y r$.

5-1979? 75 yr.

$6-1979 \% 66 y^{\prime}$

$7-1980$ q 73 yr.

B-1980 $53 y /$

$9-1980 \div 65 \mathrm{yr}$

10-1981 $85 \mathrm{yr}$, 11-1981 q $71 \mathrm{yr}$. had progressive jaundice caused by distal CBD stenosis seen on

PTC. During inspection the pancreas was normat on palpation. Papilla was inspected via a duadenctomy. A clear view of the papll could not be obtained with the cholangioscope because of a severe ectasia of the CBD. By exclusion the diagnosis vas proposed to be a papillitis stenosans. A choledochoduodenostomy was performed. No stones wiere found. See mortality 1978.

with jaundice by an unknown process in the pancreatic head obstructing the distal CBD. No stones were found. A choledochoduodenostomy was performed.

who appeared to have allarge choledochocele during billary exploration which was excised and coexistent calcull retrieved. An end to end cholledochojejunostomy was performed to restore bile flow.

with multiple stones in the CBD. A stone in the LHD could not be retrieved even using the chollangloscope. A permanent Roux-Yanastomosis for permanent biliary drainage was performed. underwent a cholecystectomy in 1978 without cholangiography. Multiple stones and mud were extracted from the billiary system this time. A coexistent duodenal diverticulum was discovered on operative cholangiography which interfered with bille flow. This was excised and a choledochaduodenostomy was performed. was admitted for acute cholecystitis plus CDS.

After $C D E$ incrustated stone fragments remained in the distal $C B D$ : a choledochoduodenostomy was performed. The sixth postop. day a sudden frank bile leakage was noted wia the drain and ielaporotomy was performed. The loosened anastomosis was repaired by way a Roux-Y choledochojejunostomy. Seven days later she died of bile peritanitis due to recurrent bile leakage.

underwent a cholecystectomy in 1955 followed by a delayed CDE 5 years later. She had episodes of fever with mild jaundice due to a large retained earthy stone which was extracted. Art additional Roux-Y anastomosis was performed.

who underwent cholecystectomy and CDE. A large stone in the LHD could not be extracted. A permanent drainage by a Roux-Y anastomosis was performed.

see mortality $(17-1981)$

underwent cholecystectomy and CDE. No CDS were found. Post exploration T-tube cholanglogram showed no dye entering the duodenum while with the scope in the distal $C B D$ different small papillomas were seen. A duodenotomy plus papillotomy was pierformed and different papillomata excised. A papilloplasty wats performed in addition.

The indications for bilio-intestinal anastomosis remain elusive in the literature especially in case of cholesterol stone disease.

All three types of bilio-enteric bypass operations have a certain ratio of morbidity and mortality as well. So proper indications have to be maintained in performing this extra surgery. In many instances it concerns old and frail patients with severe septic 
complications due to the biliary stones, already jeopardizing the general condition of these patients.

In experienced hands these operations are without any complication as shown by Schein (317) who proclaims to perform an additional choledocho-duodenostomy following every CDE for biliary stones. Especially in case of bilio-intestinal bypass procedures there exists a gradual difference between optimal and maximal surgery. The complications of this operation however should be related to the calculated risk of retained stones. This is probably the main reason why these surgical procedures are performed arbituarily: the problem of retained stones is shortcircuited by performing a bilio-intestinal anastomosis. Promoting this surgical pracedure by showing excellent results but handling undue indications, resembles tautologic argumentation and is no proof of a justifiable treatment.

Fig. 13.3: Biliary Surgery in the Netherlands during 1975-1979, with special emphasis on bilio- enteric anastomosis.

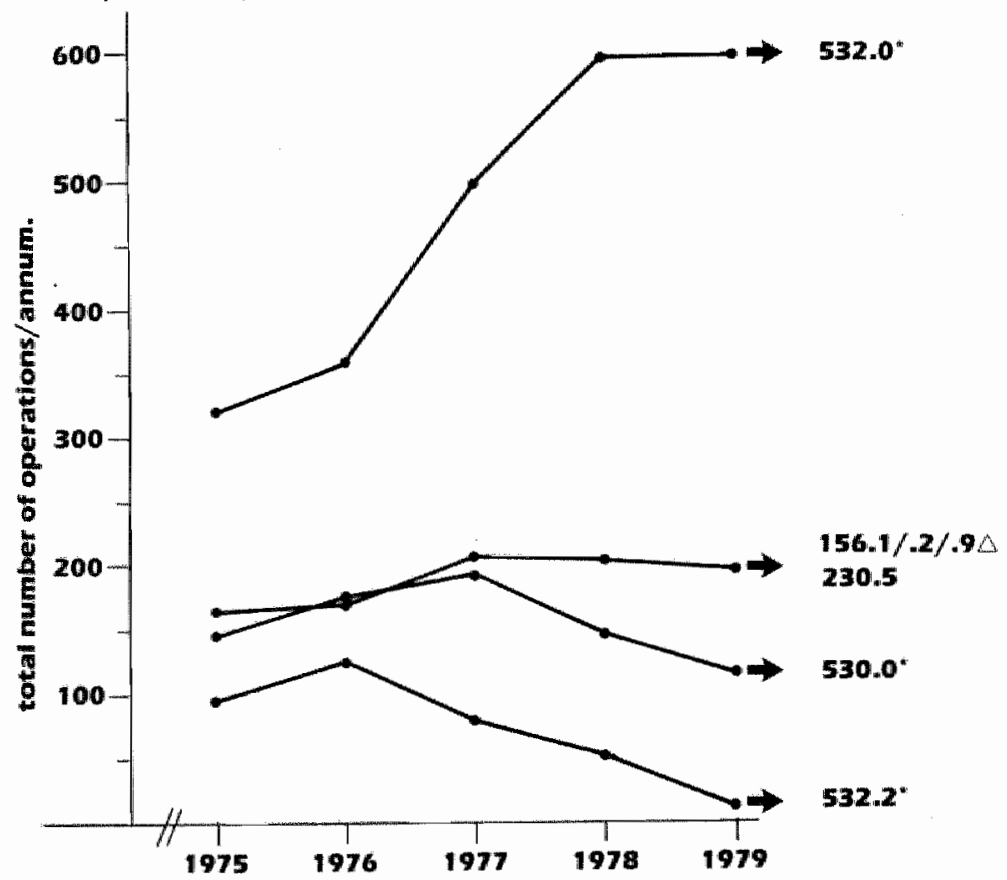

* : $532.0=$ Biliodigestive anastomosis

$530.0=$ Re-exploration

532.2 = Re-exploration with Biliodigestive anastomosis

$\Delta: 156.1$ = Carcinoma of extra hepatic biliary system

156.2 = Papil carcinoma

156.9 = Carcinoma biliary system (without specification)

230.5 = Biliary and hepatic malignancy (without specification)

The numbers refer to the classification of operations and diseases from: Stichting Medische Registratie (SMR|/Utrecht. 
This can be clearly shown in reviewing the total number of bilio-intestinal anastomoses in the Netherlands during the years 1975-1979 (fig. 13.3).

Throughout the last three years the total number of re-explorations with and without a bilio-intestinal anastomosis has steadily declined after a sharp rise initially in the preceeding years. This shows indirect but suggestive evidence the total number of retained stones has decreased. In the same period of time the total number of bilio-digestive anastomosis as a primary procedure rises almost exponentially. In the same timespan the total number of malignant diseases which are primarily treated with a primary bilio-digestive anastomosis, remained constant. From these data one can conclude that a bilio-intestinal bypass as a primary procedure must have been more frequently performed in stone disease.

There is however no need for this type of surgery in biliary stone disease because the risk for retained stones can be dramatically decreased to an absolute minimum of $0-1 \%$ as is also shown in this study.

Using routine cholangiography and cholangioscopy in every CDE the indication for bilio- intestinal anastomosis in gallstone disease can be reduced to patients with:

1 cholledochocele [both as bypass or as repair following excision);

2 located, irremovable retained stones ("intractable stone disease");

3 unknown pathology of the papillary region;

4 (localised) sclerosing cholangitis;

5 (retroperitoneal fibrosis.)

The following indications should no longer be used to perform bypass operations:

1 cholangitis;

2 multiple stones:

3 "primary", earthy stones (mud):

4 widened CBD (age matched).

\subsection{Operative manometry}

In 1976 there was a high failure rate of cystic duct cannulation $\sharp 40 \%$ ) of all cholecystectomies, so neither manometry nor cholangiography was performed. That is the reason why operative cholangiography and manometry are reviewed during the period 1977-1981. In 1977 cystic duct cannulation ratio increased, probably by the introduction of the protocol on CDE including operative cholangiography. In every CDE cholangiography was performed with the instrument of Caroli for operative biliary manometry.

The normal values are:

$15 \mathrm{~cm} \mathrm{H} \mathrm{H}_{2} \mathrm{O}$ for passage and residual pressure. All data on manometry were recalculated because of the relatively high specific gravity of the radiopaque material |chapter 13.2). 
Table 13.3

Results on manometry in CDE during $1977-1981$

\begin{tabular}{ccccc} 
& false pos. & false pos + Rö & false neg & total \\
\hline 1981 & 5 & 2 & 4 & $9(7)$ \\
1980 & 3 & 1 & 6 & $9(8)$ \\
1979 & 4 & 2 & 5 & $9(7)$ \\
1978 & 5 & 3 & 13 & $18(15)$ \\
\hdashline 1977 & 4 & 1 & 1 & $5(4)$ \\
\hline Total & 21 & 9 & 29 & $50(41)$
\end{tabular}

The results are summarized in table 13.3 .

The total number of non-informative information about the presence of CDS was very high: $50 / 162=32 \%$. In 9 patients both manometry and cholangiography gave suggestive evidence on the presence of CDS. So neither manometry nor cholangiography contributed to extra information about the excistence of CDS. The patients with a false negative result were only those patients with stones located in the distal CBD. Despite of this location the pressure failed to show any suggestive evidence on the presence of these distal stones Patients with normal pressure readings and stones in the proximal system were not included.

These reported results show manometry does not increase the accuracy rate of cholangiography. In many instances a high pressure reading cannot be sufficiently explained by additional operative findings resulting too easily in a (unduly indicated) bypass procedure.

If the accuracy rate of operative cholangiography can be increased there is no need for additional intra-operative diagnostic techniques like manometry or flow measurement anyway.

In conclusion, in a total of $32 \%$ of all patients who underwent CDE manometry was of no use in diagnosing CDS in our series. The total number of 60 negative explorations constitute a serious risk for an increase in operative morbidity. This warrants serious consideration in advocating this technique as a mandatory intraoperative diagnostic tool.

\subsection{Operative biliary radiology}

In table 13.4 the results on cholangiography are summarized.

In a review concerning all operative cholangiographies only false positive cholangiograms can be verified by a negative $C D E$.

The total number of false negative cholangiograms cannot be properly assessed immediately but will appear in the years to come when patients are readmitted with CDS. This uncertain number of patients will naturally be omitted in this evaluation on intra-operative rontgendiagnostics in CDE. 
In 31 patients operative cholangiography was indicative for the presence of stones which was supported by manometry in only 9 patients. In 31 patients the single indication to perform CDE was a false positive cholangiogram resulting in a negative CDE.

Table 13.4

Result of cholangiography in CDE during $1977-1981$

\begin{tabular}{lccc}
$\begin{array}{c}\text { false pos. Rö } \\
\text { (initial Rö) }\end{array}$ & $\begin{array}{c}\text { false pos. Rö } \\
+ \text { manometry }\end{array}$ & $\begin{array}{c}\text { false pos. Rö } \\
\text { perop. T-tube }\end{array}$ \\
\hline 1981 & 5 & 2 & 2 \\
1980 & 4 & 1 & 3 \\
1979 & 6 & 2 & - \\
1978 & 9 & 3 & 2 \\
\hdashline 1977 & 7 & 1 & 1 \\
\hline Total: & 31 & 9 & 8
\end{tabular}

The peroperative postexploration cholangiogram was suggestive for residual biliary pathology in 12 patients. In most of the patients non- visualization of the duodenum was noted because of papilla abstruction. Re-exploration was not performed because during cholangioscopy papilla function could be clearly observed. Two patients were treated before the intraduction of routine cholangioscopy in CDE but on the postoperative T-drain cholangiogram no residual stones were visualized.

During the period of routine cholangioscopy in only 1 patient a residual stone was established on the post-exploration cholangiogram which was overlooked during the preceeding cholangioscopy.

Special attention should be paid to those patients in which the initial peroperative cholangiogram was the only method demonstrating the CDS (table 13.7). This total

Table 13.7

Unsuspected CDS

\begin{tabular}{lccccc} 
& 1977 & 1978 & 1979 & 1980 & 1981 \\
\hline Total No. patients & 5 & 3 & 4 & 7 & 12 \\
No. pos. CDE & 13 & 22 & 30 & 21 & 32 \\
$\%$ Unsusp. stones & 38.5 & 13.6 & 13.4 & 30.0 & 37.5 \\
\hline
\end{tabular}

$-X \%$ unsusp. stones : $26.6 \%$

- for legend see text 
number of unsuspected stones is relatively high because no extensive radiologic examination was carried out routinely to establish CDS preoperatively. This was done because operative cholanglography is far more accurate than the preoperative IVC in diagnosing CDS. A preoperative IVC is no excuse to abandon peroperative cholangiography. Even if on an IVC CDS are proven a peroperative cholangiogram has to be performed: the location and total number of CDS has to be established exactly to carry out an optimal intraductal instrumentation with a minimum of operative risk to the bilirary system.

The results of operative cholangiography can be improved with better Röntgen outfit in the OR. The discrapency between Röntgen armentarium on the OR and the $X$-ray department is responsible for the poor results. The acknowledgement of operative radiology both by radiologist and surgeon will guarantee optimal results in operative cholangiography. The net effect will be a reduction of the total number of negative CDE resulting in a decrease in morbidity and mortality in biliary surgery.

\subsection{Results of Cholangioscopy}

In all patients CDE was completed with cholangioscopy except in 2 patients in which the choledochoscope was not available because CDE was simulteneously performed in 2 OR. The total number of CDS which are removed before and after routine cholangioscopy are summarized in table 13.6 and 13.5 .

Table 13.6

Total number and location of CDS found before cholangioascopy

\begin{tabular}{lrrrrrr}
\hline & & 1977 & 1978 & 1979 & 1980 & 1981 \\
\hline 6 Location: & - & - & - & 5 & 2 & 2 \\
5 & - & 4 & 15 & 12 & 6 \\
4 & - & 20 & 16 & 24 & 8 \\
3 & 9 & 5 & 18 & 16 & 8 \\
2 & 9 & 9 & 9 & 5 & 6
\end{tabular}

Table 13.5

Total number and location of found with cholangioscopy

\begin{tabular}{|c|c|c|c|c|c|c|}
\hline & & 1977 & 1978 & 1979 & 1980 & 1981 \\
\hline 6Location: & & - & 1 & 5 & 1 & 1 \\
\hline 5 & $x$ & - & - & 3 & - & - \\
\hline 4 & +5 & 2 & 2 & 6 & 2 & - \\
\hline 3 & $5^{x}$ & - & 2 & - & - & - \\
\hline 2 & $t$ & 1 & 4 & 5 & 5 & 1 \\
\hline 1 & $2^{2}$ & 2 & 7 & 6 & 4 & 2 \\
\hline total no. patients & & 5 & 12 & 10 & 10 & 3 \\
\hline 104 & & & & & & \\
\hline
\end{tabular}


In the proximal and distal biliary system most stones were left following blind instrumentation which were then discovered during cholangioscopy. In several patients proximal stones were localized with the cholangioscope after extraction of distal stones. These might have been migrated or not properly visualized on the initial cholangiogram.

This high number of residual stones before cholangioscopy could have been reduced by extended blind instrumentation. This increases the risk for iatrogenic leasions to the interior of the biliary system which can easily result in misrepresentations on post-exploration T-tube cholangiograms. In this study all negative explorations could be supported by cholangioscopic inspection.

in different patients the post-exploration T-tube cholangiogram showed no duodenal visualization but preceeding cholangioscopic inspection, showing proper papilla function, could avoid re-exploration. In this respect cholangioscopy can be regarded a supplementory diagnostic aid in case of difficult cholangiographic appearances.

In 5 patients intraductal instrumentation was done under visual control using the cholangioscope, but in I patient stone extraction failed and a Roux- $Y$ anastomosis was performed (13.7: Bypass Surgery case history 5-1979).

In 4 patients a red papilla was found suggestive of a recent stone passage and /or stone impaction.

Other types of pathology were found during cholangioscopy. In 3 patients a single papilloma was located in the distal CBD. Histologic examination of the biopsy taken with the scope showed no malignancy.

In 1 patient a papillarycarcinoma could be established by biopsy via the scope which impressed an incrustated papil stone on PTC. A Whipple operation was performed after 2 weeks of biliary decompression.

One male patient with severe icterus had a double tumor shown on PTC, one at the bifurcation and the other in the pancreatic region of the CBD. A choledochotomy was performed. The distal tumor must be of pancreatic origin because endoscopically the mucosa was intact and external compression could be clearly seen. The proximal tumor appeared to be the primary leasion: a cholanglocarcinoma with an intra-pancreatic metastasis.

In summary, endoscopic inspection guarantees a complete lithotomy.

Papilla function can be properly visualized and be differentiated between organic and / or functional stenosis.

Papilla dilatation has thus become an useless and dangerous procedure, promoting papil fabrosis.

Additional pathology can be detected.

In cancer surgery the extension and exact lacation of the maliqnancy can be determined. A biopsy can be taken under visual control and surqery planned.

Careful blind instrumentation preceeding cholangioscopy guarantees a minimum of wall damage, especially in the distal CBD. This reduces an unnecessary and lengthy instrumentation reducing the operative risk to the benefit of the patient. 


\subsection{Conclusion}

In this study operative cholanqiography and cholangioscopy were included in a prospective protocol of $\mathrm{CDE}$. The aim was to minimize blind intraductal instrumentation with a small risk of iatrogemic damage.

Cholangioscopy could locate different stones irrespective of the location of previous removed stones. In only one patient the T-tube cholangiogram showed a residual stone following cholangioscopy. But in this patient no proper visualization could be obtained of the distal CBD. The papilla was not seen.

The value of operative T-tube cholangiography seems at least questionable if the surgeon is familiar with biliary endoscopy.

The value of intra-operative manometry as an adjunct to cholangiography must be re-evaluated but with the results shown in this chapter the technique does not add any extra information on the presence of CDS in gallstone patients. The high number of false positive results resulting in negative $C D E$, may unduly increase the morbidity ratio in biliary surgery.

Operative radiology can be improved with sophisticated $X$-ray equipment already present on most $X$-ray departments.

Both cholangiography and cholangioscopy are indispensible in biliary surgery and should be performed routinely without reservation. 


\section{CHAPTER 14}

\section{The composition of hepatic bile in choledocholithiasis}

\subsection{Introduction}

Although the tatal number of cholecystectomies performed in the U.S. alone exceeds half a million, there are no accurate data as yet available to differentiate primary cholesterol CDS by lack of physiological and chemical data on stone formation and bile lipid composition following cholecystectomy completed with CDE.

This limits the accuracy of the discussion on the topic of reforming biliary cholesterol stones. A possible change in lithogenecity might be a factor either in favor of or against continuous stone formation in the absence of the gallbladder in the postcholecystectomy patient. W/ide variation in treatment, especially the need for internal drainage at primary exploration as an adjunct in surgical therapy of CDS will thus prevail.

The purpose of the present study was:

1. to look for any difference in bile lipid composition between gallstone patients with and without coexistent CDS which might explain stone formation in the biliary system outside the gallbladder $1=$ primary cholesterol CDS);

2. to investigate the difference in per- and postoperative bile lipid composition in patients with CDS whichis despite appropriate treatment, responsible for a persistent risk of newly formed stones.

If lithogenic bile is highly susceptible in the postoperative phase following cholecystectomy the prognosis of the operated CDS patients should then be reevaluated and the indication of bilio-intestinal anastomosis as a primary procedure should be considered.

As previously explained in chapter 2.1 total BA and cholesteral concentration and the four major BA are established rather than estimating one of the numerical or graphic representations in use. Methodology of determination of the different bile lipid components varies between laboratories. Also errors in estimation of the individual components can be masked in calculating one of the lithogenic indices. This can be met by estimating cholesterol saturation in vitro in the clinical laboratories dealing with BA chemistry, thus minimizing standard errors in calculating the different indices.

However, changes in the individual bile lipid components seem more rellable as will be shown in this study. Also the relative concentration of the individual BA will be established which may be helpful in explaining some metabolic changes in the EHC of BA after cholecystectomy.

\subsection{Patient selection}

In this study 19 patients with proven CDS were included. There was no difference in age, sex and duration of symptoms with other patients operated on for CDS.

A group of 19 patients was selected on whom simple cholecystectomy was 
performed. These patients had no history of jaundice or other symptoms suspecting CDS, no history of (sub-) acute cholecystitis of other severe complications of cholecystolithiasis necessitating hospitalization.

In all patients peroperative cholangiography was performed to exclude CDS finally. These patients with uncomplicated cholecystolithiasis served as a control group. In 5 patients approved consent was obtained to examine late postoperative bile lipid composition. Bille was sampled by duodenal aspiration. These samples were taken from 4 till 18 months postoperatively.

\subsection{Methodology of bile sampling}

\section{Intra-operative sampling:}

During cholecystectomy a minimum of $2 \mathrm{ml}$ of hepatic bile was aspirated via a canula in the cystic duct prior to peroperative cholangiography. This was done because bile sampling following peroperative cholangiography and/or CDE will undoubtedly lead to inaccurate and incomparable data on bile lipid composition because:

- all radiopaque substances have a strong choleric effect (84) and up to $30 \%$ will remain in the EHC for more than 3 days;

- intraductal instrumentation includes abundant rinsing which causes dilution of bile;

- a choledochotomy should be regarded as a fistula on the EHC which interferes with bile lipid composition.

2. Postoperative sampling:

The second bile sample is taken postoperatively before extraction of the T-tube. Sampling conditions are:

- a negative postoperative T-tube cholangiogram with regard to retained stones.

- T-tube should be continuously clamped during 24 hours. - patients must be fasting.

3. Late postoperative sampling:

In the fasting patients a silastic tube is situated in the duodenum near the papilla. This is radiologically controled if needed. No stimulants are used to increase bile flow because these drugs llike MgSO4 | may enhance intestinal motility, influencing bile lipid composition.

Nitroglyceride should be preferred in duodenal bile sampling because this only relaxes the sphincter without any effect on bile flow and bile lipid composition. However, in none of our patients medical stimulation was necessary to obtain adequate samples.

\subsection{Laboratory methods}

a Reagents used:

- Sodium acetate buffer $0.1 \mathrm{~N}, \mathrm{pH} 5.6$.

- N-cholylglycine hydrolase [Schwarz-Mann, 10,000 Units:ml]. - Ethyl acetate. 
- Hydrochloric acid $6 \mathrm{~N}$.

- Bile acid standard: approximately $20 \mathrm{mg}$ each of the bile acids cholic acid, deoxycholic acid, chenadeoxycholic acid and litho cholic acid are dissolved together in chloroform containing $5 \%$ methanol, total volume $100 \mathrm{mll}$.

- Internal standard: $50 \mathrm{mg}$ of 7-ketodeoxycholic acid is dis solved in chloroform (containing $5 \%$ methanol), total volume $100 \mathrm{ml}$.

- 1,1,1,3,3,3-hexafluoroisopropanol (HFIP, Pierce chemical no. 23950).

- Trifluoroacetic anhydride (TFAA, Pierce chemical no. 67363).

- Acetonitrile.

b. Procedure:

Hydrolysis and extraction:

Two $\mathrm{ml}$ of internal standard solution is evaporated to dryness in a $50 \mathrm{ml}$ tube and then $0.1 \mathrm{ml}$ of bile, $2.5 \mathrm{ml}$ of acetate buffer and 500 units of enzyme are added. The mixture is incubated during 3 hours at $37^{\circ} \mathrm{C}$.

The sollution is then extracted once with $20 \mathrm{ml}$ of ethyl acetate for 10 minutes with shaking. The tubes are centrifuged and the water layer is discarded by suction. The organic layer is dried with sodium sulphate and, after filtration, evaporated to dryness in vacuo at $40^{\circ} \mathrm{C}$.

Derivatisation and gas chromatography:

A standard is prepared by adding $0.5 \mathrm{ml}$ of internal standard solution to $2 \mathrm{ml}$ of mixed $B A$ standard solution.

The combined solution is evaporated to dryness and the residue treated in the same way as in the sample tubes. To the dried residue in the tubes $0.1 \mathrm{ml} \mathrm{HFIP}$ and $0.2 \mathrm{ml}$ TFAA are added, the mixure incubated for one hour at $37^{\circ} \mathrm{C}$ and afterwards dried in a nitrogen stream. The residue is then dissolved in $0.2 \mathrm{ml}$ of acetonitrile and gas chromatography is performed with 2 ul samples of this solution.

The apparatus used is a Packard Becker type 428 gas chromatograph with $F_{\text {. I }}$. detection; $180 \mathrm{~cm}$ glass column, internal diameter $0.2 \mathrm{~cm}$, packed with $3 \%$ OV -210 on Supelcoport $80 / 100$.

Temperatures: oven $250^{\circ} \mathrm{C}$, detector $270^{\circ} \mathrm{C}$, injection port $270^{\circ} \mathrm{C}$. Carrier gas nitrogen $2.1 \mathrm{~kg} / \mathrm{cm} 2(30$ p.s.i.). Attenuator position 32 , changed after 9 minutes to position 4 for internal standard. One gas chromatographic run takes about 15 minutes.

\section{c. Results:}

The overall recovery of added bile acids and internal standard is from $89.95 \%$ when ethyl acetate is used for extraction. The variation coefficient of the method is $5 \%$ for each substance. With derivatisation using HFIP it is possible to use peak heights instead of peak surfaces in the calculation, because with the OV-210 column there is a good peak symmetry [fig. 14.1), without considerable "tailing" in gas chromatography. 
Figure 14.1

Chromatogram of typlcal bile sample, containing lithocholic acid (peak 1), deoxycholic acid (peak 2), chenodeoxycholic acid (peak 3 ) and chollic acid (peak 4); internal standard 7-ketodesoxycholic acid (peak 5).

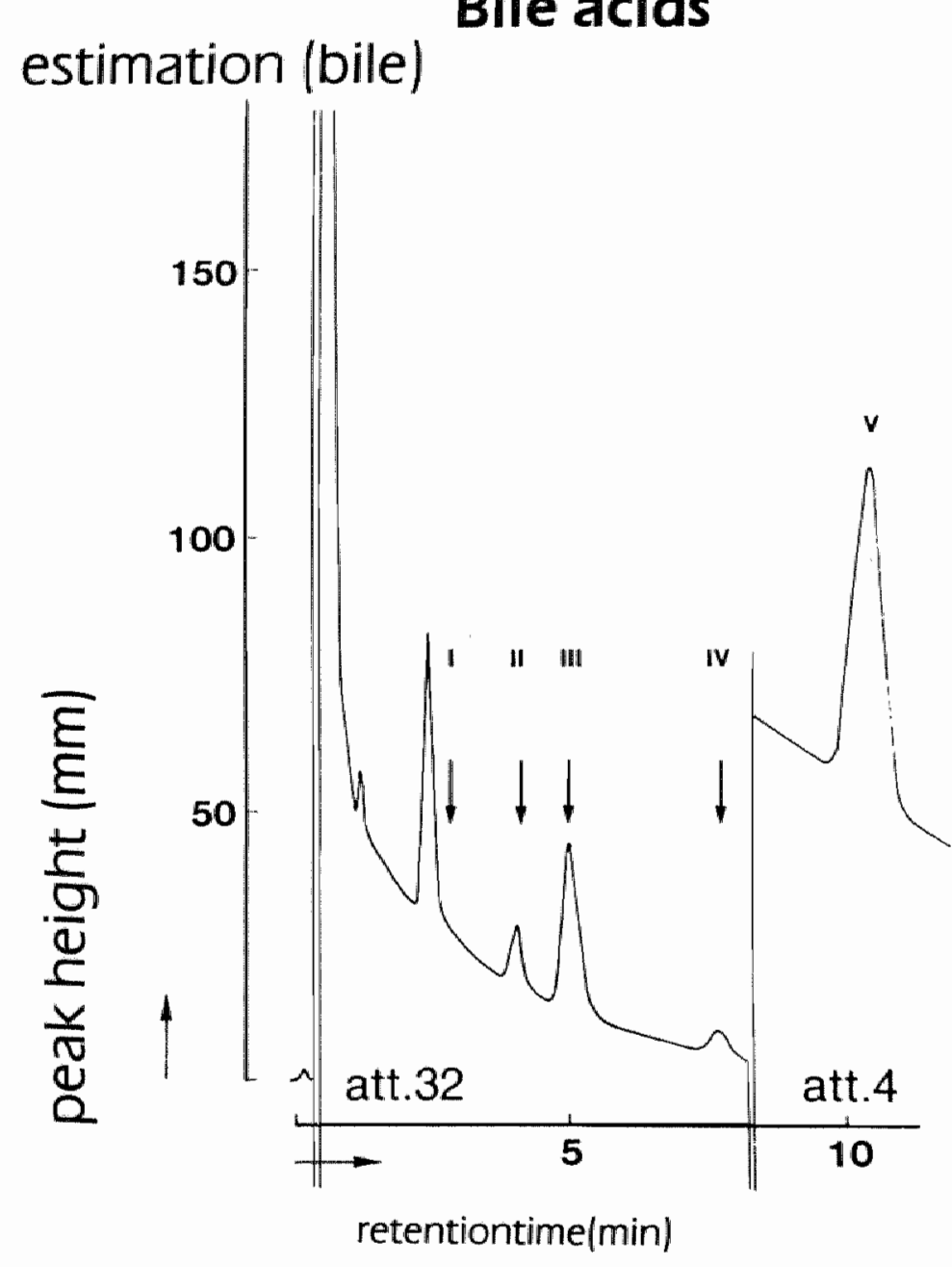

Automation of the method has been performed using the Packard Becker Autosampler model 605 with sample controller 103 and timer 104. Quantitation is performed by a Spectra Phycis Autolab system | using the internal standard method for the quantitation of the samples. For automatic liquid injection, the residues are dissolved in $1 \mathrm{ml}$ ethyl acetate and filled out in $2 \mathrm{ml}$ injection vials from which $2.5 \mathrm{ul}$ is injected at attenuator position 8. After every 10 samples a standard is analysed for recalibration. The run takes 15 minutes per sample. 


\section{d. Statistics:}

Because the results on BA, total as well as the individual BA's, do not represent stochastic variables, the interquartile range is preferred in arranging the laboratory data.

The results in peroperative bile samples between CDS patients and the control group are statistically compared using the test of Mann and Whitney itwo probes test of Wilcoxon]. The per- and postoperative data on bile composition in patients with CDS are revised using the symmetrical test of Wilcoxon.

\subsection{Results: Peroperative bile composition in CDS patients and control group}

In table 14.1 total BA in the peroperative bile samples are summarized in CDS

Table 14.1

Per- and postoperative data of total $\mathrm{BA}^{*}$ in hepatic bile in CDS patients and control group.

\begin{tabular}{|c|c|c|c|c|c|}
\hline \multicolumn{3}{|c|}{ CDS patients } & \multicolumn{3}{|c|}{ Control group } \\
\hline Patient & perop. data & postop.data & late postop. data & patient & perop. da \\
\hline 1. & 7.2 & 10.4 & 17.7 & 1. & 14.3 \\
\hline 2. & 3.1 & 7.0 & & 2. & 10.7 \\
\hline 3. & 5.2 & 7.6 & & 3. & 14.6 \\
\hline 4. & 2.6 & 4.2 & 7.1 & 4. & 11.4 \\
\hline 5. & 8.6 & 2.7 & & 5. & 24.7 \\
\hline 6. & 13.3 & 40.0 & & 6. & 20.2 \\
\hline 7. & 10.1 & 11.6 & & 7. & 13.7 \\
\hline 8. & 21.9 & 16.7 & 10.6 & 8. & 13.4 \\
\hline 9. & 26.9 & 8.7 & & 9. & 21.4 \\
\hline 10 & 49.0 & 40.3 & & 10. & 17.1 \\
\hline 11. & 37.0 & 45.7 & & 11. & 6.4 \\
\hline 12. & 1.9 & 6.7 & & 12 & 22.2 \\
\hline 13. & 38.0 & 44.7 & & 13. & 9.4 \\
\hline 14. & 13.5 & 29.6 & & 14. & 15.5 \\
\hline 15. & 10.4 & 52.6 & & 15. & 13.3 \\
\hline 16. & 21.5 & 14.8 & & 16 & 8.3 \\
\hline 17. & 12.3 & 10.5 & 12.2 & 17. & 13.1 \\
\hline 18. & 10.1 & 14.1 & & 18. & 120 \\
\hline 19. & 8.1 & 11.9 & 7.9 & 19. & 6.2 \\
\hline \multirow{3}{*}{ Median } & 10.1 & 11.9 & 10.6 & & 13.7 \\
\hline & \multirow{2}{*}{\multicolumn{3}{|c|}{ n.s. $(p=0.1)-$}} & & \\
\hline & & & & & \\
\hline
\end{tabular}


patients and the control group.

There was a wide variation in concentration in both per- and post- operative samples which was slightly greater in the controlgroup. Differences in bile viscosity are likely to be responsible for this phenomenon. This should have been corrected by taking the bile sample from the total daily bile production via the T-tube, at least in the postoperative samples.

No statistical difference could be established between the total BA concentration between peroperative samples from patients with and without $\operatorname{CDS}(p=0.1)$. The relative concentration of the four major $B A$ in patients with CDS are shown in table 14.2 and fig. 14.2. The comparable data on BA pattern from patients without CDS are summarized in table 14.3 and also in fig. 14.2. In the secondary BA of both patient groups only the medan value on DCA concentration was lower but the range in DCA concentration in CDS patients was too wide to be statistically significant. 


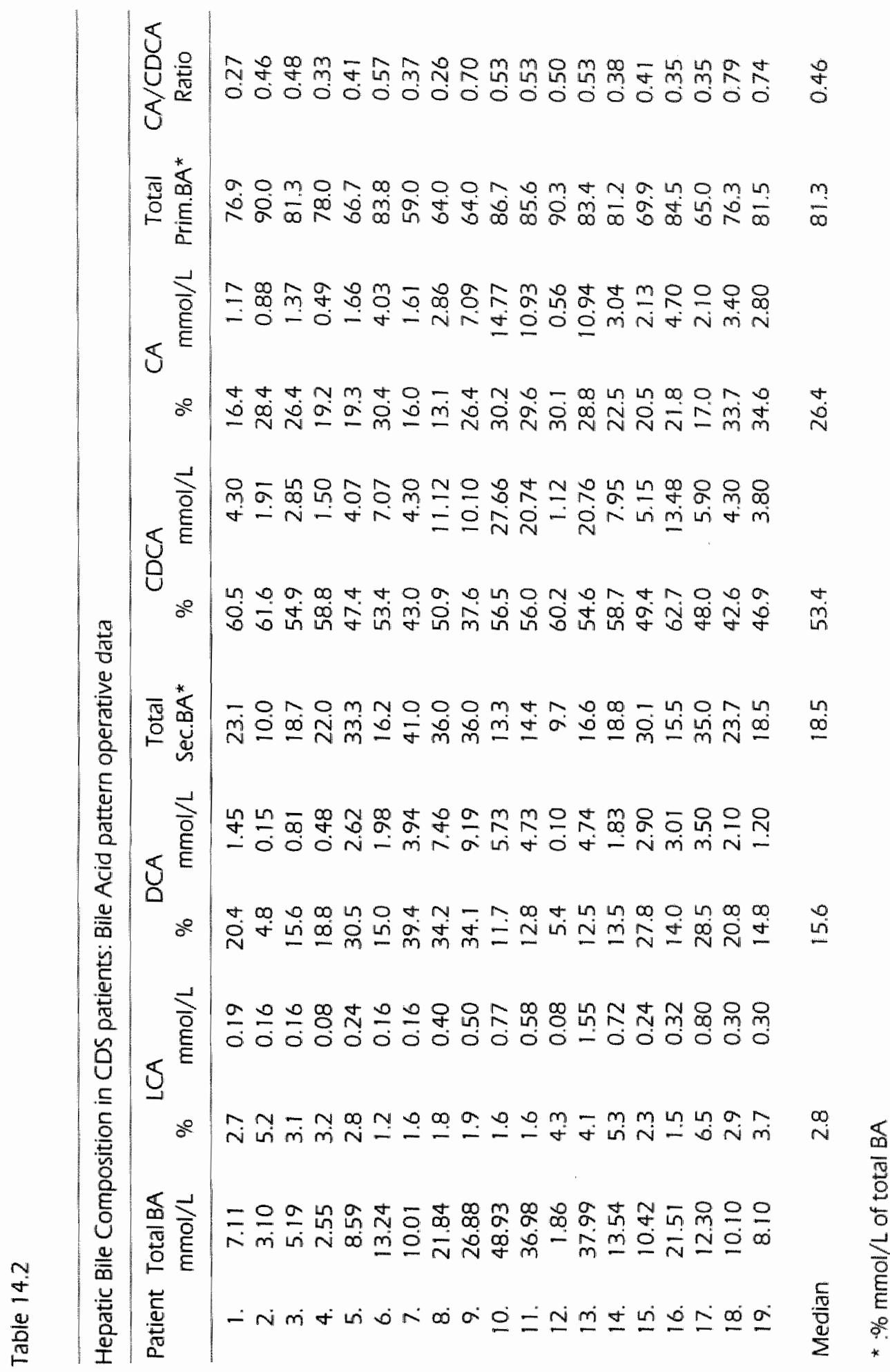




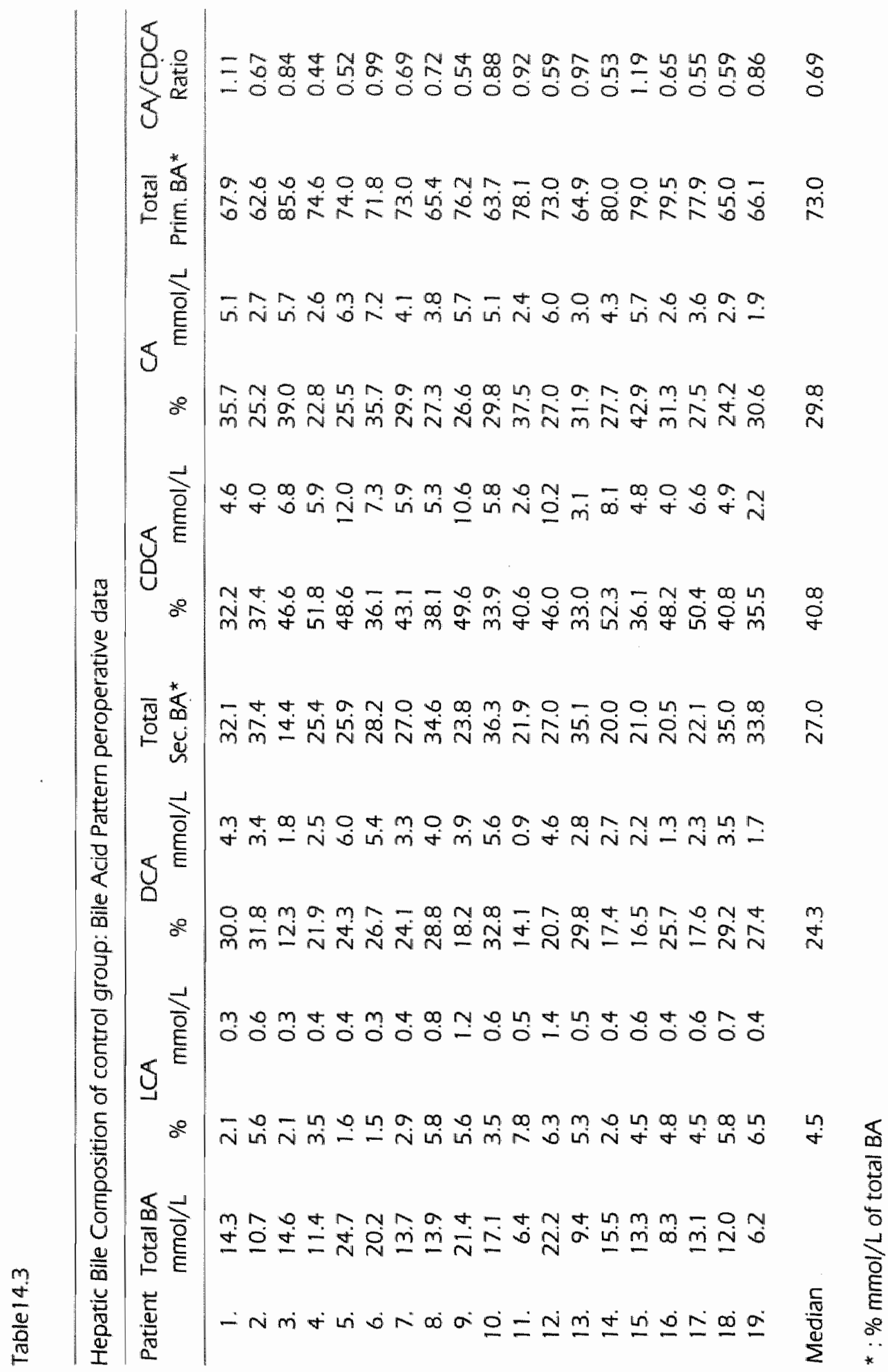


The total BA in CDS patients was only slightly increased but the corresponding total primary BA concentration did not show any statistical difference with the control group (fig. 14.3).

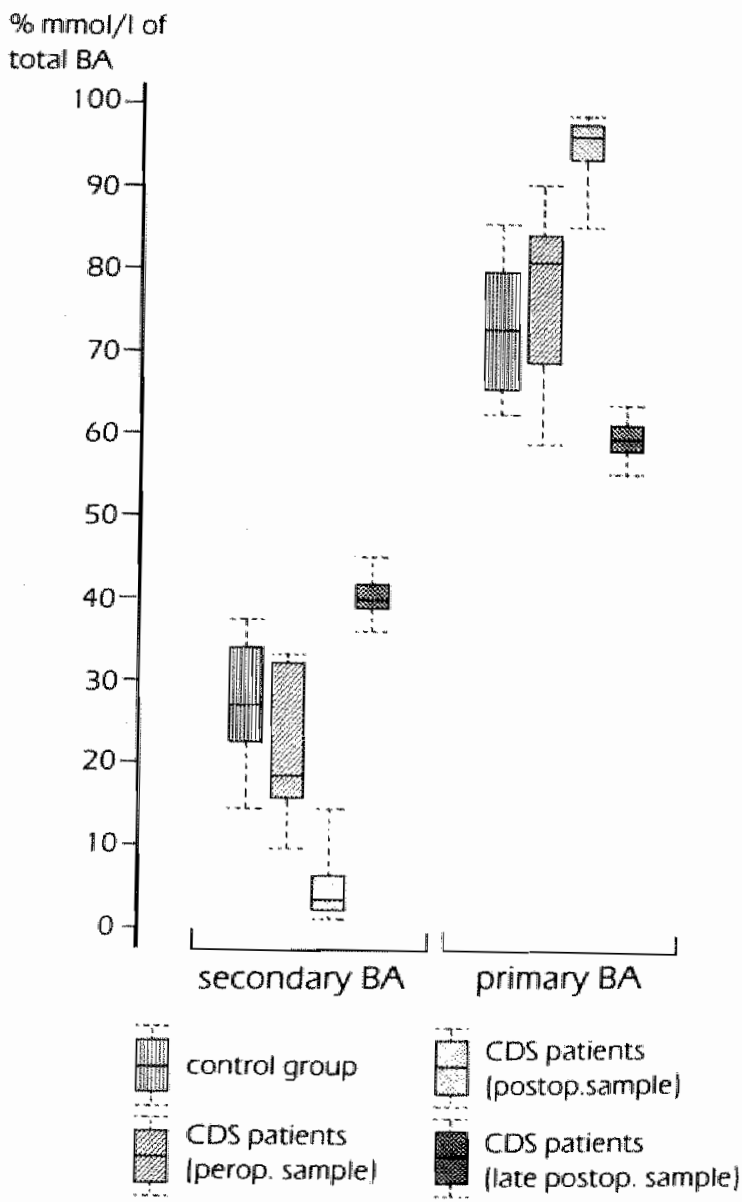

fig. 14.3

Because there was no difference in total $B A$ concentration in the peroperative bile samples of CDS patients and control group, the established differences in relative BA distribution are probably of minor importance as far as cholesterol solubility is concerned. The observed difference in CA and CDCA concentration between patients with and without CDS warrants further investigation especially the impact of bile flow disturbance on the synthesis of primary BA. This can be established with radioisotopes-labelled $\mathrm{BA}(30,102,172)$. 
Table 14.5

The per-and postoperative data of Cholestorol in CDS patients and control in hepatic bile

\begin{tabular}{|c|c|c|c|c|c|}
\hline \multicolumn{4}{|c|}{ CDSpatients } & \multicolumn{2}{|c|}{ contral group } \\
\hline & perop. data & postop. data & $\begin{array}{c}\text { late postop. } \\
\text { data }\end{array}$ & & perop data \\
\hline patient 1 & 1.6 & 4.0 & 0.8 & patient $1^{\circ}$ & 11.9 \\
\hline 2 & 0.4 & 1.5 & & $2^{\prime}$ & 3.6 \\
\hline 3 & 4.9 & 1.1 & & $3^{\prime}$ & 0.5 \\
\hline 4 & 4.3 & 0.5 & 0.3 & $4^{r}$ & 0.9 \\
\hline 5 & 3.6 & 0.5 & & $5^{\prime}$ & 0.1 \\
\hline 6 & 1.6 & 1.6 & & $6^{\circ}$ & 3.6 \\
\hline 7 & & 1.5 & & $7^{\prime}$ & 1.3 \\
\hline 8 & 2.7 & 2.0 & 0.4 & $8^{\prime}$ & 0.1 \\
\hline 9 & 0.3 & 0.9 & & $9^{\prime}$ & 1.8 \\
\hline 10 & 0.6 & 0.9 & & $10^{\prime \prime}$ & 0.1 \\
\hline 11 & 0.4 & 0.6 & & $11^{\circ}$ & 1.8 \\
\hline 12 & 2.9 & & & 12 & 0.3 \\
\hline 13 & 0.4 & 0.6 & & $13^{x}$ & 0.2 \\
\hline 14 & 1.6 & 1.6 & & $14^{\prime}$ & 0.2 \\
\hline 15 & 1.0 & 2.0 & & 15 & 1.1 \\
\hline 16 & 2.5 & 1.0 & & $16^{\prime \prime}$ & 0.8 \\
\hline 17 & 5.0 & 4.8 & 0.7 & $17^{\prime}$ & 0.1 \\
\hline 18 & 0.2 & 4.3 & & $18^{\prime \prime}$ & 0.5 \\
\hline 19 & 1.1 & 2.0 & 0.4 & $19^{\circ}$ & - \\
\hline \multirow[t]{2}{*}{ median } & 1.1 & 1.5 & 0.4 & & 0.9 \\
\hline & \multicolumn{3}{|c|}{ n.s. $|p=0.1|$} & & \\
\hline
\end{tabular}

Neither could there be established any statistical significant difference $(p=0.1)$ in cholesterol concentration in the peroperative bile in patients with and without CDS (table 14.5).

Postoperative bile lipid composition in CDS patients.

The results in BA composition are summarized in table 14.4 and fig. 14.2. The most striking difference in the postoperative phase is the very low percentage of any 


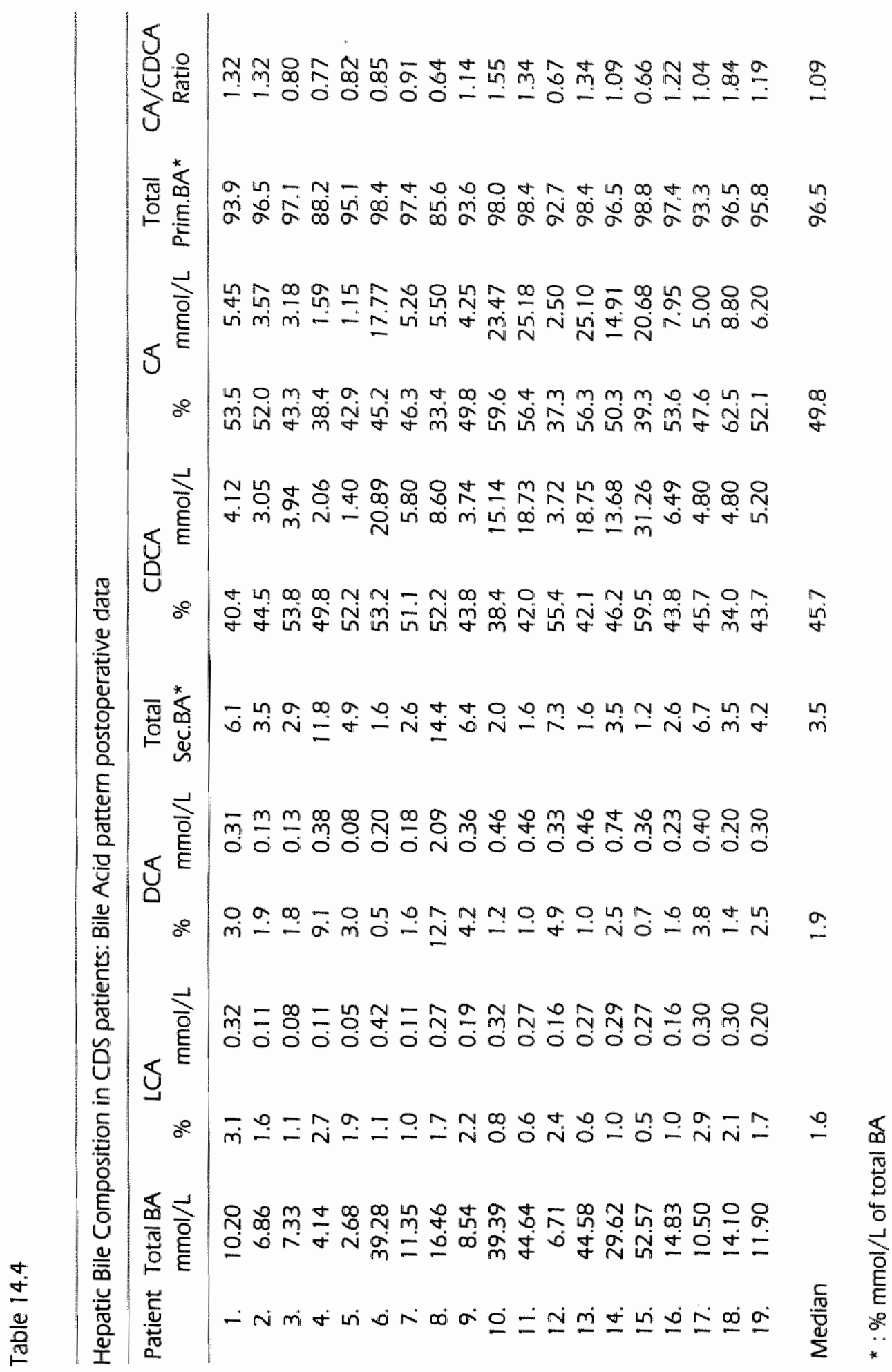


secondary BA, whether LCA or DCA. Almost all BA present in the postoperative sample are primary $B A: 96.5 \%$ of total $B A$, with an almost even distribution in $C A$ and $C D C A$ resulting in a CA/CDCA ratio being just over 1 (fig. 14.4). In 5 patients who underwent CDE with stone extraction, the BA pattern was

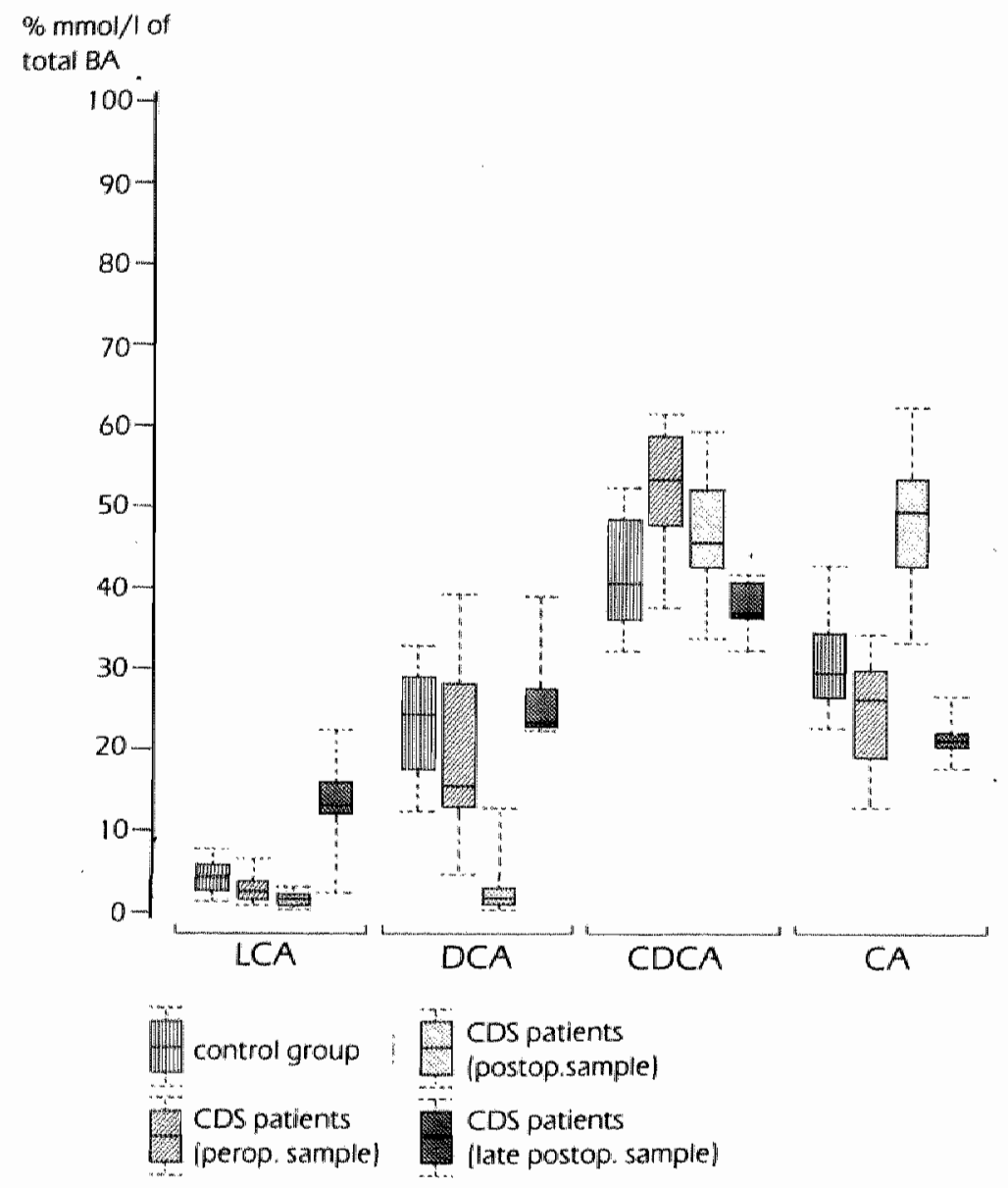

fig. 14.2 


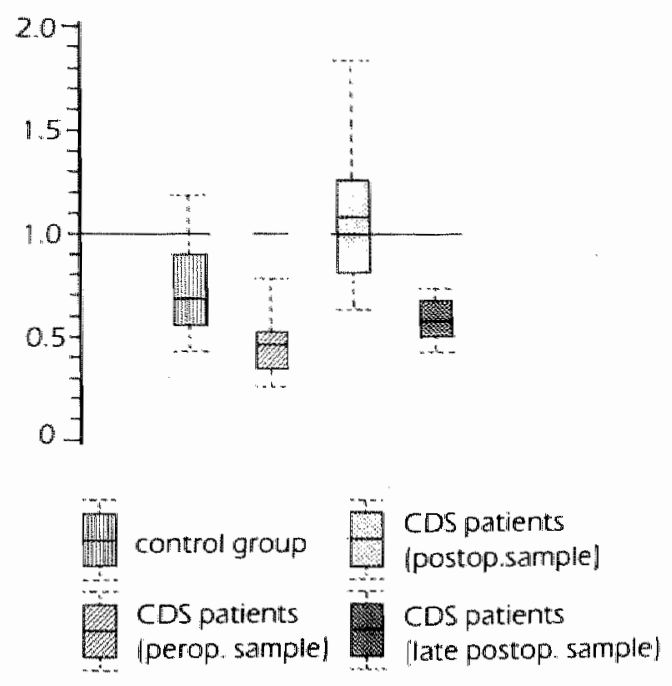

fig. 14.3

considerably changed 3 till 18 months postoperatively. In the late postoperative sample cholesterol concentration was significantly reduced $(p=0.05)$, although the number of patients investigated was rather small. Even the differences in median values between the postoperative and late postoperative cholesterol concentrations in CDS patients were statistically significant $(p=0.05$; table 14.5). 


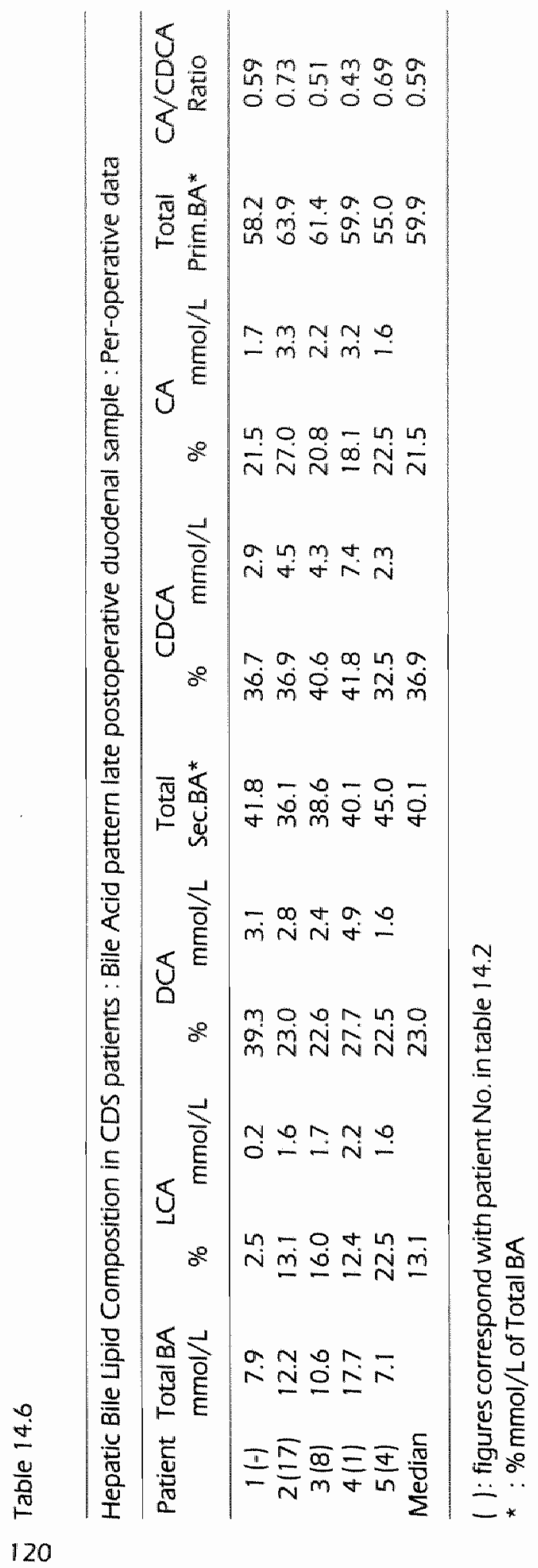


Total BA concentrations did not show any statistical difference in the per- post- and late postoperative samples (table 14.1). The relative distribution of the individual $B A$ however did notably change. The total primary BA was significantly reduced to $60 \%$ which was even less than the total primary BA in the control group peroperatively (fig. 14.3).

The percentage of CA did not significantly change from the peroperative CA concentration in CDS patients. The decrease in primary BA was mainly due to the CDCA fraction of total BA. The mean values of CDCA in the per-and late postoperative samples were 53.4 and $36.9 \%$ respectively. This difference almost equals the rise in LCA in, the late postoperative phase compared to the LCA concentration in the peroperative phase.

Consequently the total secondary BA concentration was raised considerably from $4 \%$ to almost $40 \%$ of total BA, mainly due to a rise in LCA.

\subsection{Discussion}

Hepatic bile lipid composition has been claimed to be the prime etiologic factor in stone formation $(288,291)$. Nevertheless, contrasting data in postoperative bile lipid composition have been published (Chapter 1).

In the patients investigated in this study there was no statistical difference in the main bile lipid components determining biliary cholesterol solubility, being total BA and cholesterol concentration. All samples were taken from patients after overnight: fasting.

Even in non-gallstone patients nocturnal lithogenic bile production is known to be $a$ normal physiological phenomenon. Using Indocyanide Green (ICG) to differentiate the partioning of nocturnally by secreted bile between gallbladder and duodenum, Van Berge Henegouwen could not demonstrate a preferential storage of lithogenic bile in the gallbladder during the night (49). Thus even the supersaturated nocturnal bile seems not to be an important pathogenic step in gallstone disease.

Northfield (259) could establish that a change in lithogenecity during the day was mainly due to a decrease in BA secretion. This diurnal variation has not been reported for cholesterol secretion, which is not influenced by meals (178).

The reported increase of lithogenic bile in the fasting state could not be approved in this study because there was no statistical difference in $B A$ concentration in the patients investigated.

However, the total BA concentration might thus be suspected to be higher during the day especially following stimulation by meals.

The influence of anesthesia on bile lipid composition, if present, was equal in CDS patients and in the control group in the peroperative samples. The postoperative and late postoperative samples were taken without anesthesia. The estimated difference in relative BA composition in the per-and postoperative samples are less likely to be induced by anesthesia because in both samples totally different changes on primary and secondary BA were noted. If any induction by narcotics is suspected 
this should have been on the hepatic phase in the EHC of BA. Although no prospective investigations on liver function tests have been done in the CDS patients, $B A$ synthesis may be suspected to be influenced by anesthetics resulting in a changing serum $B A$ composition secondary to differences in billiary BA. No extensive study of anesthesia and liver function especially concerning BA metabolism could be found in the literature. Anesthetic drugs altering bile secretion mediated by intrahepatic cholestasis have a direct effect on BA composition in bile and serum alike (152).

The most important and constant finding of anesthaesia induced hepatic derangement is impaired blood flow.

A deminished splanchnic blood flow by way of arterial hypotension, which is more pronounced with different anesthesiologic compounds, is a well established phenomenon. Also positive airway pressure impedes venous blood flow through the liver.

Currently, no major anesthesiologic effects on a pre-existent normal liver have been established. Only pre-existent liver disease and type of surgery performed are more important determinants of postoperative liver dysfunction than are anesthetic drugs (418).

Operative procedures on, but also near the liver, like cholecystectomy, are also associated with liver dysfunction postoperatively (420).

In conclusion, the influence of anesthesia on bile lipid composition in the per- and postoperative samples cannot be properly adjusted although on presently available data from the literature no major role can be ascribed to anesthesia being of serious consideration in the interpretation of the results on bile lipid composition in this study.

The differences in BA composition warrant further comment.

The main difference in BA composition in patients with and without CDS is a difference in primary BA. In patients with CDS the CDCA concentration was raised and $C A$ concentration was decreased. As has been outlined before, the design of this study does not permit interpretation on the precise mechanism. Without serum levels of both CA and CDCA estimated synchronically with biliary BA concentration, no accurate estimation can be made as to what extent $B A$ synthesis can be responsible for this change in relative $B A$ concentration.

In biliary obstruction, synthesis of CA is favoured over CDCA resulting in a high serum CA level as well as biliary CA concentration in the immediate postobstruction period [120]. CDCA might as well be transformed to CA in case of biliary obstruction as a detoxifying procedure because the three hydroxylated BA are supposed to be less toxic to the liver.

This has not yet been established in human physiology.

During bile outflow obstruction total BA production was established to be decreased favouring cholesterol stone formation (292). 
None of the patients with CDS included in this study was operated on during severe biliary outflow obstruction, which is illustrated by the level of DCA concentration found in the bile.

Why this seems to be a preferential pathway of synthesis of primary BA, namely CDCA in CDS patients, remains unresolved.

In the late postoperative bile the CA/CDCA ratio almost equals the ratio of the control group in the peroperative samples. So a high CDCA concentration seems to be a typical phenomenon in CDS patients but warrants further investigation (fig. 14.4).

Daily bile drainage sometimes almost equals bile production in the normal steady state. The T-tube acts like an external fistula in the EHC of BA. Synthesis of BA can thus become maximum. In animal experiments this method is widely used to estimate maximum BA synthesis because all BA circulating in the EHC are secreted. This has been convincingly reproduced in the bile samples taken from CDS patients in the postoperative period. A period of 24 hours clamping of the T-tube is apparently too short to reestablish the EHC of BA after external drainage. The late postoperative samples were taken 4 till 18 months postoperatively and all patients show a significant increase in secondary BA. To reestablish BA synthesis within EHC will take several weeks. However, during this period the cholesterol solubility will undoubtedly increase because the significant increase in primary BA will result in better micelle formation improving cholesterol solubility despite the total BA concentration did not improve. This is however only a temporary effect until the $E H C$ is stabilized. The high $C A / C D C A$ ratio will thus return to normal. Intestinal uptake of CDCA is almost $100 \%$ in jejunum and proximal ilium. A high $C A / C D C A$ ratio of intestinal contents reaching the caecum is thus suspected. This results in a rise in DCA because predominantly CA is converted to its secondary form by bacterial dehydroxylation in the colon.

A remarkable increase in LCA concentration is responsible for the rise in secondary $\mathrm{BA}$ in the late postoperative bille to almost $40 \%$. An increase in SBTT also for CDCA is thus suspected. In several studies only DCA concentration was raised in the postoperative bile and LCA concentration was not established, suspected to be of minor importance. Indeed DCA concentration did not show a difference from the control group. As has been stressed the difference in CDCA concentration in CDS patients in the per- and late postoperative sample equals the increase in LCA concentration in the late postoperative sample, suggesting an increase in SBTT particularly for CDCA. This rise in LCA concentration postoperatively is also quite different from the peroperative data in both control group and CDS patients. The rise in secondary $B A$ may be responsible for an increase in the frequency of diarrhea in patients following cholecystectomy. This is the main reason why vagotomy increases this risk when both procedures, cholecystectomy and truncal vagotomy. are performed simultaneously.

LCA is sulfated in the liver in the normal steady state. This decreases the toxicity of 
this secondary $\mathrm{BA}$, acting mainly on mucosa cells. However, the high increase in LCA as has been established in the late postoperative sample of operated CDS patients, should be reproduced to show any increased risk for carcinogenesis of the large bowel, despite the fact that the main proportion of LCA is thought to be sulfated (393).

The method of choice in BA estimation should be GLC - MS /Gas liquid Chromatography - Mass Spectometryl this time, being a more accurate laboratory method.

Nevertheless, Vernich (393, 393a) performed an epidemiological study and found an increased risk of right-sided colon cancer after cholecystectomy. There is no such correlation between cholelithiasis and colon cancer (218a). The increase in secondary BA following cholecystectomy especially may enhance carcinogenesis in the proximal large bowel where the newly formed $B A$, mainly DCA, are reabsorpted. Further evaluation is required to validate this hypothesis about the association of cholecystectomy and the increased risk for right-sided colon cancer. 


\section{In summary}

1. No statistical significant difference could be established in BA and cholesterol concentration in bile in gallstone patients with and without CDS.

2. Also the postoperative bile composition in CDS patients did not show any change in bile lipid composition favouring stone formation in the postoperative phase.

3. In the early postoperative bile a considerable increase in primary BA was established, favouring cholesterol solubility. This effect is suspected to be induced by T-tube drainage and is thought to be restored only after several weeks or months following $T$. tube extraction.

4. The relative BA composition did show a difference in CDCA concentration between CDS patients and the control group for which no explanation can be offered yet.

5. In the late postoperative bile a statistical significant decrease in cholesterol concentration was found. Total BA concentration did not change, resulting in a lower lithogenic bile in the late postoperative phase following cholecystectomy with CDE which is suspected to be permanent.

$6 . \quad$ In the late postoperative bile samples the most striking difference was a rise in LCA concentration, increasing total secondary BA fraction to almost $40 \%$.

The surgical implications of the above mentioned conclusions from this study are clear:

1. The hypothesis on reformed stones following cholecystectomy $1+/-\mathrm{CDE}]$ can be rejected and all biliary stones, even appearing years following the primary operation, should still be regarded as secondary (=retained) stones, unless otherwise proven.

2. The indication for bilio-intestinal bypass surgery should thus be reserved for "intractablle biliary stone disease" which is almost never encountered in cholesterol stone disease.

3. The increased concentration of secondary BA in the late postoperative phase lends credibillity to the hypothesis on the increased cancer risk in the postcholecystectomy patients. 


\section{Part IV}

Summary and conclusions References 


\section{Summary and conclusions}

In chapter 1 a review is presented on the etiology of cholelithiasis. Lithogenic bile production seems to be the primary etiologic factor. To what extend the gallbladder itself is involved etiologically is still uncertain. In the literature there is no concensus about the composition of bile following cholecystectomy. Because a lowered lithogenicity has been estasblished in postcholecystectomy patients, the gallbladder itself is suspected of having some influence on lithogenic bile production in the liver. Medical stone dissolution therapy has been started to reduce this primary event in the liver. The results are still disappointing because of a high percentage of recurrent stones.

The female prepondarance in cholelithiasis has not yet been convincingly explained by an essential difference in composition or synthesis of bile components. Oniy a larger Bile Acid Pool size has been estimated in female adults but is thought to be primarily related to gallbladder distention rather than a difference in synthesis. Lithogenic bile production in the liver as the main etiologic event in gallstone disease is thus seriously questioned. Local factors in the gallbladderwall may be more important etiologically than hitherto has been noticed.

The treatment of choice in gallstone disease still remains surgical therapy by way of cholecystectomy. The most important in biliary surgery remains the prevention of retained stones.

The etiology on CDS is still debated. The hypothesis of primary cholesterol stone formation within the biliary system is strengthened by an unknown percentage of retained stones following cholecystectomy.

The aim of this clinical study has been twofold: by standardizing $C D E$, including cholangioscopy, the overall retained stone incidence should be reduced.

Also hepatic bile has been investigated, in search for lithogenic bile in the postoperative phase. Reformed stones can thus be explained.

In chapter 2 different methods for establishing lithogenicity are presented. An extensive review is given of cholesterol and bile acid metabolism. The entero-hepatic circulation is discussed with special emphasis on the enteric phase of the individual $B A$. In the post-cholecystectomy state the total BA pool is redistributed resulting in a longer SBTT with a change in the relative composition of primary and secondary BA. In animal experiments bile obstruction has been shown to reduce $B A$ synthesis. A decrease in secretion of $B A$ results in a high lithogenic bile. This mechanism can be suspected of operating in case of biliary stones with a concomitant reduction in bile outflow. The influence of vagal stimulation and different hormones on bile lipid composition is discussed.

In chapter 3 some metabolic and endocrinologic disease entities which are related in some way to cholesterol stone disease, are debated. Special attention is paid to the influence ofoestrogen on bile secretion which results in some kind of cholestasis. The influence of progresterone is limited to a reduction in galbladder contraction. 
In chapter 4 several diseases are discussed which are suspected to be in some way related to cholesterol stone disease. Both acalculous cholecystitis and cholesterolosis have no relation whatsoever to gallstone disease. The complications in biliary pancreatitis with special emphasis on immediate and delayed surgical therapy are presented. A possible correlation between gallbladder carcinoma and gallstones is debated.

A review of medical treatment of gallstones with primary $B A$ is presented in chapter 5. Patient selection and adjunctive measures are most criticall for the ultimate success of the dissolution therapy. The high recurrence rates after discontinuance advocate intermittent treatment once dissolution has been successful.

In chapter 6 the financial repercussions of complicated biliary surgery are stressed. Prevention of retained stones seems most helpfull reducing the health care budget.

The clinical aspects of CDS are shown in chapter 7. Because the percentage of unsuspected stones is relatively high, operative cholangiography should be accepted as a standard procedure in every cholecystectomy. In a seperate heading the pathogenesis and treatment of pigment stone disease are outlined and differentiated from cholesterol stone disease. Special attention is paid to the problem of primary CDS. Differentiation between retained and reformed stones is hindered by the clinical phenomenon of silent stones. In case of partial or near total bile flow obstruction primary cholesterol stone formation is accepted.

Wide variation in RS percentages $(1 \pm 25 \%)$ is presented in the literature. In different studies the inferior or subsitandard quality of operative cholangiography has been determined retrospectively to be the main factor responsible for retained stones. Different anomalles in the biliary system, acquired as well as congenital, which promote the formation of retained stones, are discussed.

In chapter 8 a brief description is given on biliary anatomy with special reference to endoscopic inspection. The neural regulation of the sphincter mechanism is discussed.

All items concerned with CDE are eveluated in chapter 9, especially operative manometry and cholangiography. Prophylactic antibiotic administration seems indicated in high risk patients to reduce septic complications.

Extensive attention is paid to instruments and intraductal instrumentation with special emphasis on dilatation of the papilla which is regarded a dangerous and superfluous manoeuvre if cholangioscopic inspection precedes blind instrumentation.

Advantages and disadvantages of T-tube drainage are considered. In selected patients primary closure is sometimes justified.

In chapter 10 cholangioscopy is described starting with a brief historical review. The 
apparatus with accessories is presented. Indication(s) and contra indication(s) are outlined with an accurate description on operative technique. A standardized CDE including cholangioscopy is presented which was used prospectively in the clinical study described in chapter $\$ 3$.

Chapter 11 deals with non-operative treatment of retained stones. Mono-octanoine seems the drug of choice to resolve these stones by irrigation via the T-tube. The Burhenne technique is discussed.

Endoscopic sphincterotomy is extensively discussed with regard to its use as a primary procedure in gallstone patients. By improving biliary surgery the need for endoscopic treatment will be reduced considerably.

Operative treatment of retained stones by way of re-exploration is not advocated in the early postoperative phase because of high morbidity and mortality rates.

In chapter 12 the proper indication for the different bypass procedures are discussed with special reference to the primary operation.

In chapter 13 the results of $5 \frac{1}{2}$ years of billiary surgery in the De Wever Hospital are presented. During the first $1^{1 / 2}$ year CDE was performed without cholangioscopy. Following the routine use of cholangioscopy in a prospective clinical study, the RS percentage decreased dramatically. Only incidentally, mainly by inexperience of the operator, a stone was left behind.

During the last $21 / 2$ year the RS\% was $1 \%$.

Operative cholangiography and manometry were evaluated retrospectively. Manometry did not show any additional information to cholangiography to warrant its continued use as an operative diagnostic aid. The quality of cholangiography can be increased as will be explained. Above all, the value of the rigid cholangioscope in CDE has been convincingly demonstrated by the reduction in RS.

In chapter 14 the results of bile lipid composition studies are presented in patients with and without CDS: there is no statistically significant difference in the concentration of cholesterol and BA. The continued risk of stone formation postoperatively is thus seriously questioned.

In the direct postoperative phase cholesterol solubility improves. This is thought to be induced mainly by the external drainage through the T-tube: $B A$ are diluted out and primary BA for nation becomes maximal.

However in the late postoperative phase a significant decrease in cholesterol concentration is noted, which again disproves the very existence of recurrent, reformed and / or primary CDS!

Because the existence of both recurrent (reformed) and retained stones has been clearly demonstrated to be very unlikely in this clinical study using separate methods, a bilio-intestinal anastomosis as a primary treatment in CDS patients is not indicated if cholangioscopy is used without reservation in every CDE. 
This technique with the rigid cholangioscope reduces operative trauma by blind instrumentation. Papillary function can be clearly visualized which replaces dangerous dilatation of the papilla.

Because endoscopic inspection following stone extraction is superior to the postexploration T-tube cholangiography, the need for $C B D$ draining is reduced thereby promoting primary suturing of the CBD.

Considering the results on CDE the following standardized biliary exploration is proposed:

1. Operative cholangiography [without manometry]

2. Stone(s) extraction (reduce blind instrumentationl)

3. Endoscopic control

4. Residual stone extraction (with the scope)

5. T-tube completion cholangiography. 


\section{Samenvatting en conclusies}

In het eerste hoofdstuk wordt een overzicht gegeven van de factoren die een rol spelen bij het ontstaan van galsteenziekte. De lithogene galproduktie in de lever lijkt de primaire oorzaak. De rol van de galblaas is nog steeds onduidelijk. In de literatuur is geen eenstemmigheid omtrent de samenstelling van de gal na cholecystectomie. Omdat de gal minder lithogeen is na cholecystectomie, wordt verondersteld dat de galblaas zelf van invloed is bij het ontstaan van lithogene gal in de lever. De medicamenteuze therapie anticipeert op deze hypothese door de galzuursynthese in de lever te beinvloeden.

De duidelijk geslachtgebonden voorkeur van de galsteenziekte wordt niet verklaard door een essentieel verschil in galsamenstelling en/of productie. Daarentegen is wel een grotere "galzuurpool" bij vrouwen vastgesteld. Dit heeft eerder te maken met een hormonaal bepaalde dysfunctie van de galblaas dan met een verschil in galsecretie en/of synthese. In de pathogenese van de galsteenziekte lijken lokale factoren in de galblaas een belangrijker rol te spelen dan tot nog toe werd veronderstell.

De voorkeursbehandeling van galstenen is ondanks de nieuwe medicamenteuze behandeling nog steeds de chirurgische therapie. De chirurgische behandeling, ook van een ogenschijnlijk ongecompliceerd galsteenlijden, moet altijd gericht zijn op het voorkomen van retained stones.

De etiologie van stenen is nog steeds onderwerp van discussie. De hypothese van primaire cholesterolsteenvorming in de galwegen wordt mede bepaald door een onbekend percentage achtergebleven stenen na cholecystectomie.

Het doel van dit klinische onderzoek is tweeledig: door de chirurgische behandeling van choledochusstenen te verrichten volgens een gestandaardiseerde exploratie. waaraan toegevoegd de cholangioscopie, wordt gepoogd het RS percentage te verminderen.

Tevens worden in de levergal de twee belangrijkste galcomponenten bepaald te weten galzuren en cholesteral. Onderzacht wordt of op grond van een persisterende lithogene gall na cholecystectomie de hypothese omtrent "reformed stones" aannemelijk gemaakt kan worden. De indicatie voor een bilio-digestieve anastomose als primaire behandeling naast de choledochusexploratie zal dan moeten worden herzien.

In hoofdstuk 2 wordt een overzicht gegeven van de verschillende methodieken ter bepaling van de lithogeniciteit. Daarna wordt uitvoerig ingegaan op het metabolisme van het cholesterol en de galzuren. Behoudens synthese en secretie van beide galcomponenten wordt uitvoerig ingegaan op de pathofysiologie van de afzonderlijke galzuren. De entero-hepatische kringloop van de galzuren, met het accent op de darmfase, wordt beschreven. Het veranderde metabolisme van de galzuren na cholecystectomie vindt waarschijnlijk bij een aantal patienten reeds preoperatief plaats zodat de galblaas niet meer actief is betrokken bij de enterohepatische kringloop. Deze kortschakeling heeft tot gevolg dat de galzuren langer 
in de darm blijven dan bij de aanwezigheid van een normaal functionerende galblaas. Dit komt tot uitdrukking in de relatieve samenstelling van primaire en secondaire galzuren.

In dierexperimenten is gebleken dat bij een choledochusobstructie de galzuursynthese verminderd. Indien reeds galstenen aanwezig zijn in het biliaire systeem kan bij een partiele of totale afvloedbelemmering steenvorming geinduceerd worden door een verminderde uitscheiding van de galzuren zodat de concentratie van vrij-cholesterol toeneemt. Dit mechanisme zou van toepassing kunnen zijn bij de vorming van de zogenaamde "earthy stones": stenen samengesteld uit een harde kern met een zachte mantel.

Tot slot wordt de galsecretie besproken en tevens de invloed van diverse hormonen. Eveneens wordt aandacht besteed aan de invloed van de vagus op de galsamenstelling.

In hoofdstuk 3 worden enkele metabole en endocrinologische ziekten behandeid die in relatie staan tot de galsteenziekte. Met name wordt aandacht besteed aan de invloed van de geslachtshormonen, met het accent op de oestrogenen. Op grond van literatuurgegevens wordt een relatie verondersteld tussen een veranderde galzuursecretie en oestrogenen. Deze "fysiologische cholestase" kan manifest worden tijdens perioden van hyperoestrogenaemie, zowel medicamenteus gedurende pilgebruik als hormonaal tijdens de zwangerschap. De invloed van het progesteron beperkt zich waarschijnlijk alleen tot een veranderde galblaasfunctie.

In hoofdstuk 4 worden enkele ziektenbeelden behandeld waarvan een relatie met de galsteenziekte wordt verondersteld. Zowel bij acalculeuze cholecystitis ais de cholesterolosis bleek bij nadere literatuurstudie geen verband te bestaan met de steenziekte.

Op grond van de beschikbare literatuur wordt nader ingegaan op de relatie galsteenziekte en pancreatitis. Voor-en nadelen van directe en uitgestelde behandeling worden besproken.

In tegenstelling tot het cholangiocarcinoom wordt er een relatie verondersteld tussen galsteenziekte en galblaascarcinoom. Bij nadere analyse van de beschikbare literatuur is er alles behalve een directe relatie. Het tegendeel lijkt eerder waar.

In hoofdstuk 5 wordt er een overzicht gegeven van de medicamenteuze behandeling van galstenen met primaire galzuren. Naast indicatiestelling en ondersteunende maatregelen tijdens de behandeling worden de voor-en nadelen van de diverse primaire galzuren nader beschourd. Het succes van de behandeling wordt nog steeds voornamelijk bepaald door een strikte selectie van patienten. Het percentage recidief galstenen is relatief hoog zodat na het beeindigen van de therapie een remitterende behandeling ad infinitum lijkt te zijn aangewezen.

In hoofdstuk 6 wordt nader ingegaan op de financiële repercussies van de complicaties in de galwegchirurgie met accent op de behandeling van de patienten met achtergebleven stenen. Preventie van "retained stones" lijkt een wezenlijke 
bijdrage te leveren in de beheersing in de kosten van de gezondheidszorg.

Hoofdstuk 7 geeft een overzicht van de klinische aspecten van choledochusstenen. Er wordt met nadruk gewezen op de onvoorspelbaarheid van biliaire stenen en daardoor op de noodzaak van het routinematig verrichten van operatieve cholangiografie. In een apart hoofdstuk wordt de pathogenese en behandeling van pigmentstenen behandeld.

Uitvoerig wordt aandacht besteed aan de primaire choledochusstenen. De differentiatie ten opzichte van retained stones wordt bemoeilijkt door het fenomeen van "silent stones". Alleen afwijkingen in het galwegsysteem die aanleiding geven tot partiële dan wel totale afvoerbelemmering geven aanleiding tot het ontstaan van primaire cholesterolgalstenen.

In de literatuur wordt een variatie van retained stones percentage gevonden wisselend van 1 tot ruim 25\%. In diverse studies wordt de matige kwaliteit van de operatieve cholangiografie retrospectief aangeduid als zijnde een belangrijke factor in het ontstaan van de retained stones. Tevens worden enkele afwijkingen in het biliaire systeem aangegeven waardoor gemakkelijk stenen aan de aandacht ontsnappen tijdens de chirurgische exploratie.

In hoofdstuk 8 wordt een korte beschrijving gegeven van de anatomie van het galwegsysteem. De neuroregulatie van het sphinctermechanisme wordt nader beschouwd. Achtereenvolgens worden diverse afwijkingen beschreven in de galwegen voorzover deze kunnen worden herkent met de choledochoscoop.

In hoofdstuk 9 worden alle facetten van de choledochusexploratie waaronder drukmeting en cholangiografie op grond van literatuurgegevens geëvalueerd. Het al of niet toedienen van antibiotica als profylaxe tijdens galweg chirurgie lijkt gewettigd in risiko patienten.

Vervolgens wordt uitvoerig aandacht besteed aan instrumenten en instrumentatie. Met nadruk wordt gewezen op het bougigeren van de papil hetgeen als een kunstfout mag worden beschouwd zeker indien cholangioscopische inspectie daaraan voorafgaat. De voor- en nadelen van T-buisdrainage na choledochotomie worden geëvalueerd. In geselecteerde patienten kan primaire sluiting van de choledochotomie worden overwogen.

Hoofdstuk 10 behandelt de cholangioscopie. Na een kort historisch overzicht wordt een uitvoerige beschrijving gegeven van de cholangioscoop met hulpmiddelen. Indicatie(s) en contra-indicatie(s) worden duidelijk uiteengezet. Daarna volgt een nauwkeurige beschrijving van de techniek. Het standaardcholedochusexploratie protocal wordt beschreven met daarin opgenomen de cholangioscopie. Bovendien wordt de nadruk gelegd op het systematisch gebruik van de scoop tijdens elke choledochusexploratie.

Hoofdstuk 11 geeft een overzicht van de diverse behandelingsmethoden bij 
patienten waarbij onverhoopt een "retained stone" wordt gediagnostiseerd op het postoperatieve T-buis cholangiogram. De chemische behandeling van de reststenen door middel van Mono-octanoinespoelingen via de $\mathrm{T}$-buis lijkt een veelbelovende en efficiënte behandeling zodat instrumentaties via de T-buis fistel nog slechts sporadisch nodig zullen zijn.

Uitvoerig wordt aandacht besteed aan de primaire behandeling van choledochusstenen middels endoscopische retrograde sphincterotomie. Door het verbeteren van de chirurgische resultaten in de primaire behandeling, lijkt deze endoscopische methodiek als primaire behandeling minder aanwezig.

In hoofdstuk 12 wordt aandacht besteed aan de chirurgische behandeling van de "retained stones". Re-exploratie heeft een zeer hoge morbiditeit en mortaliteit. Recholedochotomie direct postoperatief moet daarom als een kunstfout worden beschouwd, zeker nu niet-operatieve technieken ter beschikking staan. In het tweede gedeelte van dit hoofdstuk worden de bypass operaties besproken. Omdat de (cholesterol)galsteenziekte op zichzelf een goede prognose heeft na een maximale chirurgische behandeling, te weten een standaard CDE inclusief cholangioscopie, behoort een bilio-digestieve anastomose als een overbodige ingreep te worden beschouwd. Het is een misvatting enige vorm van een biliodigestieve anastomose als preventieve maatregel te beschouwen voor een primair insuffiënte behandeling van choledochusstenen!

In hoofdstuk 13 worden de resultaten vermeld van $5 \frac{1}{2}$ jaar galwegchirurgie in het De Weverziekenhuis. Gedurende de eerste $1 \frac{1}{1} 2$ jaar werd geen cholangioscopie verricht. Behoudens standaardisering van de choledochusexploratie en invoering van de cholangioscoop werden geen andere maatregelen toegevoegd. Gedurende de laatste vier jaar is er een progressieve daling opgetreden in het retained stones percentage. Alleen in de beginperiode, door een geringe endoscopische ervaring van de diverse operateurs, werden incidenteel stenen achtergelaten. Zeer uitvoerig wordt ingegaan op de morbiditeit en mortaliteit gedurende de periode van onderzoek. Ook de indicatiestelling bij de diverse biliodigestieve anastomose operaties worden retrospectief nader bekeken. Apart worden beschouwd de operatieve cholangiografie en de manometrie. Deze laatste techniek lijkt weinig tot geen extra informatie toe te voegen aan de operatieve chalangiografie bij indicatiestelling tot choledochotomie.

Afgemeten aan het percentage vals positieve uitslagen kan de kwaliteit van de peroperatieve cholangiografie worden verbeterd. Daarentegen is de waarde van de peroperatieve cholangioscopie met de starre choledochoscoop onomstotelijk aangetoond. Met nadruk en bij herhaling wordt gewezen op de eenvoud van instrumentatie met deze choledochoscoop.

In hoofdstuk 14 worden de resultaten vermeld van de galsamenstelling bij patienten met en zonder choledochusstenen. Zowel de concentratie van cholesterol als totale gaizuren was niet significant verschillend tussen deze 2 groepen patienten, zodat 
galsteenvorming buiten de galblaas in het biliaire systeem minder waarschijnlijk wordt geacht.

In de direct postoperatieve fase treedt er een duidelijke verbetering op in galsamenstelling die de cholesteroloplosbaarheid bevordert. Dit wordt voornamelijk bewerkstelligt door een stijging van de galzuurconcentratie. Dit is waarschijnlijk slechts een tijdelijk effect veroorzaakt door de uitwendige drainage middels de Tbuis. Deze werkt als een soort fistel op de enterohepatische kringloop waardoor de galzuursynthese maximaal wordt. In de laat postoperatieve fase is een significante daling van het cholesterol percentage aantoonbaar. In de onderzochte patienten is het dus hoogst onwaarschijnlijk dat steenvorming alsnog plaats zal vinden door een definitieve vermindering in de gal lithogeniciteit.

Omdat hiermede de hypothese van reformed stones kan worden verworpen en mede door het invoeren van de cholangioscoop de kans op retained stones tot een uiterst minimum kan worden beperkt, is de indicatie voor het verrichten van een biliodigestive anastomose bij patienten met choledochostenen vooralsnog niet aanwezig. De chirurgische behandeling kan zich dus beperken tot een optimale choledochusexploratie waarbij cholangioscopie onontbeerlijk is.

Door de gemakkelijke hanteerbaarheid en het hoogwaardige optiek is de starre choledochoscoop een onmisbaar instrument geworden zodat een retained stone tot een hoogst curieuze complicatie kan worden gereduceerd.

Bovendien kan met behulp van de choledochoscoop het in den blinde instrumenteren worden beperkt. De papil kan optimaal worden geïnspecteerd in plaats van te sonderen. In deze studie is aangetoond dat de cholangioscopie een laatste controle middel is na steenextractie. Het verrichten van een per- en postoperatief $\mathrm{T}$-buis cholangiogram is dus minder dwingend geindiceerd zodat het primair sluiten van de choledochus in sommige patienten verantwoord wordt. 


\section{REFERENCES}

1 Accantino $L_{*, *} \operatorname{simon}$ F.R:

Identification and characterization of a bille acid receptor in isolated liver surface membranes.

J. Clin. Invest. $57: 496501,1976$.

2 Ackert J., Tobias $H_{\text {.: }}$ Primary sclerosing cholangitis.

Am. J. Gastroenterology 68: 498-500, 1977.

3 Acosta J.M. Rossi R. Galli O.M.R., Pellegrine C.A.:

Early surgery for acute gallstone pancreatitis : evaluation of a systematic approach.

Surgery $83: 367-370,1978$.

4 Adler R.D., Bennion L.J., Duane W.C., Grundy S.M. :

Effects of low dose chenodeoxycholic acid feeding on billiary lipid metabolism. Gastroenterology $68: 326-334,1975$.

5 Admirand W.H., Small D.M. : The physlochemical basis of cholesterol gallstone formation in man.

J. Clin. Invest. 47: 1043-1052, 1968.

6 Adson M.A.:

Carcinoma of the gallbladder.

Surg. Cl. North Am 53: 1201-1216, 1973.

7 Ahlberg ل.., Angelin B., Björkhem 1., Einarsson K.

Individual bile acids in protal venous and systemic blood serum of fasting man. Gastroenterology 73: 1377-1382, 1977.

8 Ahlberg J., Angelin B., Björkhem I., Einarsson K.:

Hepatic cholesterol metabolism in normo and hyperlipidemic patients with cholesterol gallstones.

J. Lip. Res. 20: 107-115, 1979.

9 Akiyama $H_{\text {: }}$

Unexpected problems of External Choledochoduodenostomy. Fiber scopic Examination in 15 patients.

Am. J. Surg. 140:660-665, 1980.

10 Alawneh I.:

Acute non-calculous cholecystitis in burns.

Br. J. Surg. 65: 243-245, 1978.

11 Allen B.L., Deveney CI.W., Way L.W.:

Chemical dissolution of bile ducts stones.

World J. Surg. 2: 429-437, 1978.

12 Allison J.G.

Cholecystocele. A congenital anomaly of the gallbladder.

Arch. Surg:: 113: 994-997, 1978.

13. Anderson M.C., Hauman R.L., Suriyaoa C., Schiller W.R.:

Pancreatic enzyme levels in bile of patients with extrahepatic biliary tract disease.

Am. J. Surg. 137:301-306, 1979. 
14 Ashur H., Siegal B., Oland Y, Adam Y.:

Calcified gallbladder (porcelain gallbladder).

Arch. Surg. 113: 594-596, 1978

15 Aubrey D.A., Edwards J.L.:

The selective use of combined supraduodenal and transduodenal exploration of the common bile duct.

Br. J. Surg. 65: 246-251, 1978.

16 Austin Jones S.:

Sphincteroplasty (Not Sphincterotomy).

Surg. Cl. North Am. 535: 1123-1137, 1973.

17 Austin Jones S.:

The prevention and treatment of recurrent bile duct stones by transduodenal sphincteroplasty.

World J. Surg. 2: 473-485, 1978.

18 Austin G.L., Johnson S.M., Shires G.T., Scott Jones R.:

The effect of feeding on the bile salt-independent canalicular secretion in dogs. Arn. J. Surg. 135: 36-39, 1978.

19 Axon A.T.R., Ashton M.G., Lintott D.J.:

Pancreatogram changes in patients with calculous biliary disease.

Br. J. Surg. 66: 466-470, 1979.

20 Back P.:

Differences in renal excretion between glyco-tauro, sulfo- and glucuronoconjugates of bile acids in cholestasis.

Falk Symposion 24, Basle 1976.

21 Baker P.R., Cuschieri A.:

Bile enzyme activities in patients with T-tube drainage following cholecystectomy.

Br. J. Exp. Path. 59: 277-281. 1978.

22 Bakes J.:

Die Choledochopapilloskopie, nebst Bemerkungen über Hepaticus: Drainage und Dilatation der Papille.

Arch. KI. Chir. 126:473, 1923.

23 Baldwin J., Heer W., Albo R.. Peloso O.

Effect of vagus nerve stimulation on hepatic secretion of bile in human subjects. Am. J. Surg. 111: 66-69, 1966.

24 Barbara L:

The medical treatment of cholesterol gallstones: experiences with

chenodeoxycholic acid.

Dig. 14:209-219, 1976.

25 Bartlett M.K:

Retained and Recurrent Common Duct Stones.

Am. Surg. 38: 63-68, 1972. 
26 Bartlett M.K. Waddell W.R.:

Indications for common duct exploration. Evaluation in 1000 cases.

N. Eng. J. Med. 258: 164-167, 1958.

27 Bateson M.C., MacLean D., Ross P.E., Bouchier I.A.D.:

Clofibrate therapy and gallstone induction.

Dig. Dis. 23: 623-628, 1978:

28 Been J.M., Bills P.M., Levis D.:

Electron probe microanalysis in the study of gallstones.

Gut 18: 836-842, 1977.

29 Bergmann F., Bandomer $G$., Herget H.J.:

The influence of $\beta$-sitosterol on biliary cholesterol saturation and bile acid kinetics in man.

Scan. J. Gastroenterol. 13:57-63, 1978.

30 Beher W.T:

Bile Acids. Chemistry and physiology of bile acids and their influence on atherosclerosis.

Karger S., Basel/New York, 1976.

31 Beinfield M., Hayes R.L.:

Use of intraoperative gram stain during cholecystectomy.

Am. J. Surg. 137: 773-774, 1979.

32 Bell G.D. Doran J.:

Gallstone dissolution in man using an essential oil preparation. Br. Med. J. 6155 :

$58,1979$.

33 Bell G.D., Mok H.Y. Thwe M.:

Liver structure and function in cholelithiasis.

Gut 15: 165-172, 1974.

34 Bennett P.H., Burch T.A., Miller M.:

Diabetes mellitus in American (Pimal Indians.

Lancet II: 125-128, 1971.

35 Bennion L. J., Ginsberg R.L., Garnick M.B., Bennett P.H.:

Effects of oral contraceptives on the gallbladder bile of normal women.

N.E.J. Med. 294: 189-192, 1976.

36 Bennion L.J., Grundy S.M.:

Effects of diabetes mellitus on cholesterol metabolism in man.

N.Eng. J. Med. 296: 1365-1371, 1977.

37 Bennion L.J., Grundy 5.M:

Risk factors for the development of cholelithiasis in man.

N. Eng. J. Med. 299:1161-1168, 1978.

38 Bennion L.J., Grundy S.M.:

Risk factors for the development of cholelithiasis in man.

N. Eng. J. Med. 299: 1221-1228, 1978. 
39 Bennion L.J., Matt D.M., Howard B.V:

Oral contraceptives raise the cholesterol saturation of bille by increasing biliary cholesterol secretion.

Metabolism 29: 18-22, 1980.

40 Benson E.A.:

Stone in bile duct with negative cholangiopancreatography.

Lancet $11: 1292,1977$.

41 Berci G., Hamlin J.A.:

Operative biliary radiology.

Williams \& Wilkins, Baltimore 1981.

42 Berci G., Johnson N.:

Functional studies of the extrahepatic biliary system in the dog by use of a controlled biliary fistula.

Ann. Surg. 161: 286-292, 1965.

43 Berci G., Majevska A., Smuckler E.A., Stevenson J.K.:

Der Effekt künstlicher Gallensteine beim Hund.

Langenibecks Arch. 308: 757-759, 1964.

44 Berci G., Shore J.M.:

Advances in cholangioscopy.

Endoscopy 4: 29-31, 1972.

45 Berci G., Shore J.M.:

Improved cannula for operative (cystic duct) cholangioscopy.

Am. J. Surg. 137: 826-828, 1979.

46 Berci G., Shore J., Hamlin J., Morgenstern L.

Operative fluoroscopy and cholangiography. The use of modern radiologic technics during surgery.

Am. J. Surg. 135: 32-35, 1978.

47 Bergdahl L., Holmlund E.W.:

Retained Bile Duct Stones.

Acta Chir. Scand. 142: 145-149, 1976.

48 Berge Henegouwen G.P. W.:

Lithogenic bile in functional disorders.

Proceedings Symposion on functional and acalculous anomalies of the gallbladder.

Exerpta Medica, Amsterdam, 1979.

49 Berge Henegouwen G.P. W., Hofmann A.F.:

Nocturnal gallbladder storage and emptying in gallstone patients and healthy subjects.

Gastroenterology 75:879-885, 1978.

50 Berk R.N., Armbuster T.G., Saltzstein 5.I.:

Carcinoma of the porcelain gallbladder.

Radiology 106: 29-32, 1973 
51 Best R.R., Rasmussen J.A., Wilson C.E:

An evaluation of solutions for fragmentation and dissolution of gallstones and their effect on liver and ductal tissue.

Ann Surg. 138:570, 1953.

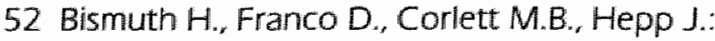

Long term results of Roux-en-Y Hepaticojejunostomy.

Surg. Gyn. Obst. 146:161-167, 1978.

53 Bismuth $H_{\text {, }}$ Kuntziger $H_{\text {, }}$, Corlette M.B.:

Cholangitis with acute renal failure: priorities in therapeutics. Ann. Surg. 181 : $881-887,1975$.

54 Black K., Hanna S.S., Langer B., Jirsch D.W.

Management of carcinoma of the extrahepatic bile ducts.

Can. J. Surg. 21:542-545, 1978.

55 Blackstone M.O., Nemchausky B.:

Chollangiographic abnormalities in ulcertative colitis assaciated pericholangitis which resemble sclerosing cholangitis.

Dig. Dis. 23: 579-585,1978.

56 Blumgart L.H., Carachi R., Imrie C.W., Benjamin I.S., Duncan J.G.:

Diagnosis and manangement of post-cholecystectomy symptoms: the place of endoscopy and retrograde choledochopancreatography.

Br.J.Surg. 64: 809-816, 1977.

57 Boer H.H.M. de:

Drukmeting gecombineerd met cholangiografie tijdens galblaas-en

galwegoperaties.

Ziekten v.d. galwegen, Symposium/Lunteren, 1974.

58 Bolck F., Machnik G.:

Zur Ursache, Form und Chemie der Gallensteine.

Naturwissenschaften 66:35-43, 1979.

59 Bordley IV J., Olsen J.E.:

The use of glucagon in operative cholanglography.

Surg. Gyn. Obst. 149: 583-584, 1979.

60 Bordley IV.J., Taylor White T.:

Causes for 340 reoperations on the extrahepatic bile ducts.

Ann.Surg. 189: 442-446, 1979.

61 Boston Collaborative Drug Surveillance Programme:

Oral Contraceptives and venous thromboembolic disease, surgically confirmed gallbladder disease, and breast tumours.

Lancet 1: 1399-1404, 1973.

62 Boston Collaborative Drug Surveillance Programme:

Surgically confirmed gallbladder disease, venous thromboembolism, and breast tumors in relation to postmenopausal estrogen therapy.

N. Eng.J.Med. 290: 15-18, 1974. 
63 Bouchier I.A.D:

The aetiology of gallstones.

Lancet I: 340-344, 1968.

64 Bouchier I.A.D:

The vagus, the bile, and gallstones.

Gut II: 799-803,1970.

65 Boyden E.A:

The Sphincter of Oddi in man and certain representative mammals.

Surgery 1:25-37, 1937.

66 Boyer J.L.:

New concepts of the mechanisms of hypatocyte bile formation.

Phys.Rev. 60:303-326, 1980

67 Braasch J.W.:

Carcinoma of the bile duct.

Am. Cl. North Am. 53: 1217-1227, 1973.

68 Braasch J.W., Roberts Fender H., Bonneval M.H.:

Refractory Primary Common Duct Stone Disease.

Am. J. Surg. 139: 536-530, 1980.

69 Brocks $H$ :

Choledochoscopy versus cholangiography. Experience of a 12-month trial.

Acta Chir. Scand. 118:434-438, 1959.

70 Brühl W.:

Der Einfluss der Cholezystektomie auf die Lipidzusammensetzung der Lebergalle. Nach Untersuchungen an 10 cholezystektomierten Patienten mit Rezidivisteinen in den Gallenwegen.

Schweiz. med. W/schr. 105: 426-496, 1975.

71 Brünner $H_{\text {, }}$ Rothmund $M$ :

Primärer Hyperparathyreoidismus, Pankreatitis und Cholelithiasis.

Dtsch. med. Wschr. 98: 425-429, 1973.

72 Burhenne H.J.:

Complications of nonoperative extraction of retained common duct stones.

Am. J. Surg. 131: 260-263, 1976.

73 Burhenne H.J.:

Nonoperative instrument extraction of retained bile duct stones.

World J. Surg. 2: 439-445, 1978.

74 Caldwell F.T.jr., Leveitsky K.:

Role of the gallbladder in the formation of gallstones.

Surg. Forum 17:353-355, 1966.

75 Caletti G.C.:

Endoscopic Retrograde Cholangiography (ERC) through Artificial Endoscopic

Choledocho-Duodenal Fistula.

Endoscopy 10: 203-206, 1978.

76 Cameron J.L., Gayler B.W., Harrington D.P.:

Modification of the Longmire procedure.

Ann. Surg. 187: 379-382, 1978. 
77 Carey M.C., small D.M:

The physical chemistry of cholesterol solubility in bile: relationship to gallstone formation and dissolution in man.

J. Clin. Invest. 61:998-1026,1978.

78 Carlsen J.E., Lauritzen T., Juul K. Hermann Ch.:

Common duct stones in patients with acute cholecytitis.

Acta Chir. Scand. 143:47-48, 1977.

79 Carruthers $\$ . G$., Dujovne C.A.

Digoxin therapy during T-tube biliary drainage in man. JAMA 240: 2756-2757, 1979.

80 Caspary W.F.:

Increased deconjugation of Bile Acid and vitamin B12 malabsorption in Diabetics and treatment with Bigunides. In: Bile Acid Metabolism in Health and Disease. Falk Symposion 24, MTP Lancaster, England, 1977.

81 Chainoff Ch., Menache R:

Bile composition and osmolarity in the interpretation of formation of bile stones and their classification.

Br. J. Surg. 66: 476-477, 1979.

82 Champeau M., Pineau P.. Léger P.:

Chirurgie du foie et des voies biliaires.

Ed. Méd. Flommarion, Paris 1966.

83 Chaude S. Devitt J.E.:

T-tubes, the surgical amulet after choledochotomy.

Surg. Gyn. Obst. 136: 100-102, 1973.

84 Cheung L.Y., Chang F.C.:

Intravenous cholangiography in the diagnosis of acute cholecystitis.

Arch. 5urg. 133: 568-570, 1978.

85 Cheung L.Y., Englert E., Moody F.G.

Dissolution of gallstones with bile salts, lecithin, and heparin.

Surgery 76:500-503, 1974.

86 Chriantiansen L.Y., Nielsen OV., Efsen $F_{\text {: }}$ :

Non-operative treatment of retained bile duct calculi in patients with an indwelling T-tube.

Br. J. Surg. 65: 581-584, 1978.

87 Cole W.H., Harridge W.H.:

Disappearance of "stone" shadows in postoperative cholangiograms.

JAMA 156: 238-243, 1957.

88 Collins P.G., Redwood C.R.M., Wynne-Jones G.

Common Bile-Duct suture without intraductal drainage following choledochotomy.

Br. J. Surg. 47: 661-667, 1960

89 Collins P.G.:

Further experience with common bile-duct suture without intraductal drainage following choledochotomy.

Br. J. Surg. 54:854-856, 1967. 
90 Conley D.R., Coyne M.J., Chung A.:

Mechanism of bile acid diarrhea: the role of cyclic AMP in colonic secretion.

Am.J.Clin.Dis. 21:453-458, 1976.

91 Corlette M.B., Schatzki S., Ackroyd F.:

Operative cholangiography and overlooked stones.

Arch. Surg. 113: 729-734, 1978.

92 The coronary drug project research group.

Gallibladder disease as a side effect of drugs influencing lipid metabolism.

N. Eng. J. Med. 296: 1185-1 190,1977.

93 Cotton P.B.:

Non-operative removal of bile duct stones by duodenoscopic sphincterotomy.

Br. J. Surg. 67: 1-5, 1980.

94 Cowen A.E., Korman M.G., Hofmann A.F.:

Metabolism of lithocholate in healthy man.

Gastroenterology 69: 67-76, 1975.

95 Cowie A.G., Sutor D.J.:

Viscosity and osmolarity of abnormal bile.

Dig. 13: 312-315, 1975.

96 Cox J.L., Helfrich L.R., Pass H.I., Osterhaut S.:

The relationship between biliary tract infections and postoperative

complications.

Surg. Gyn. Obst. 146: 233-236, 1976.

97 Coyne M.J., Marks J., Schoenfield L.J.:

Mechanism of cholesterol gallstone formation.

Cl. Gastroenterology 6: 129-139, 1977.

98 Csendes A.:

Pressure measurement in the biliary and pancreatic duct systems in controls and in patients with gallstones, previous cholecystectomy or Common duct stones.

Gastroenterology 77: 1203-1210, 1979.

99 Csendes A., Larach J., Godoy M.:

Incidence of gallstones development after selective hepatic vagotomy.

Acta Chir. Scand. 144: 289-291, 1981.

100 Daly J.M., Bowen J.:

Multiple intrahepatic and extrahepatic biliary cysts.

South. Med. J. 171:604-606, 1978 .

101 Daniel O., Singh M.L:

Clinical value of measurement of flow and pressure in the common bile-duct.

Ir. J. Med. Sci. 480: 415 e.v., 1965.

102 Danzinger R.G., Hofmann A.F., Thistle J., Schoenfield L.J.:

Effect of oral chenodeoxycholic acid on bile acid kinetics and billary lipid composition in women with cholelithiasis.

J.Cl.Invest. 52: 2809-2821, 1973. 
103 Dardik $H_{\text {, }}$ Beneventano T., Rosen R., Gliedman M.L.:

Experimental management by nonoperative adrenergic stimulation of simulated common duct stones.

Am. J. Dig. Dis. 16:321-326, 1971.

104 Dardik H., Gliedman M.L. Christ R, schein Cl.J.:

Neuroendocrine influence on the dynamics of the choledochal sphincter.

Surg. Gyn. Obst. 131:675-678, 1970.

105 Dardik H., Schein Cl.J., Warren A., Gliedman M.L:

Adrenergic receptors on the canine biliary tract.

Surg. Gyn. Obst. 128:823-826, 1969

106 De Bernardis F., Tosto S., Silvestre G., Binda $R_{\text {.: }}$

Ascaris infestation of biliary tract.

Acta Chir. Ital. 33: 125-135, 1977.

107 Dekking $F$ :

Van de koele meren des doods. Pseudomonas in het ziekenhuis.

N.T.V.G. 1 10: 1637-1640, 1966

108 Delikaris P.G., Machail P.O., Klonis G.D., Haritopoulos N.C.:

Biliary bacteriology based on intraoperative bile cultures.

Am. J. Gastroent. 68: 51-55, 1977.

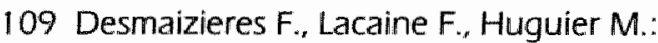

La cholangiographie per-opératoire: valeur diagnostique dans la lithiase biliare.

Nouv. Press Méd. 7: 1455-1457, 1978.

110 Detrie Ph., Barthelemy P., Kiniffo H.V.:

Syndrome du canal cystique.

Presse Med. 47: 2075-2076, 1970.

111 Devlin H.B., Sahay A.K., Tiwari P.N., Galvin P.B.:

Cholecystectomy and a simple technique of operative cholangiography.

Br. J. Surg. 65:848-851, 1978.

112 Domellöf $L$., Rydh $A$., Truedson $H_{\text {.: }}$ :

Leakage from T-tube tracts as determined by contrast radiolophy.

Br. J. Surg. 64:862-863, 1977.

113 Dowling R.H.:

Chenodeoxycholic acid therapy of galistones.

Cl. Gastroent. 6: 141-163, 1977.

114 Duane W.C.:

Simulation of the defect of bile acid metabolism associated with cholesterol cholelithiasis by sorbitol ingestion in man.

J. Lab. Clin. Med. 91: 969-978, 1978.

115 Duane W.C. Hanson K.:

Role of gallbladder emptying and small bowel transit in regulation of bile acid pool size in man.

J. Lab. Clin. med. 92:858-872, 1978.

116 Du Priest R.W., Khaneja S.C., Adams Cowley R.T.:

Acute cholecystitis complicating trauma.

Ann. Surg. 189: 84-89, 1979 . 
117 Dye M., MacDonald A., smith G.:

The bacterial flora of biliary tract and liver in man.

Br. J. Surg. 65: 285-287, 1978.

118 Deyhle P:

Endoskopische Papillotomie: eine echte Alternative zur Chirurgie?

Schweiz. Med. W/schr. 108:845-846, 1978.

119 Doran J.r Bell G.D., Fayadh M.:

Effect of ioglycamide (Biligram) on bile flow and biliary lipid secretion in man.

Gut 19: 300-307, 1978 .

120 Ekdahl P.H. Sjovall J.:

On the conjugation and formation of bile acids in the human liver.

Acta Chir. Scand. 114:439-452, 1957.

121 Elfstrom J.:

The timing of cholecystectomy in patients with gallstone pancreatitis.

Acta Chir. Scand. 144:487-490, 1978.

122 Ellis G., Goldberg D.M., Spooner R.J., Milford Ward A.:

Serum enzyme tests in diseases of the liver and biliary tree.

Am.J. Cl. Path. 70:248-258, 1978.

123 Engin A., Haberal M., Sanac Y.:

Side-to-side choledochoduodenostomy in the management of choledocholithiasis.

Br. J. Surg. 65: 99-110, 1978.

124 Exalto J.:

Ulcus jejuni na gastro-enterostomie.

N.T.V.G. 55: 469, 1911.

125 Faber R.G., Ibrahim S.Z., Thomas D.M., Beynon G.P.J., et al.:

Gallstone disease presenting as septicaemic shock.

Br. J. Surg. 65: 101-105, 1978.

126 Finegold S.M.:

Anaerobes in biliary tract infection.

Arch. Int. Med. 139: 1338-1339, 1979.

127 Finnis D., Rowntree T.:

Choledochoscopy in exploration of the common bile duct.

Br. J. Surg. 64:661-664, 1978.

128 Fitzpatrick G., Neutra R., Gilbert J.P.:

Cost-effectiveness of cholecystectomy for silent Gallstones.

In: Costs, Risks, and Benefits of Surgery; P. 246-261 Ed.: Bunker, J.P.

Oxford University Press, N.Y. 1977.

129 Fleury A., Poxon V., Dorricott N., Keighley M.:

Influence of cholecystokinin on pyloric reflux after operation for gallstones and peptic ulcer.

Proc. Roy. Soc. Med. 70: 367-368, 1977.

130 Fogarty T., Krippaehne W.W.W., Dennid D.L., Fletcher W.S.:

Evaluation of an improved operation technic in common duct surgery.

Am. J. Surg. 116:177-183, 1968. 
131 Freund $H_{\text {. }}$ Charuzi I. Grantit G., Berlatzky $Y$ :

Choledochoduodenostomy in the treatment of benign biliary tract disease.

Arch. Surg. 112: 1032-1034, 1977.

132 Fromm $H$ :

Studies of liver function and structure in patients with gallstones before and during treatment with chenodeoxycholic acid.

Acta Hepatogastroenterol. 22: 359-369, 1975.

133 Fromm H., Hofmann A.F.:

Breath test for altered bile acid metabolism.

Lancet 11: 621-622, 1971.

134 Garcia-Romero E, Lopez-Cantarero M., Arcelus M:

Dissolution of human gallstone with clofibrate.

J. Surg. Res. 24:62-64, 1978.

135 Gardner B., Dennis Ch., Patti J., Chenouda M.:

The effect of para-aminobenzoic acid and taurocholic acid feeding on humanbile composition.

Surg. Gyn. Obst. 144:879-882, 1977.

136 Gilliland S.E., Speck M.L.:

Deconjugation of bile acids by intestinal lactobacilli.

Appl. Env. Microbiol. 33: 15-18, 1977.

137 Glassman J.A.

Tricks, tactics and techniques for the removall of impacted gallstones from the common bile duct.

Surg. Gyn. Obst. 151: 99-106, 1980.

138 Glenn F.:

Atlas of biliary tract surgery.

Appleton, Century Croft, New York 1963.

139 Glenn F:

Retained calculi within the biliary ductal system.

Ann. Surg. 179: 528-539, 1974.

140 Glenn $F$.:

Trends in surgical treatment of calculous disease of the biliary tract.

Surg. Gyn. Obst. 140:877-884, 1975.

141 Glenn F::

Acute acalculous cholecystitis.

Ann. Surg. 189:458-465, 1979.

142 Glenn F., Beil A.R.:

Choledocholithiasis demonstrated at 586 operations.

Surg. Gyn. Obst.: 499-506, 1964.

143 Glenn F. Mcsherry C.K.

Gallstones and pregnancy among 300 young women treated by

cholecystectomy.

Surg. Gyn. Obst. 127: 1067-1072, 1968. 
144 Glenn F., McSherry C.K., Dineen P.:

Morbidity of surgical treatment for nonmalignant billiary tract disease.

Surgery 136:15-26, 1968 .

145 Goldberg P.B., Long W.B., Oleaga J.A. Mackie J.A.

Choledochocele as a cause of recurrent pancreatitis.

Gastroenterology 78: 1041-1045, 1980.

146 Goldberg M.J., Smith J.W., Nichols R.E:

Comparison of the fecal microflora of Seventh-Day Adventists with individuals consuming a general diet. Implications concerning colonic carcinoma.

Ann.Surg. 186: 97-100, 1977.

147 Goldstein F., Thornton J.J., Szydlowski T.:

Biliary tract dysfunction in giardiasis.

Dig. Dis. 23: 559-560, 1978.

148 Goodhart G.L., Levinson M.E., Trotman B.V., Soloway R.D.:

Pigment vs cholesterol cholelithiasis. Bacteriology of gallbladder stone, bile, and tissue correlated with biliary lipid analysis.

Dig. Dis. 23: 877-882, 1978.

149 Grace D.M., Henderson A.R.:

Preoperative and postoperative enzyme changes in patients with gallstones.

Surg. Gyn. Obst. 147:219-224, 1978.

150 Grauthoff $H_{\text {., }}$ Thelen $M$. Peters $M$ :

Vergleich der Wertigkeit von intravenosem bzw. Infusionscholangiogramm

sowie intraoperativem und Tomocholangiogramm.

Fortschr. Rontgenstr. 127: 575-577, 1977.

151 Greenwald R.A., Pereiras R., Morris S.J., Schiff E.R.:

Jaundice, choledocholithiasis and a nondilated common duct.

JAMA 240: 1983-1984, 1978:

152 Greim H., Trülzisch D., Czygan P., Rudick J.:

Mechanism of cholestestasis. Bile acids in human liwers with of without billiary

obstruction.

Gastroenterology 63: 846-850, 1973.

153 Greim H., Trülizsch D., Roboz J., Dressler K.

Mechanism of cholestasis. Bile acids in normal rat livers and in those after bile duct ligation.

Gastroenterology 63: 837-845, 1973 .

154 Griffin W.T.:

Choledochoscopy.

Am. J. Surg. 132:697-698, 1976.

155 Grosze H.:

Cancer risk in different types of gallstones.

Arch. Geschwülstforsch. 47: 710-718, 1977.

156 Gruder E:

Width of the common bile duct in cases of cystic duct occlusion by an impacted

calculus.

Acta Rad. 3: $481-484,1965$. 
157 Gumico J.J., Valdivieso V.D.:

Studies on the mechanism of ethinyl estradiol impairment of bile flow and bile salt excretion in the rat.

Gastroenterology $61: 339-341,1971$.

158 Gunn A:

The use of tantalum clips during operative cholangiography.

Br. J. Surg. 67: 146, 1980 .

159 Haag W:

Gallemwegsanomalien. Uberblick und Bericht uber zwei distale

intrapankreatische Gallengänge.

Langenbecks Arch. Chir. 344:115-122, 1977.

160 Hanemaayer R.:

De "achtergelaten" galsteen.

N.T.V.G. 108: 494-495, 1964.

161 Harrison E.C., Roschke E.J., Meyers H.I., Edmiston W.A.:

Cholelithiasis: a frequent complication of artificial heart valve replacement.

Am. Heart J. 95: 483-488, 1978.

162 Hay A.M.:

A suction cannulator for rapid peroperative cholangiography.

Br. J. Surg. 66: 675, 1979.

163 Heater B., Sterry Ashby B.:

Choledochoscopy appearance of hepatic ducts in polycystic disease of the liver. J. Roy. Soc. Med. 71:526-529, 1978.

164 Hegardt F.G., Dam H.:

The solubility of cholesterol in aqueous solutions of bile salts and lecithins.

Z. Ernährungsw. 19:223, 1971.

165 Heil Th. ,Behohlavek D., Merkle N., Voss E.U.:

Die chirurgische Papillenplast endoskopisch-manometrische und Klinische Befunide.

Langenbecks Arch. Chir. 346: 59-64, 1978.

166 Heimbach D.M., White T.T.:

Immediate and long term effects of instrumental dilation of the sphincter of Oddi.

Surg. Gyn. Obst. 148: 79-80, 1979.

167 Heller A.M., Hohl R., Madhavan T., Wong K.:

Retropharyngeal abcess after endoscopic retrograde cholangiopancreatography:

an uncommon but potentially fatal complication.

South. Med. J. 171:219-221, 1978.

168 Helpap B.:

Malignant Papillomatosis of the intrahepatic bile ducts.

Acta Hepato-Gastroenterology 24: 419-425, 1977.

169 Henrion C., Wallon P., Eygen B.:

Cholangiographie intraveineuse pré-opératoire et calcul résiduel du cholédoque.

J. Chir. 115:577-584, 1978. 
170 Hepner G.W.. Hofmann A.F., Malagelade J.R., Szczepanik P.A.:

Increased bacterial degradation of bile acids in cholecystectomized patients.

Gastroenterology 66: 556-564, 1974.

171 Hepner G.W., Hofmann A.F., Thomas P.J.:

Metabolism of steroid and amino acid. Moieties of conjugated bile acids in man.

1. Cholylglycine.

J. Clin. invest. 51, 1889-1897, 1972

172 Hepner G.W., Hofmann A.F., Thomas P.J.:

Metabolism of steroid and amino acid. Moieties of conjujated bile acids in man.

II. Glycine-conjugated dihydroxy bile acids.

J. Clin. Invest. 51: 1898-1905, 1972.

173 Hicken N.F., MCAllister A.J.:

Operative cholangiography as an aid in reducing the incidence of

"overlooked"common bile duct stones: a study of 1.293

choledocholithotomies.

Surgery 55: 753-758, 1964.

174 Hilles T.M., W/estbrook K.C., Caldweil F.T.:

Surgical injury of the common bile duct.

Am. J. Surg. 134: 712-716, 1977.

175 Hoare A.M., McLeish A., Thompson H., Alexander-Willems J.:

Selection of patients for bile diversion surgery: use of bile acid measurement in fasting gastric aspirates.

Gut 19: 163-165, 1978.

176 Hofmann A.F.:

Desaturation of bile and cholesterol stone dissolution with chenodeoxycholic acid.

Am. J. Clin. Nutr. 30: 993-1000, 1977.

177 Holman J.M., Rikkers L.F., Moody F.G.:

Sepsis in the management of complicated billiary disorders.

Am. J. Surg. 138: 809-813, 1979.

178 Holzbach R.T., Marsch M., Olszewski M., Holan K.:

Cholesterol solubility in bile. Evidence that supersaturated bile is frequent in healthy man.

J. Cl. Invest. 52: 1467-1479, 1973.

179 Hopton D:

Common bile duct perfusion combined with operative cholangiography.

Br. $\Perp$. Surg. 65: 852-854, 1978.

180 Horntrich 1 ., Keuntje $H_{\text {.: }}$

Early operation in biliary surgery?

Zentralbl. Chir. 102: 1489-1500, 1977.

181 Howat H.T., Sarles H.:

The Exocrine Pancreas.

W.B. Saunders CO. LTD, London, 1979. 
182 Huang Chia-Ssu.:

Development of surgery in China in the last fifteen years.

Chin. Med. J. 92:1-10, 1979.

183 Hunt D.R., Blumgart L.H."

latrogenic choledochoduodenal fistula.

Br. J. Surg. 67: 10-13, 1980.

184 Huybregtse K.:

Endoscopische papillotomie en verwijdering van choledochusstenen.

Symposion Choledocholithiasis, N.V.G.E. 1979.

185 Iseli A., Marshall V.C.

Choledochoscopy: a comparison of a rigid and a flexible fibreoptic instrument. Med. I. Aust. 1: 131-132, 1978.

186 iser J.H., Dowling H., Mok H.Y. Bell G.D.:

Chenodeoxycholic acid treatment of gallstones. A follow-up study report and analysis of factors influencing response to therapy.

N. Eng. J. Med. 293:378-383, 1975

187 Jakimowicz J.J.:

Post-operatieve mogelijkheden met de flexibele choledochoscoop.

Workshop choledochoscopie, N.V.H., Rotterdam 1980.

188 Jakimowicz J.J., Stockmann C.H.J.:

Diffuse papillomatosis of the biliary tract.

Arch. Chir. Neerl. 29: 255-257, 1977.

189 Jarhult J., Falck B., Ingemansson S., Nobin A.:

The functional importance of sympathetic nerves to the liver and endocrine pancreas.

Ann. Surg. 189: 96-100, 1979.

190 Javitt N.B.:

Hepatic bile formation (Second of two parts).

N. Eng. J. Med. 295: 1511-1516, 1976.

191 Johnson A.G., Harding A.J.:

Prevention and treatment of recurrent bile duct stones by

choledochoduodenostomy.

World J. Surg. 2: 487-496, 1978.

192 Johnson L.F., Walta D.C.:

Intrahepatic pigment stones. An asian disease diagnosed with retrograde cholangiography.

Dig. Dis 23 (supl.): 135-185, 1978.

193 Jutras J.A., Longtin J.M., Llevesqu H.P.:

Hyperplastic cholecystoses.

Am.J. Roentgenol. 83:: 795-798, 1960.

194 Juvara I. Vereanu i.. Huch A.

Etude du relief endocholédocien.

J. Chir. 106, 599-608, 1973. 
195 Kaminski D.L., Barner H.B., Codd J.E., Wolfe B.M.:

Evaluation of the results of external choledochoduodenostomy for retained. recurrent, or primary common duct stones.

Am. J. Surg. 137: 162-166, 1979.

196 Kaminski D.L., Dorighi J., Jellinek M.:

Effect of electrical vagal stimulation on canine hepatic bile flow.

Am. J. Phys. 227: 487-493, 1974.

197 Kappas A., Alexander-Williams J., Keighley M.R.B., Watts G.T.: Operative choledochoscopy.

Br. J. Surg. 66:177-179,1979.

198 Karran S.:

Controversies in surgical sepsis.

Praeger 1980.

199 Kavlie $H_{.}$, W/hite T.T.:

Flow rates and manometry in the assessment of the common bile duct.

Acta Chir. Scand. 138:817-826, 1972.

200 Keeman J.N., Delfgaauw P.H.A.J., Bergman R., Greep J.M.:

Achtergebleven choledochusstenen.

"Ziekten van de galwegen": symposium Lunteren 1974.

201 Kinne M.J.:

Persistent cholecystohepatic ducts.

Arch. Surg. 155: 972-974, 1980.

202 Koch $H_{\text {., Stolte }}$., Walz $V$.:

Endoscopic lithotripsy in the common bile duct.

Endoscopy 9: 95-98, 1977.

203 Koch M.M., Freddara U., Lorenzini i., Giampieri A.:

A stereological and biochemical study of the human liver in uncomplicated cholelithiasis.

Digestion 18: 162-177, 1978 .

204 Kaehler I.S., Chistides P.S., Adamec T.A.:

A complication of percutaneous cholangiography resulting in hypoxia and death of an anaesthetized patient.

Anesthesiology 49: 210-212, 1978.

205 Kotiger Y.S.:

On pathogenesis of functional disturbances of the pancreas in patients with cholelithiasis.

Vestn. Khir. 104: 43, 1970.

206 Kroesen G., Bodner E., Russe W., Troyer E.:

Beëinflüssung der intraoperativen Cholangiometrie durch

Anaesthesiemethoden.

Anaesthesist 27:21-24, 1978 .

207 Kutz K., Miederer S.E., Paumgartner G.:

Chenodeoxycholic acid therapy of intrahepatic radiolucent gallstones in a patient with Caroli's syndrome.

Acta Hep. Gastroent. 25: 398-401, 1978. 
208 Kurtz L.W., Wise L.:

A study of the impact of resident participation on the results af surgery for cholecystitis.

Surgery $86: 530-535,1979$

209 Kyösola K:

Adrenergic and cholinergic innervation of the supraduodenal common bile duct.

Am. J. Gastroent. 70:179-183,1978.

210 Lahtinen J., Alhava E.M. Aukee S:

Acute cholecystitis treated by early and delayed surgery.

A controlled clinical trial.

Scand. J. Gastroent. 13:673-678, 1978.

211 Lam S.K., Tsui J.K.C., Chan P.K.W., Wong K.P.:

How often does bacteraemia occur following endoscopic retrograde

cholangiopancreatography (ERCP).

Endoscopy $4: 231-234,1977$.

212 Landsman J.N.:

Indicatie cholecystectomie, ter preventie van het galblaascarcinoom. Ziekten wan Galwegen, Lunteren 1974.

213 Lechin F., van der Dijs B., Bentolila A., Pesa F.:

Adrenergic influences on the gallbladder emptying.

Am. J. Gastroent. 69: 662-668, 1978.

214 Léger L., Liguory A., Soprani A., Kanoui F:

Vésicule biliaire intra-hépatique compliqué d"une lithiase cholédocienne postopératoire. Intéret de la sphinctérotomie endoscopique.

Nouv. Presse Méd. 7: 2049-2051, 1978.

215 Leissner K.H., Wedel H., Schersten T.:

Comparison between the use of oral contraceptives and the incidence of surgically confirmed gallstone disease.

Scand. J. Gastroent. 12:893-896, 1977.

216 Linden W. v.d. Sinzel $H_{\text {.: }}$

Ideal cholecystectomy: in an early stage at an early age.

Acta Scand. Chir. 142:62-66, 1976.

217 Linden W/ v.d. Bergman $F$.:

An analysis of data on human hepatic bile. Relationship between man in bile components, serum cholesterol and serum triglycerides.

Scand. J. Clin. Lab. Invest. 37: 741-747, 1977.

218 Lindgren B., Petersson B.G., Sorbris R., Bengmark S.:

Changes in the costs of elective cholecystectomies, 1955-1965-1975.

Ann. Surg. 189:447-454, 1979.

218 a Linos A.L. et al:

Cholecystectomy and carcinoma of the colon

Lancet If: $379-381,1981$ 
219 Lipton S., Caralps-Riera J., Estrin J.:

The plasma clot extraction of biliary duct calculi: a preliminary report.

Surgery 70: 746-750, 1971 .

220 Loeb P.M., Wheeler H.O., Berk R.N.:

Endoscopic pancreatocholangiography in the diagnosis of biliary tract disease.

Surg. Cl. North. Am. 53: 1007-1018, 1973.

221 Longmire W.P.:

When is cholangitis sclerosing?

Am. J. Surg. 135: 312-320, 1978.

222 Lou M.A., Mandal A.K. Alexander J.K., Thadepalli H.:

Bacteriology of the human biliary tract and the duodenum.

Arch. Surg. 112: 965-967, 1977.

223 Low-Beer T.S., Nutter S.:

Colonic bacterial activity, biliary cholesterol saturation and pathogenesis of gallstones.

Lancet II: 1063-1065, 1978.

224 Low-Beer T.S., W/icks A.B.C.r. Heaton K.W., Durrington P.:

Fluctuations of serum and bile lipid concentrations during the menstrual cycle.

Br. Med. J. 1: 1568-1570, 1977.

225 Lund J.:

Surgical indications in cholelithiasis: prophylactic cholecystectomy elucidated on the basis of long-term follow up on 526 non-operated cases.

Ann. Surg. 151: 153-162, 1960.

226 Lupinetti M., Mehigan D., Cameron J.L.:

Hepatobiliary complications of ulcerative colitis.

Am. J. Surg. 139: 113-118, 1980.

227 Lux G.:

Endoscopic papillotomy. The development of a method.

Endoscopy 10: 206-208, 1978.

228 Lundstrom B., Holm D.:

Bile Duct Diameter and Diagnostic Reliability at Cholangiography.

Acta Chir. Scand. 145: 105, 1979.

229 Lykkegaard Nielson M:

Gastrin and gastric acid secretion in hepaticojejunostomy Roux-en-Y.

Surg. Gyn. Obst. 150: 61-64, 1980.

230 Madden J.L.:

Primary common bile duct stones.

World J. Surg. 2: 465-471, 1978.

231 Madura J.A., Loomis R.C., Harris R.A., Grosfeld J.:

Relationship of obesity to bile lithogenicity in man.

Ann. Surg. 189: 106-111. 1979.

232 Makayama F., Linden W. v.d.:

Stratification of bile in gallbladder and gallstone formation.

Surg. Gyn. Obst. 141:587-590, 1975. 
233 Malleki M., Faghiti M., Golshan M.:

Primary sclerosing cholangitis.

South. Med. J. 71:855-858, 1978.

234 Marks J.W., Conley D.R., Capretted T.L., Bonorris G.G.:

Gallstone prevalence and biliary lipid composition in inflammatory bowel

disease.

Dig. Dis. 22: 1097-110, 1977.

235 Marks J.W. "Sherman G.M., Bornorris G.G., Chung A.:

Gallstone dissolution by chenodeoxycholic acid and phenobarbital.

Am.J. Gastroent. 69: 160-165, 1978.

236 Maton P.N., Murphy G.M., Dowling R.H.:

Ursodeoxycholic acid treatment of gallstones. Dose-response study and possible mechanism of action.

Lancet II: 1297-1301, 1977.

237 Matsumoto Y., Uchida K., Nakase A., Honjo J.:

Congenital cystic dilatation of the common bile duct as a cause of primary bile duct stone.

Am. J. Surg. 134: 346-352, 1977.

238 Maudgal D.P., Bird R., Blackwood W.S., Northfield T.C.:

Low-cholesterol diet: enchancement of effect of CDCA in patients with gallstones.

Br. Med. J. 2:851-853, 1978.

239 Mazzariello R.M.:

Review of 220 cases of residual biliary tract calculi treated without reoperation: an eight-year study.

Surgery 73: 299-306, 1973.

240 McCarthy J.D.:

Radiomanometric guides to common bile duct exploration.

Am. J. Surg. 34:697-701, 1977.

241 McDonald A.C., McKay C., McAllister R.:

The effect of insulin and atropine on bile volume and composition.

J. Surg. Res. 23: 155-160, 1977.

242 McDougall R.M., Walker K., Thurston O.G.:

Prolonged secretion of lithogenic bile after cholecystectomy.

Ann.Surg. 182: 150-153, 1975.

243 Mciver M.:

An instrument for visualizing the interior of the common duct at operation.

Preliminary note.

Surgery $9: 112-114,1941$.

244 McSherry C.K.:

Clinical approaches to calculous biliary tract disease: third annual Leon

Ginzburg lecture.

Mount Sinai J. Med. 45: 729-737, 1978. 
245 Mekhjian H.S. Philips S.F., Hofmann A.F.:

Colonic secretion of water and electrolytes induced by bille acids: Perfusion studies in man.

J. Clin. Invest. 50: 1569-1577, 1971.

246 Miederer S.E., Lindstaedt H., Siedek M., Franken Th.:

Endoskopische transpapillare Spaltung einer Choledochocele.

Dtsch. Med. Wschr. 103:216-219, 1978.

247 Millbourn E:

On Re-operation for Choledocholithiasis.

Acta Chir. Scand. 99:285-312, 1950.

248 Mirizzi P.L:

Operative Cholangiography.

Surg. Gyn. Obst. 65: 702, 1937.

249 Montet J.C., Gerolami A.:

Intrahepatic metabolism and secretion of biliary lipids.

Digestion 17: 346-364, 1978.

250 Morelli A., Narducci F., Ciccone R.:

Can Mirizzi Syndrome be classified into acute and chronic form? An endoscopic retrograde cholangiography (ERC) study.

Endoscopy 10: 109-112, 1978.

251 Morran C., McNaught W., McArdle C.S.:

Prophylactic co-trimoxazole in biliary surgery.

Br. Med. J. 2: 462-464, 1978.

252 Moulijn A.C:

Over de waarde van de röntgen-diagnostiek bij het galsteenlijien.

Proefschrift Nijmegen 1973.

253 Moulijn A.C.:

De per-operatieve en post-operatieve cholangiografie.

Ziekten van de Galwegen, Lunteren 1974.

254 Nakagawa S., Makino I., Ishizaki T., Dohi I.

Dissolution of cholesterol gallstones by ursodeoxycholic acid.

Lancet I: 376-369, 1977.

255 Newman H.F.:

Complications of cholelithiasis.

Am. I. Gastroenterology 50: 476-496, 1968.

256 Nielsen M.L., Justesen T, Lenz K:

Bacterial flora of the small intestine and bile acid metabolism in patients with hepatico-jejunostomy.

Scand. J. Gastroenterology 12:977-982, 1977.

257 Nix GA.J.J.:

Personal communication.

258 Nora P.F., Berci G., Dorazio R.A., Kirschenbaum G.:

Operative cholediochoscopy. Results of a prospective study in several institutions.

Am. J. Surg. 133: 105-110, 1977. 
259 Northfield T.C., La Russo N.F., Hofmann A.F. Thistle J.L.:

Biliary lipid output during the day and an overnight fast II. Effect of chenadeoxycholic acid treatment in gallstone subjects.

Gut 16:12-17, 1975.

260 Ohmori K., Kinoshita $H_{\text {. }}$ Shiraha $Y$., Satake $K$ :

Pancreatic duct obstruction by a benign polypoid adenoma of the ampulla of Vater.

Am. J. Surg. 132:622-623, 1976.

261 Okabe N., Kawai K., Kondo O. Machida T.

Operative and postoperative choledochofiberscopy.

Am. J. Surg. 137:816-820, 1979.

262 Ollinger P.:

Zur Frage der Nachbeschwerden nach Gallensteinoperationen.

Langenbechs Arch, u Dtsch. Z. Chir. 288: 6-16, 1958.

263 Opie E. in:

The theory of retrojection of bile into the pancreas.

Rev. Surg. 27: 1-7, 1963.

264 Orloff M.J.:

Retained and recurrent bile duct stones: introduction.

World J. Surg. 2: 40 i-402, 1978.

265 Orloff M.J.:

Importance of surgical technique in prevention of retained and recurrent bile duct stones.

World J. Surg. 2: 403-410, 1978.

266 Osnes M., Larsen 5., Lowe P., Gronseth K.:

Comparison of endoscopic retrograde and intravenous cholangiography in diagnosis of biliary calculi.

Lancet II: $230,1978$.

267 Otten $G_{\text {., Helwing }}$., Lux M:

Störung der Magenentleerung bei Gallensteinträgern.

Wien. Med. Wisch. 24:741-742, 1977.

268 Ottinger L.W., Warshaw A.L., Bartlett M.K.:

Intraoperative endoscopic evaluation of the bile ducts.

Am. J. Surg. 127:465-468, 1974.

269 Papachristodoulou A.J., Mackenzie A.r Norman J., Karran S.J.:

Single dose Cephazolin prophylaxis in biliary tract surgery.

J. Roy. Coll. Surg. Ed. 23: 178-183, 1978.

270 Patchen Dellinger E., Kirschenbaum G., Weinstein M., Steer M.:

Determinants of adverse reaction following postoperative T-tube cholanglogram.

Ann. Surg. 191:397, 1980.

271 Partington Ph.F.:

Twenty-three years of experience with sphincterotomy and sphincteroplasty for stenosis of the sphincter of Oddi.

Surgery 145:161-168, 1977. 
272 Pearlman B.J., Schoenfield L.J.:

Gallstones: present and future of medical dissolution.

Med. Cl. North Am. 62: 87-105, 1978.

273 Pennington C.R., Ross P.E., Bouchier I.A.D.:

Fasting and postprandial serum bile acid concentrations in normal persons using an improved GLC method.

Digestion 17:56-62, 1978

274 Petersen S.R., Sheldon G.F.:

Acute Acalculous Cholecystitis a complication of hyperalimentation.

Am. J. Surg. 138: 814-817, 1979.

275 Perpetuo M.O., Valdivieso M., Heilbrun L.K., Nelson R.S.:

Natural history study of gallbladder cancer.

Cancer 42: 330-335, 1978.

276 Pertsemlidis D., Panveliwalla D., Ahrens E.H. jr.:

Effects of clofibrate and of an estrogen-progestin combination on fasting biliary lipids and cholic acid kinetics in man.

Gastroenterology 66: 565-573, 1974.

277 Piehler J.M., Crichlow R.W::

Primary carcinoma of the gallbladder.

Surg. Gyn. Obst. 147: 929-942, 1978.

278 Pitt H.A., Postier R.G., Cameron J.C.:

Postoperative T-tube cholangiography; Is antibiotic coverage necessary?

Ann. Surg. 191:30-34, 1980.

279 Ponz de Leon M., Murphy G.M., Dowling R.H.:

Physiologic factors influencing serum bile acid levels.

Gut 19:32-39, 1978.

280 Popiela T., Karcz D., Kulig J.:

Significance of intraoperative fibercholangioscopy in the diagnosis of biliary tract disorders.

Endoscopy 10: 275-278, 1978.

281 Prasad J.K.:

A comparison of the value of measurements of flow and pressure as aids to bile-duct surgery.

Br. J. Surg. 58: 868, 1971.

282 Pridgen J.E., Bradley Aust. J., McInnis W.D.:

Primary intrahepatic gallstones.

Arch. Surg. 112: 1037-1044, 1977.

283 Putz P., Willems G.:

Proliferative changes in the epithelium of the human lithiasic galibladder.

J. Nat. Canc. Inst. 60: 283-287, 1978.

284 Udod V.M.. Pleskacheva L.A.:

Clinical significance of hepatorheographic indices in patients with cholelithiasis.

Terap. Arkh. 616: 52-55, 1972. 
285 Urakami Y., Kishi S:

Endoscopic fistulotomy (EFT) for parapapillary choledochoduodenal fistula Endoscopy 10: 289-294, 1978 .

286 Ranson J.H.C.:

The timing of biliary surgery in acute pancreatitis.

Ann. Surg. 189:654-663, 1979.

287 Ranson J.H.C.:

Acute pancreatitis.

Curr. Pr. Surg. XVI no II. 1979.

288 Redinger R.N.:

Pathophysiology of cholelithiasis.

Schweiz. Med. W/sch. 104: 1673-1679, 1974.

289 Redinger R.N.:

The effect of non-functioning gallbiadders in bile composition.

Clin. Res. 22: 367A, 1974.

290 Redinger R.N.:

The effect of loss of gallbladder function on biliary lipid composition in subject with cholesterol gallstones.

Gastroenterology 71: 470-474, 1976.

291 Redinger R.N., Small D.M.:

Bile composition, bile salt metabolism and gallstones.

Arch. Intern. Med. 130:618-630,1972.

292 Redinger R.N., Strasberg S.M., Small D.M.

Primate biliary physiology. IX. Effects of acute biliary obstruction on billary lipid metabolism in the monkey.

Am. J. Phys. 226: 776-783, 1974.

293 Reddy B.S. Wynder E.L.:

Metabolic epidemiology of colon cancer. Fecal bile acids and neutral sterols in colon cancer patients and patients with adenomatous polyps.

Cancer 39:2533-2539, 1977 .

294 Reichelt H.G., Pichlmayr R.

Nuklearmedizinisch-diagnostische Exploration Biliodigestiver Anastomosen.

Fortschr. Röntgenstr. 127:567-571, 1977.

295 Reiter J.J., Bayer H.P., Mennicken C., Manegold B.C.:

Results of endoscopy papillotomy; a collective experience from nine endoscopic centers in West Germany.

World J. Surg. 2: 505-511, 1978.

296 Riemann J.F., Koch H.:

Endoscopy of the billiary tract and the pancreas in children.

Endoscopy 10: 166-172, 1978.

297 Rives J., Lardennois B., Flament J.B:

A modern operating room for radio-surgery of the digestive system.

Med. Chir. Dig. 6:31-38, 1977. 
298 Roda E., Aldini R., Mazzella G., Roda A.

Enterohepatic circulation of bile acids after cholecystectomy.

Gut 19:640-649, 1978.

299 Rösch W., Koch H.:

Peroral cholangioscopy in choledocho-duodenostomy-patients using the pediatric fiberscope.

Endoscopy 10: 195-198, 1978.

300 Rose A.G.

Primary sarcoma of the gallbladder. A case report.

S. Afr. Med. J. 53: 909-910, 1978.

301 Roslyn J.J., DenBesten L., Thompson J.E., Cohen K.:

Chronic cholelithiasis and decreased bile salt pool size: cause or effect?

Am. J. Surg. 139: 119-124, 1980.

302 Rosseland A., Osnes M., Kruse A., Skrede M.:

Endoscopic papillotomy (EPT) with removal of common bile duct stones.

Acta Chir. Scand. 143: 49-52, 1977.

303 Ruys J.H.J., Hulst S.G.Th.:

Diagnosis of cholecystoses. A radiologic survey with clinical aspects.

Martinus Nyhoff, The Hague, 1977.

304 Rutledge R.H.:

Sphincteroplasty and choledochoduodenostomy for benign billiary obstructions.

Ann. Surg. 183: 476-487, 1976.

305 Safrany L.:

Transduodenal endoscopic sphincterotomy and extraction of bile duct stones.

World J. Surg. 2: 457-464, 1978.

306 Saharia P.C., Zuidema G.D., Cameron J.L.:

Primary common duct stones.

Am. J. Surgery 185: 595-604, 1977.

307 Sarles H., Sahel J.:

Cholestasis and lesions of the biliary tract in chronic pancreatitis.

Gut 19:851-857.1978.

308 Salmenkivi K:

Cholesterolosis of the gallibladder.

Acta Chir. Scand. suppl. 324, 1964.

309 Salomon J., Roseman D.L.:

Intraoperative measurement of common duct resistance.

Arch. Surg. 113: 650-652, 1978.

310 Sapala M.A. Steel W.B., Resto Soto A.D., Sapala J.A.:

Ultrasonic scanning in post-cholecystectomy choledocholithiasis.

Surgery 82: 420-424, 1977.

311 Sarles H., Sahel J.:

Progress report. Cholestasis and lesions of the biliary tract in chronic pancreatitis.

Gut 19:851-857, 1978. 
312 Schein Cl.1:

Acute cholecystitis.

Harper \& Row, New York 1972.

313 schein Cl.J:

Choledochal incontinence as a purposeful adjunct to choledocholithotomy.

Surg. Gyn. Obst. 144: 157, 1977.

314 Schein $C$. . Beneventano T.C.:

Biliary manometry: its role in clinical surgery.

Surgery $67: 255-260,1970$.

315 Schein Cl.J., Beneventano T.C, Rosen R.G., Dardik H.M.:

Hepatic plexus vagectomy as an adjunct to cholecystectomy.

Surg. Gyn. Obst. 128:241-251, 1969.

316 Schein CI.J., El Tawil V., Dardik H., Beneventano T.C.:

Common duct dynamics in man.

Am. J. Surg. 119:261-263,1970.

317 Schein C... Gliedman M.K.

Choledochoduodenostomy as an adjunct to choledocholithotomy.

Surg. Gyn. Obst. 146:25-27, 1978.

318 Scheín Cl.J., Mahadevia P':

Surgical significance of the histopathology of the common bile duct.

Am. J. Surg. 137: 763-767.1979.

319 Schein Cl.J. Stern W.Z., Hurwitt E.S., Jacobson H.G.:

Cholangiography and biliary endoscopy as complementary methods of

evaluating the bile ducts.

Surgery 53: 864-875, 1963.

320 Schein $\mathrm{Cl} . J$., Stern W. Z. Jacabson H.G.:

The hepatic ductal system: a correlation of endoscopic and roentgenograhic findings.

Surgery $51: 718-732,1962$.

321 Schersten T:

Formation of lithogenic bile in man.

Digestion 9: $540-553,1973$.

322 Schmidt W.J.H.:

Percutane transperitoneale transhepatische cholangiografie.

Ziekten der Galwegen, Lunteren 1974.

323 Schmitt J.C. Sava G.:

Operative trauma to the common bile duct: a report of a series of 12 cases.

Chirurgie 103: 216-225, 1977.

324 Schoenfield L.J.:

(Editorials): The disappearing gallstone and the national cooperative gallstone study.

JAMA 239:1162, 1978.

325 Schuiman $A$ :

Biliary ascariasis presenting in the United States.

Am. J. Gastroenterology 68: 167-170, 1977. 


\section{Schwarz D.:}

Die Komplikationen der Cholelithiasis. Zschr. Ärtzl. Fortbild. 66: 75-79, 1971.

327 Scott A.J.:

Are there proliferative compartments in the gallbladder? Gastroenterology 67: 1231-1237, 1974.

328 Scott Jones R., Smith B.M.:

The effect of truncal vagotomy on taurocholate choleresis.

J. Surg. Res. 23: 149-154, 1977.

329 Seif R.M.:

Routine operative cholangiography: A critial appraisal.

Am. J. Surg. 134:566-568, 1977.

330 Selle J.G., Altemeier W/A., Fullen W.D., Goldsmith R.E.:

Cholelithiasis in hyperparathyroidism.

Arch. Surg. 105: 369-374, 1972.

331 Shaffer E.A., Braash J.W., Small D.M.:

Bile composition at and after surgery in normal persons and patients with gallstones.

N. Eng. J. Med. 287: 1317-1322, 1972.

332 Shaw R.A., Jones R.S.:

The choleretic action of cholecystokinin and cholecystokinin octapeptide in dogs.

Surgery 85: 622-625, 1978 .

333 Shields $H$.:

Occurrence of an adenocarcinoma at the choledochoenteric anastomosis 14 years after pancreatoduodenectomy for benign disease.

Gastroenterology 72: 322-324, 1977.

334 Shiu Kum Lam., Kai Ping Wong., Chan P.K.W., Ngan H.

Recurrent pyogenic cholangitis: a study by endoscopic retrograde cholangiography.

Gastroenterology 74: 1196-1203, 1978.

335 Shore J.M., Berci G., Morgenstern L.:

The value of biliary endoscopy.

Surg. Gyn. Obst. 140: 601-604, 1975.

336 Shore J.M., Berci G.

An improved flexible cholangioscope.

Endoscopy 8:41-42, 1976.

337 Shore J.M.r Lippman H.N.:

A flexible choledochoscope.

Lancet I: 1200-1201, 1965.

338 Shore J.M. . Morgenstern L., Berci G.:

An improved rigid choledochoscope.

Am. J. Surg. 122: 567-568, 1971. 
339 Shore J.M., Morgenstern L., Hamlin J.A.

Choledochoscopy and operative fluorocholangiography in the prevention of retained bile duct stones.

World J. Surg. 2: 411-427, 1978.

340 Siegel J.H.:

Endoscopic Papillotomy: Sphincterotomy or Sphincterolplasty.

Am. J. Gastroenterology 72:511-516, 1979.

341 sier J.C.:

The influence of truncal vagotomy on the composition of bile.

Thesis, Vrije Universiteit, Amsterdam 1975.

342 Silen W., Wertheimer $M$. Kirschenbaum $G$ :

Bacterial contamination of the biliary tree after choledochostomy.

Am. J. Surg. 135: 325-327, 1978.

343 Sivennoien $E$.:

Concrements resulting from suture material in the billiary tract. A clinical and experimental study.

Thesis, Helsinki 1970.

344 Silvis S.E.

A Reevaluation of chalangiography.

Am. J. Dig. Dis. 23: 577-578, 1978.

345 Simi M., Loriga P., Basoli A., Leardi S.:

Intrahepatic lithiasis. Study of thirty-six cases and review of the literature.

Am. J. Surg. 137: 317-322, 1979.

346 Simmons F., Ross A.P.J., Bouchier I.A.D.:

Alterations in hepatic bile composition after cholecystectomy.

Gastroenterology 63: 466-471, 1972.

347 Skillings J.C., Williams IS. Hinshaw J.:

Cost-effectiveness of operative cholangiography.

A.m. J. Surg. 137:26-31, 1979.

348 Slooff M., Baher R.:

What is involved in endoscopic sphincterotomy for gallstones?

Br. J. Surg. 67: 18-21, 1980.

349 Smith D.C., McAllister R.A., Mackay C.:

Proceedings: The effect of cholecystectomy on the composition of bile in gallstone patients.

Br. J. Surg. 60: 899, 1977.

350 Smith 5.W. Engel Ch., Averbook B., Longmire jr. W.P.:

Problems of retained and recurrent common bile duct stones.

JAMA 164: 321-235, 1957.

351 Smith III R.B., Conklin F., Porter M.R.:

A five year study of choledocholithiasis.

Surg. Gyn. Obst. 116:731-740,1963.

352 Smoron G.L.:

Radiation therapy of carcinoma of gallbladder and biliary tract.

Cancer 40: 1422-1424, 1977. 
353 Snow J.R., Scott Jones R:

The effect of insulin on bile-salt-independent canalicular secretion.

Surgery 83: 458-463, 1978.

354 Sobol S., Cooperman A.M.:

Villous adenoma of the ampulla of Vater. An unusual cause of biliary colic and obstructive jaundice.

Gastroenterology 75: 107-109,1978.

355 Spitaels J.M., Lloyd D.A., Boultbee J.E.:

Biliary ascariasils.

S. Afr. Med. J. 54: 1044, 1978.

356 Spuy S. v.d.:

Endoscopic retrograde cholangio-pancreatography (ERCP) in children.

Endoscopy 10: 173-175, 1978.

357 Stein $T$., Wise L.:

Biliary lipid composition after cholecystectomy.

Surg. Res. 18: 505-511, 1975.

358 Stephens C.G., Scott R.B.:

Cholelithiasis in Sickle Cell Anemia.

Arch. Intern. Med. 140:648-651, 1980.

359 Stiehl A., Czygan P., Kommerell B., Weis H.J.:

Ursodeoxycholic acid versus chenodeoxycholic acid. Comparison of their effects on bile acid and bile lipid composition in patients with cholesterol gallstones.

Gastroenterology 75: 1016-1021, 1978.

360 Stotter L., Wiendl H.J., Ultsch B.:

An improved flexible cholangioscope.

Endoscopy 7: 160-163, 1975.

361 Stout R.W., Balmer J.P., Henry R.W., Buchanan K.D.:

Plasma lipids and gastro-intestinal hormones in subjects with gallstones.

Horm. Metab. Res. 10: 357-358, 1978.

362 Strasberg S.M., Fisher M.M.:

Pathogenesis of human cholesterol cholelithiasis.

Can. Med. Ass. J. 112: 484-488, 1975.

363 Stuart M. Hoerr S.O.:

Late results of side to side choledochoduodenostomy and of transduodenal sphincterotomy for benign disorder. A twenty year comparative study.

Am. J. Surg. 123:67-72, 1972.

364 Sturdevant R.A.L., Pearce M.L., Dayton S.:

Increased prevallence of cholelithiasis in men ingesting a serum-cholesterollowering diet.

N. Eng. J. Med. 288: 24-27, 1973.

365 Sugrue W.J.:

Operative cholangiography in two hundred consecutive cholecystectomies.

N.Z. Med. J. 86:470-471, 1977. 
366 Sutor J.J., Wooley S.E:

The sequental deposition of crystalline material in gallstones: evidence for changing gallbladder bile composition during the growth of some stones.

Gut 15:130-131, 1974 .

367 Svane S., Wetteland P.:

Obstructive blood thrombus formation in the common bile duct probably due to a silk ligature.

Acta Chir. Scand. 144:553-556, 1978.

368 Swell L.:

The cholesterol Saturation Index of Human bile.

Am. 1. Dis. 19:261-265, 1974.

369 Tangedahl T.:

Dissolution of gallstones. When and How?

Surg. Cl. North Am. 59:797-809, 1979.

370 Taub M., Coyne M.J., Bonorris G.G., Schoenfield L.J.:

Inhibition by propranolol of bile acid-and PGE-stimulated CAMP and intestinal secretion.

Am. J. Gastroenterology 70: 129-135, 1978.

37 1 Taylor White T., Bordley IV J:

One per cent incidence of recurrent gallstones six to eight years after

manometric cholangiography.

Ann. Surg. 188: 562-569, 1978.

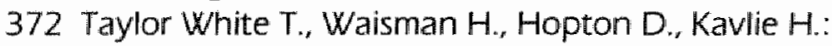

Radiomenometry, flow rates, and cholangiography in the evaluation of common bile duct disease.

Am. J. Surg. 123:73-79, 1980.

373 Tchirkow G., Highman L.M. Shafer A.D.:

Cholelithiasis and Cholecystitis in children after repair of congenital duodenal

Anomalles.

Arch. Surg. 155:85-86, 1980.

374 Tehirkow G., Silver S.C.:

Injury to the portal vein. A hazard during common bile duct exploration.

Arch. Surg. 113:745-747, 1978.

375 Thistle J.L., Carison G.L., Hofmann A.F.:

Monooctanoin, a dissolution agent for retained cholesterol bile duct stones.

Gastroenterology 78: 1016-1022, 1980.

376 Thistle J.L., Hofmann A.F., Ott B.J., Stevens D.H.:

Chenotherapy for gallstones dissolution.

JAMA 239: 1041-1046, 1978.

377 Thistle J.L., Yu P.Y.S., Hofmann A.F. and Ott B.J.:

Prompt return of bile to supersaturated state followed by gallstone recurrence after discontinuance of chenodeoxycholic acid therapy.

Gastroenterology 66: A-135/789, 1974. 
378 Thurston O.G:

Dynamics of cholesterol gallstone dissolution in canin bile.

J. Surg. Res. 24:215-219. 1978.

379 Thurston O.G., MCDougall R.M.:

The effect of hepatic bile on retained common duct stones.

Surg. Gyn. Obst. 143: 625-627, 1976.

380 Thurston O.G., MCDougall R.M., Walker K.:

The effect of prosthetic gallstones on totall bile acid pool size in dogs.

Am. J. Surg. 139: 237-239, 1980.

381 Tompkins R.K., Croke J.C:

Bile Acid Inhibit Pepsin Activity Against a Refined Substrate.

Am. J. Surg. 139, 237-239, 1980.

382 Tompkins R.K., Jonnson J., Storm F.K., Longmire W.P.:

Operative endoscopy in the management of biliary tract neoplasms.

Am. J. Surg. 32: 174-182, 1976.

383 Toouli J., Williamson B.W/A., Gooszen H., Blumgart L.H.:

in vitro dissolution of human gallstones; the efficacy of heparinized solutions.

Br. J. Surg. 66:770-771, 1979.

384 Towne J.B., Bernhard V.M.:

Vascular endoscopy: useful tool or interesting toy.

Surgery 82: 415-419, 1977.

385 Towne J.B., Bernhard V.M.:

Technique of intraoperative endoscopic evaluation of occluded aortofemoral grafts following thrombectomy.

Surg. Gyn. Obst. 148: 87-89, 1979.

386 Tran Ky:

Choledochoduodenostomy versus choledochojejunostomy.

Surgery 83: 366, 1978.

387 Treadwell T.A., Hardin W.J.:

Primary carcinoma of the gallbladder. The role of adjunctive therapy in its treatment.

Am. J. Surg. 132: 703-706, 1976.

388 Tseng A., Sales D.J., Simonowitz D.A., Enker W.E.

Pancreatic abcess: a fatal complication of endoscopic cholangiopancreatography

(ERCP).

Endoscopy 9: 250-253, 1977.

389 Tsuzuki T., Uekasa M.:

Carcinoma of the proximal bile ducts.

Surg. Gyn. Obst. 146: 933-938, 1978.

390 Valdevieso V., Palma R., W/unkhaus R., Antezana C.:

Effect of aging on biliary lipid composition and bile acid metabolism in normal

Chilean women.

Gastroenterology 74:871-874, 1978. 
391 Vellacott K.D., Powell P.H.:

Exploration of the common bille duct: a comparative study.

Br. J. Surg. 66:389-391, 1979.

392 Vergroesen A.J:

Nieuwe aspecten van de fysiologische betekenis van linolzuur.

Excerpta Medica 1978.

393 Vernick, L.J, Kuller L.H.:

Relationship between cholecystectomy and Ascending Colon Cancer.

Cancer $45: 392-395,1980$.

393a Vernick L.J, Kuller L.H.;

Cholecystectomy and richt-sided colon cancer: an epidemiological study.

Lancet 11: $381-383.1981$

394 Vogt D.P., Hermann R.E.

Choledochoduodenostomy, Choledochojejunostomy or Sphincteroplasty for

Biliary and Pancreatic Disease.

Ann. Surg. 193: 161-168, 1981.

395 Vollmar J., Storz L.W., Martins de Souza Torres J.:

Gefässendoskopie, Möglichkeiten und Grenzen ihrer Anwendbarkeit.

Chirurg 44:222-227, 1973.

396 Wählby L.:

Aplasia of the gallbladder with common duct stones.

Acta Chir. Scan. 143: 241-243, 1977.

397 Wall C.A., Peartree S.P.:

Practical value of operative cholangiography.

JAMA 164: 236-238, 1957.

398 W/all J.W.M. v.d.:

Cholelithiasis, symptoom of diagnose?

N.T.V.G. 116:1282-1288, 1972.

399 Walland D.R.

in vitro studies of gallstone dissolution using bile salt solutions and heparinized saline.

Br. J. Surg. 64: 572-576, 1977 .

400 Wani N.A., Kala Z.5., Rashid P.A.:

Postvagotomy cholelithiasis.

Int. Surg. 62: $481-482,1977$.

401 Warshaw A.L:

Bile Gastritis without prior gastric surgery: contributing role of cholecystectomy.

Am. J. Surg. 137: 527-531, 1979.

402 Warshaw L., Bartlett M.K...

Technic for finding and removing stones from intrahepatic bile ducts.

Am.J. Surg. 127:353-354, 1974. 
403 Watkin D.F.I., Thomas G.G.:

Jaundice in acute cholecystitis.

Br. J. Surg. 58:570-573, 1971.

404 Watts J.MCK, Jablonski P., Toouli J.

The effect of added bran to the diet on the saturation of bile in people without gallstones.

Am. J. Surg. 135: 321-324, 1978.

405 Way L.W:

Retained common duct stones.

Surg. Cl. North Am. 53: 1139-1147, 1973.

406 Way L.W., Admirand W.H., Dunphy J.E.:

Management of choledocholithiasis.

Ann. Surg. 176: 347-359, 1972.

407 Weel M.W.V.:

Het directe risico van cholecystectomie: een analyse van 1458 operaties.

N.T.V.G. 103: 6-11, 1959.

408 Weel M.W. v.:

Indicaties tot operatieve behandeling bij steenlijden en ontsteking.

Ziekten van de galwegen, Symposion Lunteren 1974.

409 Weithofer G., Blazek Z., Warm K., Bloch R.:

Spontaneous expulsion of a migrating infantry missile impacted in the duodenum and the common bile duct, 32 years after wounding.

Endoscopy 9: 106-109, 1977.

410 Wenckert A., Robertson B.:

The natural course of gallstone disease. Eleven-year review of 781

nonoperated cases.

Gastroenterology 50: 376-381, 1966.

411 Wheeler H.O.:

Pathogenesis of gallstones.

Surg. Cl. North Am. 53: 963-972, 1973.

412 Wiechel K-U.:

Percuteaneous Transhepatic Cholangiography.

Acta Chir. Scan. sup 330, 1964

413 Wildegans $\mathrm{H}$ :

Die operatieve Gallengangendoskopie, p. 80 .

Urban \& Schwarzenberg, W/ien, 1960.

414 Wildegans $\mathrm{H}$.:

Die Endoskopie der Gallengange.

Dtsch. Med. W/schr. 40: 1775-1779, 1958.

415 Wilhelm A.

Endoskopie der Gallenwege.

Chir. Praxis 22: 227-238, 1977.

416 Williams E.J., Irvine W.T.

Functional and metabolic effects of total and selective vagotomy.

Lancet II : 1053-1057, "966. 
417 Williams R.C., Showalter R., Kern F:

In vivo effect of bile salts and cholestyramine on intestinal anaerobic bacteria.

Gastroenterology 69, 483-491, 1975.

418 Williams R.D. Huang T.T.:

The effect of vagotomy on biliary pressure.

Surgery $66: 353-356,1969$.

419 Wojtowicz J., Karwowski A.

Cholangiographic appearance of the intrahepatic bile ducts.

Fortschr, Rontgenstr. 127:417-421, 1977.

420 Wollman M.D., Greenhow D.E.:

Recent development in anesthesia.

Surg. Cl. North. Am. 55: 787794, 1975.

421 Wolloch Y., Feigenberg Z., Zer M., Dintsman M.

The influence of biliary infection on the postoperative course after biliary tract surgery.

Am. J. Gastroenterology 67: 456-462, 1977.

422 Wood M.:

Eponyms in biliary tract surgery.

Am. J. Surg. 138: 746-754, 1979.

423 Yamaguchi M:

Congenital Choledochal Cyst.

Am. J. Surg. 140:653-656, 1980.

424 Zimmon D.S.:

Endoscopic diagnosis and management of biliary and pancreatic disease.

Curr. Pr. Surg. 26: 1-42, 1979.

425 Zunkel D.E.:

Physiologic observations of the gallbladder in pregnancy.

Non-invasive radiodiagnosis; Boerhaave course 1980, Leiden. 


\section{Curriculum Vitae}

The author was born on February 28,1949 in The Hague, Netherlands.

He graduated from High School "Overvoorde" in The Hague in 1967 and attended the Medical Faculty at the Free University in Amsterdam.

He received his Medical Degree in 1973.

In 1974 he worked in the St. Elisabeth Ziekenhuis in Alkmaar at the surgical department of Piet van Velthoven, M.D. C.S.

In 1975 he joined the Military Medical Corps.

In Jully 1976 he started his residency at the surgical department of the

De W/ever Hospital in Heerlen under supervision of Jaap Munting, M.D. Ph.D..

His registration in general surgery will take place in July 1982. 